PACS: $02.20 . \mathrm{Bb}, 02.20 . \mathrm{Uw}, 02.10 . \mathrm{Hh}$

\title{
POLYADIC HOPF ALGEBRAS AND QUANTUM GROUPS
}

\author{
Steven Duplij \\ WWU IT, Universität Münster, D-48149 Münster, Germany \\ E-mail:douplii@wwu.de,https://ivv5hpp.uni-muenster.de/u/douplii
}

Received March 8, 2021; accepted May 8, 2021

This article continues the study of concrete algebra-like structures in our polyadic approach, where the arities of all operations are initially taken as arbitrary, but the relations between them, the arity shapes, are to be found from some natural conditions ("arity freedom principle"). In this way, generalized associative algebras, coassociative coalgebras, bialgebras and Hopf algebras are defined and investigated. They have many unusual features in comparison with the binary case. For instance, both the algebra and its underlying field can be zeroless and nonunital, the existence of the unit and counit is not obligatory, and the dimension of the algebra is not arbitrary, but "quantized". The polyadic convolution product and bialgebra can be defined, and when the algebra and coalgebra have unequal arities, the polyadic version of the antipode, the querantipode, has different properties. As a possible application to quantum group theory, we introduce the polyadic version of braidings, almost co-commutativity, quasitriangularity and the equations for the $R$-matrix (which can be treated as a polyadic analog of the Yang-Baxter equation). We propose another concept of deformation which is governed not by the twist map, but by the medial map, where only the latter is unique in the polyadic case. We present the corresponding braidings, almost co-mediality and $M$-matrix, for which the compatibility equations are found.

KEY WORDS: polyadic field, polyadic algebra, bialgebra, Hopf algebra, antipode, braid equation, braiding, R-matrix, Yang-Baxter equation, mediality, co-medaility, M-matrix, quasitriangularity

\section{INTRODUCTION}

Since Hopf algebras were introduced in connection with algebraic topology [1,2], their role has increased significantly (see, e.g., [3]), with numerous applications in diverse areas, especially in relation to quantum groups [4-8]. There have been many generalizations of Hopf algebras (for a brief review, see, e.g., [9]).

From another perspective, the concepts of polyadic vector space, polyadic algebras and polyadic tensor product over general polyadic fields were introduced in [10]. They differ from the standard definitions of $n$-ary algebras [11-13] in considering an arbitrary arity shape for all operations, and not the algebra multiplication alone. This means that the arities of addition in the algebra, the multiplication and addition in the underlying field can all be different from binary and the number of places in the multiaction (polyadic module) can be more than one [14]. The connection between arities is determined by their arity shapes [10] ("arity freedom principle"). Note that our approach is somewhat different from the operad approach (see, e.g., $[15,16]$ ).

Here we propose a similar and consequent polyadic generalization of Hopf algebras. First, we define polyadic coalgebras and study their homomorphisms and tensor products. In the construction of the polyadic convolution product and bialgebras we propose considering different arities for the algebra and coalgebra, which is a crucial difference from the binary case. Instead of the antipode, we introduce its polyadic version, the querantipode, by analogy with the querelement in $n$-ary groups [17]. We then consider polyadic analogs of braidings, almost co-commutativity and the $R$-matrix, together with the quasitriangularity equations. This description is not unique, as with the polyadic analog of the twist map, while the medial map is unique for all arities. Therefore, a new (unique) concept of deformation is proposed: almost co-mediality with the corresponding $M$-matrix. The medial analogs of braidings and quasitriangularity are introduced, and the equations for $M$-matrix are obtained.

\section{POLYADIC FIELDS AND VECTOR SPACES}

Let $\mathbb{k}=\mathbb{k}^{\left(m_{k}, n_{k}\right)}=\left\langle K \mid \nu_{k}^{\left(m_{k}\right)}, \mu_{k}^{\left(n_{k}\right)}\right\rangle$ be a polyadic or $\left(m_{k}, n_{k}\right)$-ary field with $n_{k}$-ary multiplication $\mu_{k}^{\left(n_{k}\right)}$ : $K^{n_{k}} \rightarrow K$ and $m_{k}$-ary addition $\nu_{k}^{\left(m_{k}\right)}: K^{m_{k}} \rightarrow K$ which are (polyadically) associative and distributive, such that $\left\langle K \mid \mu^{\left(n_{k}\right)}\right\rangle$ and $\left\langle K \mid \nu^{\left(m_{k}\right)}\right\rangle$ are both commutative polyadic groups [18,19]. This means that $\mu_{k}^{\left(n_{k}\right)}=\mu_{k}^{\left(n_{k}\right)} \circ \tau_{n_{k}}$ and $\nu_{k}^{\left(m_{k}\right)}=\nu_{k}^{\left(m_{k}\right)} \circ \tau_{m_{k}}$, where $\tau_{n_{k}} \in S_{n_{k}}, \tau_{m_{k}} \in S_{m_{k}}$, and $S_{n_{k}}, S_{m_{k}}$ are the symmetry permutation groups. A polyadic field $\mathbb{k}^{\left(m_{k}, n_{k}\right)}$ is derived, if $\mu_{k}^{\left(n_{k}\right)}$ and $\nu_{k}^{\left(m_{k}\right)}$ are iterations of the corresponding binary operations: ordinary multiplication and addition. The polyadic fields considered in [19] were derived. The simplest example of a nonderived $(2,3)$-ary field is $\mathbb{k}^{(2,3)}=\{i \mathbb{R}\}$, and of a nonderived $(3,3)$-ary field is $\mathbb{k}^{(3,3)}=\{i p / q\}$, where $p, q \in \mathbb{Z}^{\text {odd }}\left(i^{2}=-1\right.$, and the operations are in $\left.\mathbb{C}\right)$. Polyadic analogs of prime Galois fields including nonderived ones were presented in [20]. 
Recall that a polyadic zero $z$ in any $\left\langle X \mid \nu^{(m)}\right\rangle$ (with $\nu^{(m)}$ being an addition-like operation) is defined (if it exists) by

$$
\nu^{(m)}[\hat{\boldsymbol{x}}, z]=z, \quad \forall \hat{\boldsymbol{x}} \in X^{m-1},
$$

where $z$ can be on any place, and $\boldsymbol{x}$ is any polyad of length $m-1$ (as a sequence of elements) in $X$. A polyadic unit in any $\left\langle X \mid \mu^{(n)}\right\rangle$ (with $\mu$ being a multiplication-like operation) is an $e \in X$ (if it exists) such that

$$
\mu^{(n)}\left[e^{n-1}, x\right]=x, \quad \forall x \in X,
$$

where $x$ can be on any place, and the repeated entries in a polyad are denoted by a power $\overbrace{x, \ldots, x}^{n} \equiv x^{n}$. It follows from (2), that for $n \geq 3$ the polyad $\boldsymbol{e}$ can play the role of a unit, and is called a neutral sequence [21]

$$
\mu^{(n)}[\hat{\boldsymbol{e}}, x]=x, \quad \forall x \in X, \quad \hat{\boldsymbol{e}} \in X^{n-1} .
$$

This is a crucial difference from the binary case, as the neutral sequence $\hat{\boldsymbol{e}}$ can (possibly) be nonunique.

The nonderived polyadic fields obey unusual properties: they can have several (polyadic) units or no units at all (nonunital, as in $\mathbb{k}^{(2,3)}$ and $\mathbb{k}^{(3,3)}$ above), no (polyadic) zeros (zeroless, as $\mathbb{k}^{(3,3)}$ above), or they can consist of units only (for some examples, see [20,22]). This may lead, in general, to new features of the algebraic structures using the polyadic fields as the underlying fields (e.g. scalars for vector spaces, etc.) [10].

Moreover, polyadic invertibility is not connected with units, but is governed by the special element, analogous to an inverse, the so called querelement $\bar{x}$, which for any $\left\langle X \mid \mu^{(n)}\right\rangle$ is defined by [17]

$$
\mu^{(n)}\left[x^{n-1}, \bar{x}\right]=x, \quad \forall x \in X,
$$

where $\bar{x}$ can be on any place (instead of the binary inverse " $x x^{-1}=e$ "). An element $x \in X$ for which (4) has a solution under $\bar{x}$ is called querable or "polyadically invertible". If all elements in $X$ are querable, and the operation $\mu^{(n)}$ is polyadically associative, then $\left\langle X \mid \mu^{(n)}\right\rangle$ is a $n$-ary group. Polyadic associativity in $\left\langle X \mid \mu^{(n)}\right\rangle$ can be defined as a kind of invariance relationship [14]

$$
\mu^{(n)}\left[\hat{\boldsymbol{x}}, \mu^{(n)}[\hat{\boldsymbol{y}}], \hat{\boldsymbol{z}}\right]=\text { invariant },
$$

where $\hat{\boldsymbol{x}}, \hat{\boldsymbol{y}}, \hat{\boldsymbol{z}}$ are polyads of the needed size in $X$, and $\mu^{(n)}[\hat{\boldsymbol{y}}]$ can be on any place, and we therefore will not use additional brackets. Using polyadic associativity (5) we introduce $\ell$-iterated multiplication by

$$
\left(\mu^{(n)}\right)^{\circ \ell}[\hat{\boldsymbol{x}}]=\overbrace{\mu^{(n)}\left[\mu ^ { ( n ) } \left[\ldots \mu^{(n)}\right.\right.}^{\ell}[\hat{\boldsymbol{x}}]]], \quad \hat{\boldsymbol{x}} \in X^{\ell(n-1)+1},
$$

where $\ell$ is "number of multiplications". Therefore, the admissible length of any $n$-ary word is not arbitrary, as in the binary $n=2$ case, but fixed ("quantized") to $\ell(n-1)+1$.

Example 1. Consider the nonunital zeroless polyadic field $\mathbb{k}^{(3,3)}=\{i p / q\}, i^{2}=-1, p, q \in \mathbb{Z}^{\text {odd }}$ (from the example above). Both the ternary addition $\nu^{(3)}[x, y, t]=x+y+t$ and the ternary multiplication $\mu^{(3)}[x, y, t]=x y t$ are nonderived, ternary associative and distributive. For each $x=i p / q\left(p, q \in \mathbb{Z}^{\text {odd }}\right)$ the additive querelement (denoted by a wave, a ternary analog of an inverse element with respect to addition) is $\tilde{x}=-i p / p^{\prime}$, and the multiplicative querelement is $\bar{x}=-i q / p$ (see (4)). Therefore, both $\left\langle\{i p / q\} \mid \mu^{(3)}\right\rangle$ and $\left\langle\{i p / q\} \mid \nu^{(3)}\right\rangle$ are ternary groups (as it should be for a (3,3)-field), but they contain no neutral elements (unit or zero).

The polyadic analogs of vector spaces and tensor products were introduced in [10]. Briefly, consider a set $V$ of "polyadic vectors" with the addition-like $m_{v}$-ary operation $\nu_{V}^{\left(m_{v}\right)}$, such that $\left\langle V \mid \nu_{v}^{\left(m_{v}\right)}\right\rangle$ is a commutative $m_{v}$-ary group. The key differences from the binary case are: 1$)$ The zero vector $z_{v}$ does not necessarily exist (see the above example for $\mathbb{k}^{(3,3)}$ field); 2) The role of a negative vector is played by the additive querelement $\tilde{v}$ in $\left\langle V \mid \nu_{V}^{\left(m_{v}\right)}\right\rangle$ (which does not imply the existence of $z_{v}$ ). A polyadic analog of the binary multiplication by a scalar $(\lambda v)$ is the multiaction $\rho^{\left(r_{\rho}\right)}$ introduced in [14]

$$
\rho_{V}^{\left(r_{v}\right)}: K^{r_{v}} \times V \rightarrow V .
$$

If the unit $e_{k}$ exists in $\mathbb{k}^{\left(m_{k}, n_{k}\right)}$, then the multiaction can be normalized (analog of " $1 v=v$ ") by

$$
\rho_{V}^{\left(r_{v}\right)}\left(e_{k}^{\times r_{v}} \mid v\right)=v, \quad v \in V
$$


Under the composition $\circ_{n_{\rho}}$ (given by the arity changing formula [14]), the set of multiactions form a $n_{\rho}$-ary semigroup $S_{\rho}^{\left(n_{\rho}\right)}=\left\langle\left\{\rho_{V}^{\left(r_{v}\right)}\right\} \mid \circ_{n_{\rho}}\right\rangle$. Its arity is less or equal than $n_{k}$ and depends on one integer parameter (the number of intact elements in the composition), which is less than $\left(r_{v}-1\right)$ (for details see [10]).

A polyadic vector space over the polyadic field $\mathbb{k}^{\left(m_{k}, n_{k}\right)}$ is

$$
V=V^{\left(m_{v} ; m_{k}, n_{k} ; r_{\rho}\right)}=\left\langle V, K \mid \nu_{V}^{\left(m_{v}\right)} ; \nu_{k}^{\left(m_{k}\right)}, \mu_{k}^{\left(n_{k}\right)} ; \rho_{V}^{\left(r_{v}\right)}\right\rangle
$$

where $\left\langle V \mid \nu_{V}^{\left(m_{v}\right)}\right\rangle$ is a commutative $m_{v}$-ary group, $\left\langle K \mid \mu_{k}^{\left(n_{k}\right)}, \nu_{k}^{\left(m_{k}\right)}\right\rangle$ is a polyadic field, $\left\langle\left\{\rho_{V}^{\left(r_{v}\right)}\right\} \mid \circ_{n_{\rho}}\right\rangle$ is a $n_{\rho}$-ary semigroup, the multiaction $\rho^{\left(r_{\rho}\right)}$ is distributive with respect to the polyadic additions $\nu_{V}^{\left(m_{v}\right)}, \nu_{k}^{\left(m_{k}\right)}$ and compatible with $\mu_{k}^{\left(n_{k}\right)}$ (see (2.15), (2.16), and (2.9) in [10]). If instead of the underlying field, we consider a ring, then (9) define a polyadic module together with (7). The dimension $d_{v}$ of a polyadic vector space is the number of elements in its polyadic basis, and we denote it $V_{d_{v}}=V_{d_{v}}^{\left(m_{v} ; m_{k}, n_{k} ; r_{v}\right)}$. The polyadic direct sum and polyadic tensor product of polyadic vector spaces were constructed in [10] (see (3.25) and (3.39) there). They have an unusual peculiarity (which is not possible in the binary case): the polyadic vector spaces of different arities can be added and multiplied. The polyadic tensor product is "Kk-linear" in the usual sense, only instead of "multiplication by scalar" one uses the multiaction $\rho_{V}^{\left(r_{v}\right)}$ (see [10] for details). Because of associativity, we will use the binary-like notation for polyadic tensor products (implying $\otimes=\otimes_{\mathbb{k}}$ ) and powers of them (for instance, $\overbrace{x \otimes x \otimes \ldots \otimes x}^{n}=x^{\otimes n})$ to be clearer in computations and as customary in diagrams.

\section{POLYADIC ASSOCIATIVE ALGEBRAS}

Here we introduce operations on elements of a polyadic vector space, which leads to the notion of a polyadic algebra.

\section{"Elementwise"description}

Here we formulate the polyadic algebras in terms of sets and operations written in a manifest form. The arities will be initially taken as arbitrary, but then relations between them will follow from compatibility conditions (as in [10]).

Definition 2. A polyadic (associative) algebra (or $\mathbb{k}$-algebra) is a tuple consisting of 2 sets and 5 operations

$$
A=A^{\left(m_{a}, n_{a} ; m_{k}, n_{k} ; r_{a}\right)}=\left\langle A, K \mid \nu_{A}^{\left(m_{a}\right)}, \mu_{A}^{\left(n_{a}\right)} ; \nu_{k}^{\left(m_{k}\right)}, \mu_{k}^{\left(n_{k}\right)} ; \rho_{A}^{\left(r_{a}\right)}\right\rangle
$$

where:

1. $\mathbb{k}^{\left(m_{k}, n_{k}\right)}=\left\langle K \mid \nu_{k}^{\left(m_{k}\right)}, \mu_{k}^{\left(n_{k}\right)}\right\rangle$ is a polyadic field with the $m_{k}$-ary field (scalar) addition $\nu_{k}^{\left(m_{k}\right)}: K^{m_{k}} \rightarrow K$ and $n_{k}$-ary field (scalar) multiplication $\mu_{k}^{\left(n_{k}\right)}: K^{m_{k}} \rightarrow K$;

2 .

$$
A_{\text {vect }}=A^{\left(m_{a} ; m_{k}, n_{k} ; r_{a}\right)}=\left\langle A, K \mid \nu_{A}^{\left(m_{a}\right)} ; \nu_{k}^{\left(m_{k}\right)}, \mu_{k}^{\left(n_{k}\right)} ; \rho_{A}^{\left(r_{a}\right)}\right\rangle
$$

is a polyadic vector space with the $m_{a}$-ary vector addition $\nu_{A}^{\left(m_{a}\right)}: A^{n_{a}} \rightarrow A$ and the $r_{a}$-place multiaction $\rho_{A}^{\left(r_{a}\right)}: K^{r_{a}} \times A \rightarrow A ;$

3. The map $\mu_{A}^{\left(n_{a}\right)}: A^{n_{a}} \rightarrow A$ is a $\mathbb{k}$-linear map ("vector multiplication") satisfying total associativity

$$
\mu_{A}^{\left(n_{a}\right)}\left[\hat{\boldsymbol{a}}, \mu_{A}^{\left(n_{a}\right)}[\hat{\boldsymbol{b}}], \hat{\boldsymbol{c}}\right]=\text { invariant }
$$

where the second product $\mu_{A}^{\left(n_{a}\right)}$ can be on any place in brackets and $\hat{\boldsymbol{a}}, \hat{\boldsymbol{b}}, \hat{\boldsymbol{c}}$ are polyads;

4. The multiacton $\rho_{A}^{\left(r_{a}\right)}$ is compatible with vector and field operations $\left(\nu_{A}^{\left(m_{a}\right)}, \mu_{A}^{\left(n_{a}\right)} ; \nu_{k}^{\left(m_{k}\right)}, \mu_{k}^{\left(n_{k}\right)}\right)$.

Definition 3. We call the tuple $\left(m_{a}, n_{a} ; m_{k}, n_{k} ; r_{a}\right)$ an arity shape of the polyadic algebra $A$.

The compatibility of the multiaction $\rho_{A}^{\left(r_{a}\right)}$ ("linearity") consists of $[10,14]$ : 
1) Distributivity with respect to the $m_{a}$-ary vector addition $\nu_{A}^{\left(m_{a}\right)}(" \lambda(a+b)=\lambda a+\lambda b ")$

$$
\begin{aligned}
& \rho_{A}^{\left(r_{a}\right)}\left\{\lambda_{1}, \ldots \ldots, \lambda_{r_{a}} \mid \nu_{A}^{\left(m_{a}\right)}\left[a_{1}, \ldots, a_{m_{a}}\right]\right\} \\
& =\nu_{A}^{\left(m_{a}\right)}\left[\rho_{A}^{\left(r_{a}\right)}\left\{\lambda_{1}, \ldots \ldots, \lambda_{r_{a}} \mid a_{1}\right\}, \ldots, \rho_{A}^{\left(r_{a}\right)}\left\{\lambda_{1}, \ldots \ldots, \lambda_{r_{a}} \mid a_{m_{a}}\right\}\right] .
\end{aligned}
$$

2) Compatibility with $n_{a}$-ary "vector multiplication" $\mu_{A}^{\left(n_{a}\right)}("(\lambda a) \cdot(\mu b)=(\lambda \mu)(a \cdot b) ")$

$$
\begin{aligned}
& \mu_{A}^{\left(n_{a}\right)}\left[\rho_{A}^{\left(r_{a}\right)}\left\{\lambda_{1}, \ldots \ldots, \lambda_{r_{a}} \mid a_{1}\right\}, \ldots, \rho_{A}^{\left(r_{a}\right)}\left\{\lambda_{r_{a}\left(n_{a}-1\right)}, \ldots, \lambda_{r_{a} n_{a}} \mid a_{n_{a}}\right\}\right] \\
& =\rho_{A}^{\left(r_{a}\right)}\{\overbrace{\mu_{k}^{\left(n_{k}\right)}\left[\lambda_{1}, \ldots, \lambda_{m_{k}}\right], \ldots, \mu_{k}^{\left(n_{k}\right)}\left[\lambda_{m_{k}(\ell-1)}, \ldots \ldots, \lambda_{m_{k} \ell}\right]}^{\ell} \\
& \left.\lambda_{m_{k} \ell+1}, \ldots \ldots, \lambda_{r_{a} n_{a}} \mid \mu_{A}^{\left(n_{a}\right)}\left[a_{1}, \ldots, a_{n_{a}}\right]\right\} \\
& \ell\left(n_{k}-1\right)=r_{a}\left(n_{a}-1\right),
\end{aligned}
$$

where $\ell$ is an integer, and $\ell \leq r_{a} \leq \ell\left(n_{k}-1\right), 2 \leq n_{a} \leq n_{k}$.

3) Distributivity with respect to the $m_{k}$-ary field addition $\nu_{k}^{\left(m_{k}\right)}$ ("( $\left.\lambda+\mu\right) a=\lambda a+\mu a$ ")

$$
\begin{aligned}
& \rho^{\left(r_{a}\right)}\{\overbrace{\nu_{k}^{\left(m_{k}\right)}\left[\lambda_{1}, \ldots \ldots, \lambda_{m_{k}}\right], \ldots, \nu_{k}^{\left(m_{k}\right)}\left[\lambda_{m_{k}\left(\ell^{\prime}-1\right)}, \ldots \ldots, \lambda_{m_{k} \ell^{\prime}}\right]}^{\ell^{\prime}}, \lambda_{m_{k} \ell^{\prime}+1}, \ldots \ldots, \lambda_{r_{a} m_{a}} \mid a\} \\
& =\nu_{A}^{\left(m_{a}\right)}\left[\rho^{\left(r_{a}\right)}\left\{\lambda_{1}, \ldots \ldots, \lambda_{r_{a}} \mid a\right\}, \ldots, \rho^{\left(r_{a}\right)}\left\{\lambda_{r_{a}\left(m_{a}-1\right)}, \ldots \ldots, \lambda_{r_{a} m_{a}} \mid a\right\}\right], \\
& \ell^{\prime}\left(m_{k}-1\right)=r_{a}\left(m_{a}-1\right),
\end{aligned}
$$

where $\ell^{\prime}$ is an integer, and $\ell^{\prime} \leq r_{a} \leq \ell^{\prime}\left(m_{k}-1\right), 2 \leq m_{a} \leq m_{k}$.

4) Compatibility $n_{k}$-ary field multiplication $\mu_{k}^{\left(n_{k}\right)}(" \lambda(\mu a)=(\lambda \mu) a$ ")

$$
\begin{aligned}
& \rho_{A}^{\left(r_{a}\right)} \overbrace{\left\{\lambda_{1}, \ldots \ldots, \lambda_{r_{a}} \mid \ldots \rho_{A}^{\left(r_{a}\right)}\left\{\lambda_{r_{a}\left(n_{\rho}-1\right)}, \ldots \ldots, \lambda_{r_{a} n_{\rho}} \mid a\right\} \ldots\right\}}^{n_{\rho}} \\
& =\rho_{A}^{\left(r_{a}\right)}\{\overbrace{\mu_{k}^{\left(n_{k}\right)}\left[\lambda_{1}, \ldots \ldots, \lambda_{n_{k}}\right], \ldots, \mu_{k}^{\left(n_{k}\right)}\left[\lambda_{n_{k}\left(\ell^{\prime \prime}-1\right)}, \ldots \ldots, \lambda_{\left.n_{k} \ell^{\prime \prime}\right]}\right]}^{\ell^{\prime \prime}}, \lambda_{n_{k} \ell^{\prime \prime}+1}, \ldots, \lambda_{r_{a} n_{\rho}} \mid a\}
\end{aligned}
$$

where $\ell^{\prime \prime}$ is an integer, and $\ell^{\prime \prime} \leq r_{a} \leq \ell^{\prime \prime}\left(n_{k}-1\right), 2 \leq n_{\rho} \leq n_{k}$.

Remark 4. In the binary case, we have $m_{a}=n_{a}=m_{k}=n_{k}=n_{\rho}=2, r_{a}=\ell=\ell^{\prime}=\ell^{\prime \prime}=1$. The $n$-ary algebras $[11,12]$ have only one distinct arity $n_{a}=n$.

Definition 5. We call the triple $\left(\ell, \ell^{\prime}, \ell^{\prime \prime}\right)$ a $\ell$-arity shape of the polyadic algebra $A$.

Proposition 6. In the limiting $\ell$-arity shapes the arity shape of $A$ is determined by three integers $(m, n, r)$, such that:

1. For the maximal $\ell=\ell^{\prime}=\ell^{\prime \prime}=r_{a}$, the arity shape of the algebra and underlying field coincide

$$
\begin{aligned}
m_{a} & =m_{k}=m, \\
n_{a} & =n_{k}=n_{\rho}=n, \\
r_{a} & =r .
\end{aligned}
$$


2. For the minimal $\ell$-arities $\ell=\ell^{\prime}=\ell^{\prime \prime}=1$ it should be $r_{a} \mid\left(m_{k}-1\right)$ and $r_{a} \mid\left(n_{k}-1\right)$, and

$$
\begin{aligned}
m_{a} & =1+\frac{m-1}{r}, \\
n_{a} & =n_{\rho}=1+\frac{n-1}{r}, \\
m_{k} & =m, \\
n_{k} & =n, \\
r_{a} & =r .
\end{aligned}
$$

Proof. This follows directly from the compatibility conditions (14)-(18).

Proposition 7. If the multiaction $\rho_{A}^{\left(r_{a}\right)}$ is an ordinary action $K \times A \rightarrow A$, then all $\ell$-arities are minimal $\ell=\ell^{\prime}=\ell^{\prime \prime}=1$, and the arity shape of $A$ is determined by two integers $(m, n)$, such that the arities of the algebra and underlying field are equal, and the arity $n_{\rho}$ of the action semigroup $S_{\rho}$ is equal to the arity of multiplication in the underlying field

$$
\begin{aligned}
m_{a} & =m_{k}=m, \\
n_{a} & =n_{k}=n_{\rho}=n .
\end{aligned}
$$

As it was shown in [20], there exist zeroless and nonunital polyadic fields and rings. Therefore, the main difference with the binary algebras is the possible absence of a zero and/or unit in the polyadic field $\mathbb{k}^{\left(m_{k}, n_{k}\right)}$ and/or in the polyadic ring

$$
A_{\text {ring }}=A^{\left(m_{a}, n_{a}\right)}=\left\langle A \mid \nu_{A}^{\left(m_{a}\right)}, \mu_{A}^{\left(n_{a}\right)}\right\rangle,
$$

\begin{tabular}{|c|c|c|c|c|}
\hline${ }_{\mathbb{k}}\left(m_{k}, n_{k}\right) \quad A^{\left(m_{a}, n_{a}\right)}$ & $\begin{array}{l}z_{A} \\
e_{A}\end{array}$ & ${ }_{\text {no }}^{z} e_{A}$ & ${ }^{\text {no }} z_{A} A$ & $\begin{array}{ll}\text { no } & z_{A} \\
\text { no } & e_{A}\end{array}$ \\
\hline $\begin{array}{l}z_{k} \\
e_{k}\end{array}$ & $\begin{array}{l}\text { unital } A \\
\text { unital } \mathbb{k} \\
\end{array}$ & $\begin{array}{c}\text { nonunital } A \\
\text { unital } \mathbb{k}\end{array}$ & $\begin{array}{c}\text { unital zeroless } A \\
\text { unital } \mathbb{k}\end{array}$ & $\begin{array}{c}\text { nonunital zeroless } A \\
\text { unital } \mathbb{k}\end{array}$ \\
\hline $\begin{array}{c}z_{k} \\
\text { no } e_{k}\end{array}$ & $\begin{array}{c}\text { unital } A \\
\text { nonunital } \mathbb{k}\end{array}$ & $\begin{array}{c}\text { nonunital } A \\
\text { nonunital } \mathbb{k}\end{array}$ & $\begin{array}{c}\text { unital zeroless } A \\
\text { nonunital } \mathbb{k}\end{array}$ & $\begin{array}{c}\text { nonunital zeroless } A \\
\text { nonunital } \mathbb{k}\end{array}$ \\
\hline $\begin{array}{c}\text { no } z_{k} \\
e_{k}\end{array}$ & $\begin{array}{c}\text { unital } A \\
\text { unital zeroless } \mathbb{k}\end{array}$ & $\begin{array}{c}\text { nonunital } A \\
\text { unital zeroless } \mathbb{k}\end{array}$ & $\begin{array}{l}\text { unital zeroless } A \\
\text { unital zeroless } \mathbb{k}\end{array}$ & $\begin{array}{c}\text { nonunital zeroless } A \\
\text { unital zeroless } \mathbb{k}\end{array}$ \\
\hline $\begin{array}{ll}\text { no } & z_{k} \\
\text { no } & e_{k}\end{array}$ & $\begin{array}{c}\text { unital } A \\
\text { nonunital zeroless } \mathbb{k}\end{array}$ & $\begin{array}{c}\text { nonunital } A \\
\text { nonunital zeroless } \mathbb{k}\end{array}$ & $\begin{array}{c}\text { unital zeroless } A \\
\text { nonunital zeroless } \mathbb{k}\end{array}$ & $\begin{array}{c}\text { nonunital zeroless } A \\
\text { nonunital zeroless } \mathbb{k}\end{array}$ \\
\hline
\end{tabular}

and so the additional axioms are needed iff such elements exist. This was the reason we have started from Definition 2, where no existence of zeroes and units in $\mathbb{k}^{\left(m_{k}, n_{k}\right)}$ and $A_{\text {ring }}$ is implied.

If they exist, denote possible units and zeroes by $e_{k} \in \mathbb{k}^{\left(m_{k}, n_{k}\right)}, z_{k} \in \mathbb{k}^{\left(m_{k}, n_{k}\right)}$ and $e_{A} \in A^{\left(m_{a}, n_{a}\right)}, z_{A} \in$ $A^{\left(m_{a}, n_{a}\right)}$. In this way we have 4 choices for each $\mathbb{k}^{\left(m_{k}, n_{k}\right)}$ and $A^{\left(m_{a}, n_{a}\right)}$, and these 16 possible kinds of polyadic algebras are presented in TABLE 1 . The most exotic case is at the bottom right, where both $\mathbb{k}^{\left(m_{k}, n_{k}\right)}$ and $A^{\left(m_{a}, n_{a}\right)}$ are zeroless nonunital, which cannot exist in either binary algebras or $n$-ary algebras [11].

Table 1: Kinds of polyadic algebras depending on zeroes and units.

The standard case is that in the upper left corner, when both $\mathbb{k}^{\left(m_{k}, n_{k}\right)}$ and $A^{\left(m_{a}, n_{a}\right)}$ have a zero and unit. Example 8. Consider the ("kk-linear") associative polyadic algebra $A^{(3,3 ; 3,3 ; 2)}$ over the zeroless nonunital $(3,3)$ field $\mathbb{k}^{(3,3)}$ (from Example 1). The elements of $A$ are pairs $a=\left(\lambda, \lambda^{\prime}\right) \in \mathbb{k}^{(3,3)} \times \mathbb{k}^{(3,3)}$, and for them the ternary addition and ternary multiplication are defined by

$$
\begin{aligned}
& \mu_{A}^{(3)}\left[\left(\lambda_{1}, \lambda_{1}^{\prime}\right)\left(\lambda_{2}, \lambda_{2}^{\prime}\right)\left(\lambda_{3}, \lambda_{3}^{\prime}\right)\right]=\left(\lambda_{1} \lambda_{2}^{\prime} \lambda_{3}, \lambda_{1}^{\prime} \lambda_{2} \lambda_{3}^{\prime}\right) \\
& \nu_{A}^{(3)}\left[\left(\lambda_{1}, \lambda_{1}^{\prime}\right)\left(\lambda_{2}, \lambda_{2}^{\prime}\right)\left(\lambda_{3}, \lambda_{3}^{\prime}\right)\right]=\left(\lambda_{1}+\lambda_{2}+\lambda_{3}, \lambda_{1}^{\prime}+\lambda_{2}^{\prime}+\lambda_{3}^{\prime}\right), \quad \lambda_{i}, \lambda_{i}^{\prime} \in \mathbb{k}^{(3,3)}
\end{aligned}
$$

where operations on the r.h.s. are in $\mathbb{C}$. If we introduce an element $0 \notin \mathbb{k}^{(3,3)}$ with the property $0 \cdot \lambda=\lambda \cdot 0=0$, then (31)-(32) can be presented as the ordinary multiplication and addition of three anti-diagonal $2 \times 2$ formal matrices $\left(\begin{array}{cc}0 & \lambda \\ \lambda^{\prime} & 0\end{array}\right)$. There is no unit or zero in the ternary ring $\left\langle A \mid \nu_{A}^{(3)}, \mu_{A}^{(3)}\right\rangle$, but both $\left\langle A \mid \mu_{A}^{(3)}\right\rangle$ and $\left\langle A \mid \nu_{A}^{(3)}\right\rangle$ are ternary groups, because each $a=\left(i p / q, i p^{\prime} / q^{\prime}\right) \in A$ has the unique additive querelement $\tilde{a}=$ $\left(-i p / q,-i p^{\prime} / q^{\prime}\right)$ and the unique multiplicative querelement $\bar{a}=\left(-i q^{\prime} / p^{\prime},-i q / p\right)$. The 2-place action ("2-scalar product") is defined by $\rho^{(2)}\left(\lambda_{1}, \lambda_{2} \mid\left(\lambda, \lambda^{\prime}\right)\right)=\left(\lambda_{1} \lambda_{2} \lambda, \lambda_{1} \lambda_{2} \lambda^{\prime}\right)$. The arity shape (see Definition 5$)$ of this zeroless nonunital polyadic algebra $A^{(3,3 ; 3,3 ; 2)}$ is $(2,2,2)$, and the compatibilities (13)-(19) hold. 


\section{Polyadic analog of the functions on group}

In the search for a polyadic version of the algebra of $\mathbb{k}$-valued functions (which is isomorphic and dual to the corresponding group algebra) we can not only have more complicated arity shapes than in the binary case, but also the exotic possibility that the arities of the field and group are different as can be possible for multiplace functions.

Let us consider a $n_{g}$-ary group $G=G^{\left(n_{g}\right)}=\left\langle G \mid \mu_{g}^{\left(n_{g}\right)}\right\rangle$, which does not necessarily contain the identity $e_{g}$, and where each element is querable (see (4)). Now we introduce the set $A_{f}$ of multiplace ( $s$-place) functions $f_{i}\left(g_{1}, \ldots, g_{s}\right)$ (of finite support) which take value in the polyadic field $\mathbb{k}^{\left(m_{k}, n_{k}\right)}$ such that $f_{i}: G^{s} \rightarrow K$. To endow $A_{f}$ with the structure of a polyadic associative algebra (10), we should consistently define the $m_{k}$-ary addition $\nu_{f}^{\left(m_{k}\right)}: A_{f}^{\left(m_{k}\right)} \rightarrow A_{f}, n_{k}$-ary multiplication ("convolution") $\mu_{f}^{\left(n_{k}\right)}: A_{f}^{\left(n_{k}\right)} \rightarrow A_{f}$ and the multiaction $\rho_{f}^{\left(r_{f}\right)}: K^{r_{f}} \times A_{f} \rightarrow A_{f}$ ("scalar multiplication"). Thus we write for the algebra of $\mathbb{k}$-valued functions

$$
F_{\mathrm{k}}(G)=\left\langle A_{f} \mid \nu_{f}^{\left(m_{k}\right)}, \mu_{f}^{\left(n_{k}\right)} ; \nu_{k}^{\left(m_{k}\right)}, \mu_{k}^{\left(n_{k}\right)} ; \rho_{f}^{\left(r_{f}\right)}\right\rangle .
$$

The simplest operation here is the addition of the $\mathbb{k}$-valued functions which, obviously, coincides with the field addition $\nu_{f}^{\left(m_{k}\right)}=\nu_{k}^{\left(m_{k}\right)}$.

Construction 9. Because all arguments of the multiacton $\rho_{f}^{\left(r_{f}\right)}$ are in the field, the only possibility for the r.h.s. is its multiplication (similar to the regular representation)

$$
\rho_{f}^{\left(r_{f}\right)}\left(\lambda_{1}, \ldots, \lambda_{r_{f}} \mid f\right)=\mu_{k}^{\left(n_{k}\right)}\left[\lambda_{1}, \ldots, \lambda_{r_{f}}, f\right], \quad \lambda_{i} \in K, \quad f \in A_{f},
$$

and in addition we have the arity shape relation

$$
n_{k}=r_{f}+1,
$$

which is satisfied "automatically" in the binary case.

The polyadic analog of $\mathbb{k}$-valued function convolution (" $\left(f_{1} * f_{2}\right)(g)=\Sigma_{h_{1} h_{2}=g} f_{1}\left(h_{1}\right) f_{2}\left(h_{2}\right)$ "), which is denoted by $\mu_{f}^{\left(n_{k}\right)}$ here, while the sum in the field is $\nu_{k}^{\ell_{\nu}\left(m_{k}-1\right)+1}$, where $\ell_{\nu}$ is the "number of additions", can be constructed according to the arity rules from $[10,14]$.

Definition 10. The polyadic convolution of $s$-place $\mathbb{k}$-valued functions is defined as the admissible polyadic sum of $\ell_{\nu}\left(m_{k}-1\right)+1$ products

$$
\begin{aligned}
& \mu_{f}^{\left(n_{k}\right)}\left[f_{1}\left(g_{1}, \ldots, g_{s}\right), \ldots, f_{n_{k}}\left(g_{1}, \ldots, g_{s}\right)\right]= \\
& \left(\nu_{k}^{\left(m_{k}\right)}\right)^{\circ \ell_{\nu}} \quad\left[\mu_{k}^{\left(n_{k}\right)}\left[f_{1}\left(h_{1}, \ldots, h_{s}\right), \ldots, f_{n_{k}}\left(h_{s\left(n_{k}-1\right)}, \ldots, h_{s n_{k}}\right)\right]\right],
\end{aligned}
$$

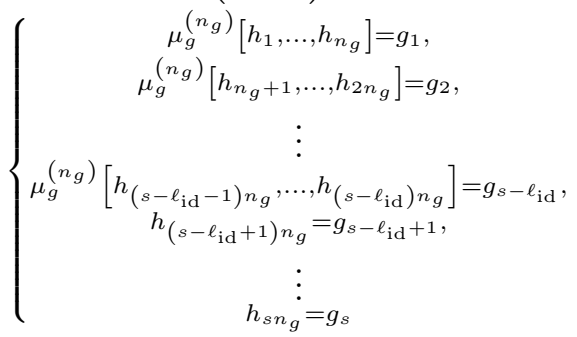

where $\ell_{\text {id }}$ is the number of intact elements in the determining equations ( $h_{1} h_{2}=g$ ") under the field sum $\nu_{k}$. The arity shape is determined by

$$
s n_{k}=\left(s-\ell_{\mathrm{id}}\right) n_{g}+\ell_{\mathrm{id}},
$$

which gives the connection between the field and the group arities.

Example 11. If $n_{g}=3, n_{k}=2, m_{k}=3, s=2, \ell_{\mathrm{id}}=1$, then we obtain the arity changing polyadic convolution

$$
\begin{aligned}
& \mu_{f}^{(2)}\left[f_{1}\left(g_{1}, g_{2}\right), f_{2}\left(g_{1}, g_{2}\right)\right]= \\
& \left(\nu_{k}^{(3)}\right)^{\circ \ell_{\nu}}\left[\mu_{k}^{(2)}\left[f_{1}\left(h_{1}, h_{2}\right), f_{2}\left(h_{3}, h_{4}\right)\right]\right], \\
& \left\{\begin{array}{c}
\mu_{g}^{(3)}\left[h_{1}, h_{2}, h_{3}\right]=g_{1}, \\
\mu_{g}^{(4)}=g_{2}
\end{array}\right.
\end{aligned}
$$


where the $\ell_{\nu}$ ternary additions are taken on the support. Now the multiaction (34) is one-place

$$
\rho_{f}^{(1)}(\lambda \mid f)=\mu_{k}^{(2)}[\lambda, f], \quad \lambda \in K, \quad f \in A_{f},
$$

as it follows from (35).

Remark 12. The general polyadic convolution (36) is inspired by the main heteromorphism equation (5.14) and the arity changing formula (5.15) of [14]. The graphical dependence of the field arity $n_{k}$ on the number of places $s$ is similar to that on Figure 1, and the "quantization" rules (following from the solutions of (37) in integers) are in TABLE 1 there.

Proposition 13. The multiplication (36) is associative.

Proof. This follows from the associativity quiver technique of [14] applied to the polyadic convolution.

Corollary 14. The $\mathbb{k}$-valued multiplace functions $\left\{f_{i}\right\}$ form a polyadic associative algebra $F_{\mathrm{k}}(G)$.

\section{"Diagrammatic" description}

Here we formulate the polyadic algebra axioms in the more customary "diagrammatic" form using the polyadic tensor products and mappings between them (denoted by bold corresponding letters). Informally, the $\mathbb{k}$-linearity is already "automatically encoded" by the polyadic tensor algebra over $\mathbb{k}$, and therefore the axioms already contain the algebra multiplication (but not the scalar multiplication).

Let us denote the $\mathbb{k}$-linear algebra multiplication map by $\boldsymbol{\mu}^{(n)}\left(\mu^{(n)} \equiv \mu_{A}^{\left(n_{a}\right)}\right.$ from (10)) defined as

$$
\boldsymbol{\mu}^{(n)} \circ\left(a_{1} \otimes \ldots \otimes a_{n}\right)=\mu^{(n)}\left[a_{1}, \ldots, a_{n}\right], \quad a_{1}, \ldots, a_{n} \in A .
$$

Definition 15 (Algebra associativity axiom). A polyadic (associative $n$-ary) algebra (or $\mathbb{k}$-algebra) is a vector space $A_{\text {vect }}$ over the polyadic field $\mathbb{k}(11)$ with the $\mathbb{k}$-linear algebra multiplication map

$$
A^{(n)}=\left\langle A_{\text {vect }} \mid \boldsymbol{\mu}^{(n)}\right\rangle, \quad \boldsymbol{\mu}^{(n)}: A^{\otimes n} \rightarrow A,
$$

which is totally associative

$$
\begin{aligned}
\boldsymbol{\mu}^{(n)} \circ\left(\mathrm{id}_{A}^{\otimes(n-1-i)} \otimes \boldsymbol{\mu}^{(n)} \otimes \mathrm{id}_{A}^{\otimes i}\right) & =\boldsymbol{\mu}^{(n)} \circ\left(\mathrm{id}_{A}^{\otimes(n-1-j)} \otimes \boldsymbol{\mu}^{(n)} \otimes \mathrm{id}_{A}^{\otimes j}\right), \\
\forall i, j & =0, \ldots n-1, i \neq j, \quad \operatorname{id}_{A}: A \rightarrow A,
\end{aligned}
$$

such that the diagram

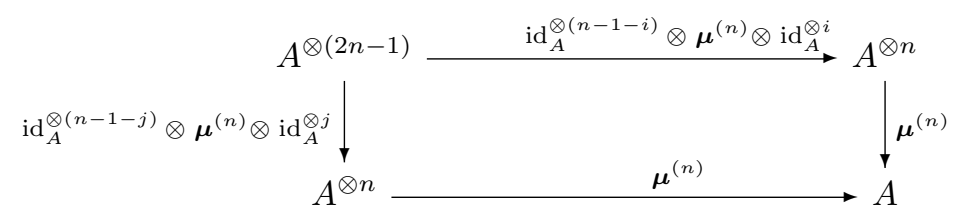

commutes.

Definition 16. A polyadic algebra $A^{(n)}$ is called totally commutative, if

$$
\boldsymbol{\mu}^{(n)}=\boldsymbol{\mu}^{(n)} \circ \boldsymbol{\tau}_{n},
$$

where $\boldsymbol{\tau}_{n} \in S_{n}$, and $S_{n}$ is the symmetry permutation group on $n$ elements.

Remark 17. Initially, there are no other axioms in the definition of a polyadic algebra, because polyadic fields and vector spaces do not necessarily contain zeroes and units (see TABLE 1).

A special kind of polyadic algebra can appear, when the multiplication is "iterated" from lower arity ones, which is one of 3 kinds of arity changing for polyadic systems [14].

Definition 18. A polyadic multiplication is called derived, if the map $\boldsymbol{\mu}_{d e r}^{(n)}$ is $\ell_{\mu}$-iterated from the maps $\boldsymbol{\mu}_{0}^{\left(n_{0}\right)}$ of lower arity $n_{0}<n$

$$
\boldsymbol{\mu}_{d e r}^{(n)}=\overbrace{\boldsymbol{\mu}_{0}^{\left(n_{0}\right)} \circ\left(\boldsymbol{\mu}_{0}^{\left(n_{0}\right)} \circ \ldots\left(\boldsymbol{\mu}_{0}^{\left(n_{0}\right)} \otimes \mathrm{id}^{\otimes\left(n_{0}-1\right)}\right) \otimes \ldots \otimes \mathrm{id}^{\otimes\left(n_{0}-1\right)}\right)}^{\ell_{\mu}},
$$

where

$$
n=\ell_{\mu}\left(n_{0}-1\right)+1, \quad \ell_{\mu} \geq 2,
$$

and $\ell_{\mu}$ is the "number of iterations". 
Example 19. In the ternary case $n=3$ and $n_{0}=2$, we have $\boldsymbol{\mu}_{d e r}^{(3)}=\boldsymbol{\mu}_{0}^{(2)} \circ\left(\boldsymbol{\mu}_{0}^{(2)} \otimes\right.$ id $)$, which in the "elementwise" description is $\left[a_{1}, a_{2}, a_{3}\right]_{d e r}=a_{1} \cdot\left(a_{2} \cdot a_{3}\right)$, where $\mu_{d e r}^{(3)}=[,,]_{d e r}$ and $\mu_{0}^{(2)}=(\cdot)$.

Introduce a $\mathbb{k}$-linear multiaction map $\boldsymbol{\rho}^{(r)}$ corresponding to the multiaction $\rho^{(r)} \equiv \rho_{A}^{\left(r_{a}\right)}$ (7) (by analogy with (40)) as

$$
\boldsymbol{\rho}^{(r)} \circ\left(\lambda_{1} \otimes \ldots \otimes \lambda_{r} \otimes a\right)=\rho^{(r)}\left(\lambda_{1}, \ldots, \lambda_{r} \mid a\right), \quad \lambda_{1}, \ldots, \lambda_{r} \in K, a \in A .
$$

Let $\mathbb{k}$ and $A^{(n)}$ both be unital, then we can construct a $\mathbb{k}$-linear polyadic unit map $\boldsymbol{\eta}$ by "polyadizing" " $\mu \circ(\eta \otimes \mathrm{id})=\mathrm{id}$ " and the scalar product " $\lambda a=\rho(\lambda \mid a)=\eta(\lambda) a$ " with " $\eta\left(e_{k}\right)=e_{a}$ ", using the normalization (8), and taking into account the standard identification $\mathbb{k}^{\otimes r} \otimes A \cong A[23]$.

Definition 20 (Algebra unit axiom). The unital polyadic algebra $A^{(n)}$ (41) contains in addition a $\mathbb{k}$-linear polyadic (right) unit map $\boldsymbol{\eta}^{(r, n)}: K^{\otimes r} \rightarrow A^{\otimes(n-1)}$ satisfying

$$
\boldsymbol{\mu}^{(n)} \circ\left(\boldsymbol{\eta}^{(r, n)} \otimes \operatorname{id}_{A}\right)=\boldsymbol{\rho}^{(r)},
$$

such that the diagram

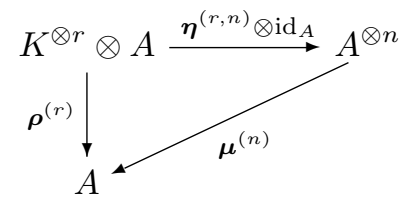

commutes.

The normalization of the multiaction (8) gives the corresponding normalization of the map $\boldsymbol{\eta}^{(r, n)}$ (instead of " $\eta\left(e_{k}\right)=e_{a}$ ")

$$
\boldsymbol{\eta}^{(r, n)} \circ(\overbrace{e_{k} \otimes \ldots \otimes e_{k}}^{r})=\overbrace{e_{a} \otimes \ldots \otimes e_{a}}^{n-1}
$$

Assertion 21. In the "elementwise" description the polyadic unit $\eta^{(r, n)}$ of $A^{(n)}$ is a $(n-1)$-valued function of $r$ arguments.

Proposition 22. The polyadic unit map $\boldsymbol{\eta}^{(r, n)}$ is (polyadically) multiplicative in the following sense

$$
\overbrace{\boldsymbol{\mu}^{(n)} \circ \ldots \circ \boldsymbol{\mu}^{(n)}}^{r} \circ(\overbrace{\boldsymbol{\eta}^{(r, n)} \otimes \ldots \otimes \boldsymbol{\eta}^{(r, n)}}^{r(n-1)+1})=\boldsymbol{\eta}^{(r, n)} \circ\left(\mu_{k}^{\left(n_{k}\right)}\right)^{\circ \ell} \text {. }
$$

Proof. This follows from the compatibility of the multiaction with the "vector multiplication" (14) and the relation between corresponding arities (15), such that the number of arguments ("scalars" $\lambda_{i}$ ) in r.h.s. becomes $\ell\left(n_{k}-1\right)+1=r(n-1)+1$, where $\ell$ is an integer.

Introduce a "derived" version of the polyadic unit by analogy with the neutral sequence (3).

Definition 23. The $\mathbb{k}$-linear derived polyadic unit (neutral unit sequence) of $n$-ary algebra $A^{(n)}$ is the set $\hat{\boldsymbol{\eta}}^{(r)}=\left\{\boldsymbol{\eta}_{i}^{(r)}\right\}$ of $n-1$ maps $\boldsymbol{\eta}_{i}^{(r)}: K^{\otimes r} \rightarrow A, i=1, \ldots, n-1$, satisfying

$$
\boldsymbol{\mu}^{(n)} \circ\left(\boldsymbol{\eta}_{1}^{(r)} \otimes \ldots \otimes \boldsymbol{\eta}_{n-1}^{(r)} \otimes \operatorname{id}_{A}\right)=\boldsymbol{\rho}^{(r)}
$$

where $\operatorname{id}_{A}$ can be on any place. If $\boldsymbol{\eta}_{1}^{(r)}=\ldots=\boldsymbol{\eta}_{n-1}^{(r)}=\boldsymbol{\eta}_{0}^{(r)}$, we call $\boldsymbol{\eta}_{0}^{(r)}$ the strong derived polyadic unit. Formally (comparing (48) and (52)), we can write

$$
\boldsymbol{\eta}_{\text {der }}^{(r, n)}=\boldsymbol{\eta}_{1}^{(r)} \otimes \ldots \otimes \boldsymbol{\eta}_{n-1}^{(r)} .
$$

The normalization of the maps $\boldsymbol{\eta}_{i}^{(r)}$ is given by

$$
\boldsymbol{\eta}_{i}^{(r)} \circ(\overbrace{e_{k} \otimes \ldots \otimes e_{k}}^{r})=e_{a}, \quad i=1, \ldots, n-1, \quad e_{a} \in A, \quad e_{k} \in K,
$$

and in the "elementwise" description $\eta_{i}^{(r)}$ is a function of $r$ arguments, satisfying

$$
\eta_{i}^{(r)}\left(\lambda_{1}, \ldots, \lambda_{r}\right)=\rho^{(r)}\left\{\lambda_{1}, \ldots, \lambda_{r} \mid e_{a}\right\}, \quad \lambda_{i} \in K,
$$

where $\rho^{(r)}$ is the multiaction $(7)$. 
Definition 24. A polyadic associative algebra $A_{d e r}^{(n)}=\left\langle A_{\text {vect }} \mid \boldsymbol{\mu}_{d e r}^{(n)}, \boldsymbol{\eta}_{d e r}^{(r, n)}\right\rangle$ is called derived from $A_{0}^{\left(n_{0}\right)}=$ $\left\langle A_{\text {vect }} \mid \boldsymbol{\mu}_{0}^{\left(n_{0}\right)}, \boldsymbol{\eta}_{0}^{\left(r, n_{0}\right)}\right\rangle$, if (45) holds and further

$$
\boldsymbol{\eta}_{d e r}^{(r, n)}=\overbrace{\boldsymbol{\eta}_{0}^{\left(r, n_{0}\right)} \otimes \ldots \otimes \boldsymbol{\eta}_{0}^{\left(r, n_{0}\right)}}^{\ell_{\mu}}
$$

is true, where $\boldsymbol{\eta}_{0}^{\left(r, n_{0}\right)}=\overbrace{\boldsymbol{\eta}_{0}^{(r)} \otimes \ldots \otimes \boldsymbol{\eta}_{0}^{(r)}}^{n_{0}-1}$ (formally, because id $\mathrm{id}_{A}$ in (52) can be on any place).

The particular case $n=3$ and $r=1$ was considered in [24,25] (with examples).

Invertibility in a polyadic algebra is not connected with the unit or zero (as in $n$-ary groups [17]), but is determined by the querelement (4). Introduce the corresponding mappings for the subsets of the additively querable elements $A_{\text {quer }}^{(\text {add })} \subseteq A$ and the multiplicatively querable elements $A_{\text {quer }}^{(\text {mult })} \subseteq A$.

Definition 25. In the polyadic algebra $A^{(m, n)}$, the additive quermap $\boldsymbol{q}_{a d d}: A_{q u e r}^{(a d d)} \rightarrow A_{q u e r}^{(a d d)}$ is defined by

$$
\boldsymbol{\nu}^{(m)} \circ\left(\mathrm{id}_{A}^{\otimes(m-1)} \otimes \boldsymbol{q}_{a d d}\right) \circ \boldsymbol{D}_{a}^{(m)}=\mathrm{id}_{A},
$$

and the multiplicative quermap $\boldsymbol{q}_{\text {mult }}: A_{\text {quer }}^{(\text {mult })} \rightarrow A_{\text {quer }}^{(\text {mult })}$ is defined by

$$
\boldsymbol{\mu}^{(n)} \circ\left(\operatorname{id}_{A}^{\otimes(n-1)} \otimes \boldsymbol{q}_{m u l t}\right) \circ \boldsymbol{D}_{a}^{(n)}=\mathrm{id}_{A},
$$

where $\boldsymbol{D}_{a}^{(n)}: A \rightarrow A^{\otimes n}$ is the diagonal map given by $a \rightarrow \overbrace{a \otimes \ldots \otimes a}^{n}$, while $\boldsymbol{q}_{a d d}$ and $\boldsymbol{q}_{\text {mult }}$ can be on any place. They send an element to the additive querelement $a \stackrel{\boldsymbol{q}_{\text {add }}}{\mapsto} \tilde{a}, a \in A_{\text {quer }}^{(\text {add }} \subseteq A$ and multiplicative querelement $a \stackrel{\boldsymbol{q}_{\text {mult }}}{\mapsto} \bar{a}, a \in A_{\text {quer }}^{(\text {mult })} \subseteq A$ (see (4)), such that the diagrams
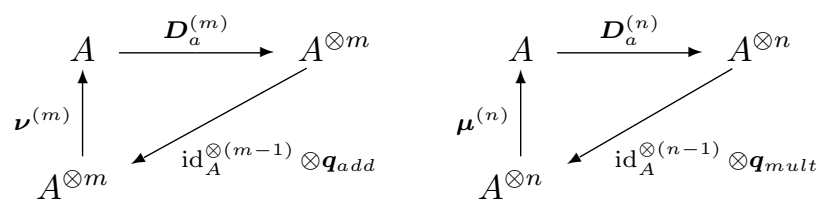

commute.

Example 26. For the polyadic algebra $A^{(3,3 ; 3,3 ; 2)}$ from Example 8 all elements are additively and multiplicatively querable, and so the sets of querable elements coincide $A_{q u e r}^{(\text {add })}=A_{q u e r}^{(\text {mult })}=A$. The additive quermap $\boldsymbol{q}_{a d d}$ and multiplicative quermap $\boldsymbol{q}_{\text {mult }}$ act as follows (the operations are in $\mathbb{C}$ )

$$
\begin{aligned}
& \left(i \frac{p}{q}, i \frac{p^{\prime}}{q^{\prime}}\right) \stackrel{\boldsymbol{q}_{a d d}}{\longmapsto}\left(-i \frac{p}{q},-i \frac{p^{\prime}}{q^{\prime}}\right), \\
& \left(i \frac{p}{q}, i \frac{p^{\prime}}{q^{\prime}}\right) \stackrel{\boldsymbol{q}_{\text {mult }}}{\mapsto}\left(-i \frac{q^{\prime}}{p^{\prime}},-i \frac{q}{p}\right), \quad i^{2}=-1, \quad p, q \in \mathbb{Z}^{\text {odd }} .
\end{aligned}
$$

Example 27. The polyadic field $\mathbb{k}^{\left(m_{k}, n_{k}\right)}$ is a polyadic algebra over itself. We identify $A=K, \mu_{A}^{(n)}=\mu_{k}^{\left(n_{k}\right)}$, and the multiplication is defined by the multiaction as

$$
\boldsymbol{\mu}^{(n)} \circ\left(\lambda_{1} \otimes \ldots \otimes \lambda_{r} \otimes \lambda\right)=\rho^{(r)}\left(\lambda_{1}, \ldots, \lambda_{r} \mid \lambda\right) .
$$

Therefore, we have the additional arity conditions

$$
n=r+1=n_{k}
$$

which are trivially satisfied in the binary case. Now the polyadic unit map $\boldsymbol{\eta}^{(r, n)}(50)$ is the identity in each tensor component.

\section{Medial map and polyadic permutations}

Recall that the binary medial map for the tensor product of algebras (as vector spaces)

$$
\tau_{\text {medial }}:\left(A_{1} \otimes A_{2}\right) \otimes\left(A_{1} \otimes A_{2}\right) \rightarrow\left(A_{1} \otimes A_{1}\right) \otimes\left(A_{2} \otimes A_{2}\right)
$$


is defined by (evaluation)

$$
\left(a_{1}^{(1)} \otimes a_{1}^{(2)}\right) \otimes\left(a_{2}^{(1)} \otimes a_{2}^{(2)}\right) \stackrel{\tau_{\text {medial }}}{\longmapsto}\left(a_{1}^{(1)} \otimes a_{2}^{(1)}\right) \otimes\left(a_{1}^{(2)} \otimes a_{2}^{(2)}\right) .
$$

It is obvious that

$$
\tau_{\text {medial }}=\mathrm{id}_{A} \otimes \tau_{o p} \otimes \mathrm{id}_{A},
$$

where $\tau_{o p}: A_{1} \otimes A_{2} \rightarrow A_{1} \otimes A_{2}$ is the permutation of 2 elements (twist/flip) of the tensor product, such that $a^{(1)} \otimes a^{(2)} \stackrel{\tau_{o p}}{\mapsto} a^{(2)} \otimes a^{(1)}, a^{(1)} \in A_{1}, a^{(2)} \in A_{2}, \tau_{o p} \in S_{2}$. This may be presented (65) in the matrix form

$$
\bigotimes(\boldsymbol{a})_{2 \times 2} \stackrel{\tau_{\text {medial }}}{\mapsto} \bigotimes\left(\boldsymbol{a}^{T}\right)_{2 \times 2}, \quad \bigotimes(\boldsymbol{a})_{2 \times 2}=\bigotimes\left(\begin{array}{ll}
a_{1}^{(1)} & a_{1}^{(2)} \\
a_{2}^{(1)} & a_{2}^{(2)}
\end{array}\right)
$$

where $T$ is the ordinary matrix transposition.

Let us apply (64) to arbitrary tensor products. By analogy, if we have a tensor product of $m n$ elements (of any nature) grouped by $n$ elements (e.g. $m$ elements from $n$ different vector spaces), as in (65), (67), we can write the tensor product in the $(m \times n)$-matrix form (cf. (3.18)-(3.19) in [14])

$$
\bigotimes(\boldsymbol{a})_{m \times n}=\bigotimes\left(\begin{array}{cccc}
a_{1}^{(1)} & a_{1}^{(2)} & \ldots & a_{1}^{(n)} \\
a_{2}^{(1)} & a_{2}^{(2)} & \ldots & a_{2}^{(n)} \\
\vdots & \vdots & \vdots & \vdots \\
a_{m}^{(1)} & a_{m}^{(2)} & \ldots & a_{m}^{(n)}
\end{array}\right)
$$

Definition 28. The polyadic medial map $\boldsymbol{\tau}_{\text {medial }}^{(n, m)}:\left(A^{\otimes n}\right)^{\otimes m} \rightarrow\left(A^{\otimes m}\right)^{\otimes n}$ is defined as the transposition of the tensor product matrix (68) by the evaluation (cf. the binary case (65))

$$
\bigotimes(\boldsymbol{a})_{m \times n} \stackrel{\tau_{\text {medial }}^{(n, m)}}{\substack{\text { eqial } \\ \text { (a) }}} \bigotimes\left(\boldsymbol{a}^{T}\right)_{n \times m}
$$

We can extend the mediality concept $[26,27]$ to polyadic algebras using the medial map. If we have an algebra with $n$-ary multiplication (40), then the mediality relation follows from (68) with $m=n$ and contains $(n+1)$ multiplications acting on $n^{2}$ elements.

Definition 29. A $\mathbb{k}$-linear polyadic algebra $A^{(n)}(41)$ is called medial, if its $n$-ary multiplication map satisfies the relation

$$
\boldsymbol{\mu}^{(n)} \circ\left(\left(\boldsymbol{\mu}^{(n)}\right)^{\otimes n}\right)=\boldsymbol{\mu}^{(n)} \circ\left(\left(\boldsymbol{\mu}^{(n)}\right)^{\otimes n}\right) \circ \boldsymbol{\tau}_{\text {medial }}^{(n, n)},
$$

where $\boldsymbol{\tau}_{\text {medial }}^{(n, n)}$ is given by (69), or in the manifest elementwise form (evaluation)

$$
\begin{aligned}
& \mu^{(n)}\left[\mu^{(n)}\left[a_{1}^{(1)}, a_{1}^{(2)}, \ldots, a_{1}^{(n)}\right], \mu^{(n)}\left[a_{2}^{(1)}, a_{2}^{(2)}, \ldots, a_{2}^{(n)}\right], \ldots, \mu^{(n)}\left[a_{n}^{(1)}, a_{n}^{(2)}, \ldots, a_{n}^{(n)}\right]\right] \\
& =\mu^{(n)}\left[\mu^{(n)}\left[a_{1}^{(1)}, a_{2}^{(1)}, \ldots, a_{n}^{(1)}\right], \mu^{(n)}\left[a_{1}^{(2)}, a_{2}^{(2)}, \ldots, a_{n}^{(2)}\right], \ldots, \mu^{(n)}\left[a_{1}^{(n)}, a_{2}^{(n)}, \ldots, a_{n}^{(n)}\right]\right] .
\end{aligned}
$$

Let us "polyadize" the binary twist map $\tau_{o p}$ from (66), which can be suitable for operations with polyadic tensor products. Informally, we can interpret (66), as "omitting the fixed points" of the binary medial map $\boldsymbol{\tau}_{\text {medial }}$, and denote this procedure by " $\tau_{o p}=\tau_{\text {medial }} \backslash \mathrm{id}$ ".

Definition 30. A (medially allowed) $\ell_{\tau}$-place polyadic twist map $\boldsymbol{\tau}_{o p}^{\left(\ell_{\tau}\right)}$ is defined by

$$
\text { " } \tau_{o p}^{\left(\ell_{\tau}\right)}=\tau_{\text {medial }}^{(n, m)} \backslash \mathrm{id} ",
$$

where $\ell_{\tau}=m n-k_{\text {fixed }}$, and $k_{\text {fixed }}$ is the number of fixed points of the medial map $\tau_{\text {medial }}^{(n, m)}$.

Assertion 31. If $m \neq n$, then $\ell_{\tau}=m n-2$. If $m=n$, then the polyadic twist map $\boldsymbol{\tau}_{o p}^{\left(\ell_{\tau}\right)}$ is the reflection

$$
\tau_{o p}^{\left(\ell_{\tau}\right)} \circ \tau_{o p}^{\left(\ell_{\tau}\right)}=\mathrm{id}_{A}
$$

and $\ell_{\tau}=n(n-1)$ 
Table 2: Number of places $\ell_{\tau}$ in the polyadic twist map $\tau_{o p}^{\left(\ell_{\tau}\right)}$.

\begin{tabular}{||c||c|c|c|c|c|c||}
\hline \hline$m$ & 2 & 3 & 4 & 5 & 6 & 7 \\
\hline \hline 2 & $\mathbf{2}$ & $\mathbf{4}$ & $\mathbf{6}$ & $\mathbf{8}$ & $\mathbf{1 0}$ & $\mathbf{1 2}$ \\
\hline 3 & $\mathbf{4}$ & $\mathbf{6}$ & $\mathbf{1 0}$ & $\mathbf{1 3}$ & $\mathbf{1 6}$ & $\mathbf{1 9}$ \\
\hline 4 & $\mathbf{6}$ & $\mathbf{1 0}$ & $\mathbf{1 2}$ & $\mathbf{1 8}$ & $\mathbf{2 2}$ & $\mathbf{2 6}$ \\
\hline 5 & $\mathbf{8}$ & $\mathbf{1 3}$ & $\mathbf{1 8}$ & $\mathbf{2 0}$ & $\mathbf{2 8}$ & $\mathbf{3 3}$ \\
\hline 6 & $\mathbf{1 0}$ & $\mathbf{1 6}$ & $\mathbf{2 2}$ & $\mathbf{2 8}$ & $\mathbf{3 0}$ & $\mathbf{4 0}$ \\
\hline 7 & $\mathbf{1 2}$ & $\mathbf{1 9}$ & $\mathbf{2 6}$ & $\mathbf{3 3}$ & $\mathbf{4 0}$ & $\mathbf{4 2}$ \\
\hline \hline
\end{tabular}

Proof. This follows from the matrix form (68) and (69).

Therefore the number of places $\ell_{\tau}$ is "quantized" and for lowest $m, n$ is presented in TABLE 2.

This generalizes the binary twist in a more unique way, which gives polyadic commutativity.

Remark 32. The polyadic twist map $\boldsymbol{\tau}_{o p}^{\left(\ell_{\tau}\right)}$ is one element of the symmetry permutation group $S_{\ell_{\tau}}$ which is fixed by the medial map $\boldsymbol{\tau}_{\text {medial }}^{(n, m)}$ and the special condition (72), and it therefore respects polyadic tensor product operations.

Example 33. In the matrix representation we have

$$
\begin{aligned}
& \left.\tau_{o p}^{(4)}\right|_{n=3, m=2}=\left(\begin{array}{cccc}
0 & 0 & 1 & 0 \\
1 & 0 & 0 & 0 \\
0 & 0 & 0 & 1 \\
0 & 1 & 0 & 0
\end{array}\right),\left.\tau_{o p}^{(6)}\right|_{n=3, m=3}=\left(\begin{array}{cccccc}
0 & 0 & 1 & 0 & 0 & 0 \\
0 & 0 & 0 & 0 & 1 & 0 \\
1 & 0 & 0 & 0 & 0 & 0 \\
0 & 0 & 0 & 0 & 0 & 1 \\
0 & 1 & 0 & 0 & 0 & 0 \\
0 & 0 & 0 & 1 & 0 & 0
\end{array}\right), \\
& \left.\tau_{o p}^{(6)}\right|_{n=4, m=2}=\left(\begin{array}{cccccc}
0 & 0 & 0 & 1 & 0 & 0 \\
1 & 0 & 0 & 0 & 0 & 0 \\
0 & 0 & 0 & 0 & 1 & 0 \\
0 & 1 & 0 & 0 & 0 & 0 \\
0 & 0 & 0 & 0 & 0 & 1 \\
0 & 0 & 1 & 0 & 0 & 0
\end{array}\right) .
\end{aligned}
$$

The introduction of the polyadic twist gives us the possibility to generalize (in a way consistent with the medial map) the notion of the opposite algebra.

Definition 34. For a polyadic algebra $A^{(n)}=\left\langle A \mid \boldsymbol{\mu}^{(n)}\right\rangle$, an opposite polyadic algebra

$$
A_{o p}^{(n)}=\left\langle A \mid \boldsymbol{\mu}^{(n)} \circ \boldsymbol{\tau}_{o p}^{(n)}\right\rangle
$$

exists if the number of places for the polyadic twist map (which coincides in (76) with the arity of algebra multiplication $\ell_{\tau}=n$ ) is allowed (see TABLE 2).

Definition 35. A polyadic algebra $A^{(n)}$ is called medially commutative, if

$$
\boldsymbol{\mu}_{o p}^{(n)} \equiv \boldsymbol{\mu}^{(n)} \circ \boldsymbol{\tau}_{o p}^{(n)}=\boldsymbol{\mu}^{(n)},
$$

where $\boldsymbol{\tau}_{o p}^{(n)}$ is the medially allowed polyadic twist map.

\section{Tensor product of polyadic algebras}

Let us consider a polyadic tensor product $\bigotimes_{i=1}^{n} A_{i}^{(n)}$ of $n$ polyadic associative $n$-ary algebras $A_{i}^{(n)}=$ $\left\langle A_{i} \mid \boldsymbol{\mu}_{i}^{(n)}\right\rangle, i=1, \ldots, n$, such that (see (40))

$$
\boldsymbol{\mu}_{i}^{(n)} \circ\left(a_{1}^{(i)} \otimes \ldots \otimes a_{n}^{(i)}\right)=\mu_{A_{i}}^{(n)}\left[a_{1}^{(i)}, \ldots, a_{n}^{(i)}\right], \quad a_{1}^{(i)}, \ldots, a_{n}^{(i)} \in A_{i}, \quad \mu_{A_{i}}^{(n)}: A_{i}^{(n)} \rightarrow A_{i} .
$$

To endow $\bigotimes_{i=1}^{n} A_{i}^{(n)}$ with the structure of an algebra, we will use the medial map $\tau_{\text {medial }}^{(n, m)}(69)$. 
Proposition 36. The tensor product of $n$ associative $n$-ary algebras $A_{i}^{(n)}$ has the structure of the polyadic algebra $A_{\otimes}^{(n)}=\left\langle\bigotimes_{i=1}^{n} A_{i}^{(n)} \mid \boldsymbol{\mu}_{\otimes}\right\rangle$, which is associative (cf. (42))

$$
\begin{aligned}
& \boldsymbol{\mu}_{\otimes} \circ\left(\mathrm{id}_{A_{\otimes}}^{\otimes(n-1-i)} \otimes \boldsymbol{\mu}_{\otimes} \otimes \mathrm{id}_{A_{\otimes}}^{\otimes i}\right)=\boldsymbol{\mu}_{\otimes} \circ\left(\mathrm{id}_{A_{\otimes}}^{\otimes(n-1-j)} \otimes \boldsymbol{\mu}_{\otimes} \otimes \mathrm{id}_{A_{\otimes}}^{\otimes j}\right), \\
& \forall i, j=0, \ldots n-1, i \neq j, \quad \operatorname{id}_{A_{\otimes}}: A_{1}^{\otimes n} \otimes \ldots \otimes A_{n}^{\otimes n} \rightarrow A,
\end{aligned}
$$

if

$$
\boldsymbol{\mu}_{\otimes}=\left(\boldsymbol{\mu}_{1}^{(n)} \otimes \ldots \otimes \boldsymbol{\mu}_{n}^{(n)}\right) \circ \boldsymbol{\tau}_{\text {medial }}^{(n, n)} .
$$

Proof. We act by the multiplication map $\boldsymbol{\mu}_{\otimes}$ on the element's tensor product matrix (68) and obtain

$$
\begin{aligned}
& \boldsymbol{\mu}_{\otimes} \circ\left(\left(a_{1}^{(1)} \otimes a_{1}^{(2)} \otimes \ldots \otimes a_{1}^{(n)}\right) \otimes \ldots \otimes\left(a_{n}^{(1)} \otimes a_{n}^{(2)} \otimes \ldots \otimes a_{n}^{(n)}\right)\right) \\
& =\left(\boldsymbol{\mu}_{1}^{(n)} \otimes \ldots \otimes \boldsymbol{\mu}_{n}^{(n)}\right) \circ \boldsymbol{\tau}_{\text {medial }}^{(n, n)} \circ\left(\left(a_{1}^{(1)} \otimes a_{1}^{(2)} \otimes \ldots \otimes a_{1}^{(n)}\right) \otimes \ldots \otimes\left(a_{n}^{(1)} \otimes a_{n}^{(2)} \otimes \ldots \otimes a_{n}^{(n)}\right)\right) \\
& =\left(\boldsymbol{\mu}_{1}^{(n)} \otimes \ldots \otimes \boldsymbol{\mu}_{n}^{(n)}\right) \circ\left(\left(a_{1}^{(1)} \otimes a_{2}^{(1)} \otimes \ldots \otimes a_{n}^{(1)}\right) \otimes \ldots \otimes\left(a_{1}^{(n)} \otimes a_{2}^{(n)} \otimes \ldots \otimes a_{n}^{(n)}\right)\right) \\
& =\mu_{1}^{(n)}\left[a_{1}^{(1)}, a_{2}^{(1)}, \ldots, a_{n}^{(1)}\right] \otimes \ldots \otimes \mu_{n}^{(n)}\left[a_{1}^{(n)}, a_{2}^{(n)}, \ldots, a_{n}^{(n)}\right]
\end{aligned}
$$

which proves that $\boldsymbol{\mu}_{\otimes}$ is indeed a polyadic algebra multiplication. To prove the associativity (79) we repeat the same derivation (81) twice and show that the result is independent of $i, j$.

If all $A_{i}^{(n)}$ have their polyadic unit map $\boldsymbol{\eta}_{i}^{(r, n)}$ defined by (48) and acting as (50), then we have

Proposition 37. The polyadic unit map of $A_{\otimes}^{(n)}$ is $\boldsymbol{\eta}_{\otimes}: K^{\otimes n r} \rightarrow A_{1}^{\otimes(n-1)} \otimes \ldots \otimes A_{n}^{\otimes(n-1)}$ acting as

$$
\boldsymbol{\eta}_{\otimes} \circ(\overbrace{e_{k} \otimes \ldots \otimes e_{k}}^{n r})=(\overbrace{e_{a_{1}} \otimes \ldots \otimes e_{a_{1}}}^{n-1}) \otimes \ldots \otimes(\overbrace{e_{a_{n}} \otimes \ldots \otimes e_{a_{n}}}^{n-1}) .
$$

Assertion 38. The polyadic unit of $A_{\otimes}^{(n)}$ is a $\left(n^{2}-n\right)$-valued function of nr arguments.

Note that concepts of tensor product and derived polyadic algebras are different.

\section{Heteromorphisms of polyadic associative algebras}

The standard homomorphism between binary associative algebras is defined as a linear map $\varphi$ which "commutes" with the algebra multiplications (" $\varphi \circ \boldsymbol{\mu}_{1}=\boldsymbol{\mu}_{2} \circ(\varphi \otimes \varphi)$ "). In the polyadic case there exists the possibility to change arity of the algebras, and for that, one needs to use the heteromorphisms (or multiplace maps) introduced in [14]. Let us consider two polyadic $\mathbb{k}$-algebras $A_{1}^{\left(n_{1}\right)}=\left\langle A_{1} \mid \boldsymbol{\mu}_{1}^{\left(n_{1}\right)}\right\rangle$ and $A_{2}^{\left(n_{2}\right)}=\left\langle A_{2} \mid \boldsymbol{\mu}_{2}^{\left(n_{2}\right)}\right\rangle$ (over the same polyadic field $\mathbb{k}$ ).

Definition 39. A heteromorphism between two polyadic $\mathbb{k}$-algebras $A_{1}^{\left(n_{1}\right)}$ and $A_{2}^{\left(n_{2}\right)}$ (of different arities $n_{1}$ and $\left.n_{2}\right)$ is a $s$-place $\mathbb{k}$-linear map $\boldsymbol{\Phi}_{s}^{\left(n_{1}, n_{2}\right)}: A_{1}^{\otimes s} \rightarrow A_{2}$, such that

$$
\boldsymbol{\Phi}_{s}^{\left(n_{1}, n_{2}\right)} \circ(\overbrace{\boldsymbol{\mu}_{1}^{\left(n_{1}\right)} \otimes \ldots \otimes \boldsymbol{\mu}_{1}^{\left(n_{1}\right)}}^{s-\ell_{\text {id }}} \otimes \overbrace{\mathrm{id}_{A_{1}} \otimes \ldots \otimes \operatorname{id}_{A_{1}}}^{\ell_{\text {id }}})=\boldsymbol{\mu}_{2}^{\left(n_{2}\right)} \circ(\overbrace{\Phi_{s}^{\left(n_{1}, n_{2}\right)} \otimes \ldots \otimes \boldsymbol{\Phi}_{s}^{\left(n_{1}, n_{2}\right)}}^{n_{2}}),
$$

and the diagram

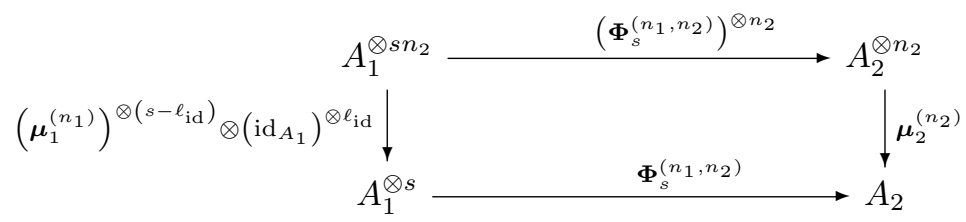

commutes. The arities satisfy

$$
s n_{2}=n_{1}\left(s-\ell_{\mathrm{id}}\right)+\ell_{\mathrm{id}},
$$

where $0 \leq \ell_{\text {id }} \leq s-1$ is an integer (the number of "intact elements" of $A_{1}$ ), and therefore $2 \leq n_{2} \leq n_{1}$. 
Assertion 40. If $\ell_{\mathrm{id}}=0$ (there are no "intact elements"), then the (s-place) heteromorphism does not change the arity of the polyadic algebra.

Definition 41. A homomorphism between two polyadic $\mathbb{k}$-algebras $A_{1}^{(n)}$ and $A_{2}^{(n)}$ (of the same arity or equiary) is a 1-place $\mathbb{k}$-linear map $\boldsymbol{\Phi}^{(n)}=\boldsymbol{\Phi}_{s=1}^{(n, n)}: A_{1} \rightarrow A_{2}$, such that

$$
\boldsymbol{\Phi}^{(n)} \circ \boldsymbol{\mu}_{1}^{(n)}=\boldsymbol{\mu}_{2}^{(n)} \circ(\overbrace{\Phi^{(n)} \otimes \ldots \otimes \Phi^{(n)}}^{n}),
$$

and the diagram

commutes.

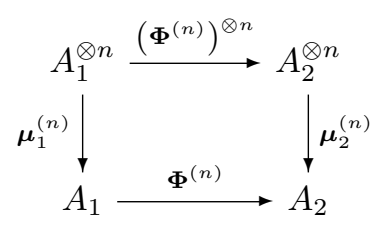

The above definitions do not include the behavior of the polyadic unit under heteromorphism, because a polyadic associative algebra need not contain a unit. However, if both units exist, this will lead to strong arity restrictions.

Proposition 42. If in $\mathbb{k}$-algebras $A_{1}^{\left(n_{1}\right)}$ and $A_{2}^{\left(n_{2}\right)}$ (of arities $n_{1}$ and $n_{2}$ ) there exist both polyadic units (48) $\boldsymbol{\eta}_{1}^{\left(r, n_{1}\right)}: K^{\otimes r} \rightarrow A_{1}^{\otimes\left(n_{1}-1\right)}$ and $\boldsymbol{\eta}_{2}^{\left(r, n_{2}\right)}: K^{\otimes r} \rightarrow A_{2}^{\otimes\left(n_{2}-1\right)}$, then

1. The heteromorphism (83) connects them as

$$
\overbrace{\boldsymbol{\eta}_{2}^{\left(r, n_{2}\right)} \otimes \ldots \otimes \boldsymbol{\eta}_{2}^{\left(r, n_{2}\right)}}^{s}=(\overbrace{\boldsymbol{\Phi}_{s}^{\left(n_{1}, n_{2}\right)} \otimes \ldots \otimes \boldsymbol{\Phi}_{s}^{\left(n_{1}, n_{2}\right)}}^{n_{1}-1}) \circ(\overbrace{\boldsymbol{\eta}_{1}^{\left(r, n_{1}\right)} \otimes \ldots \otimes \boldsymbol{\eta}_{1}^{\left(r, n_{1}\right)}}^{s}),
$$

and the diagram

commutes.

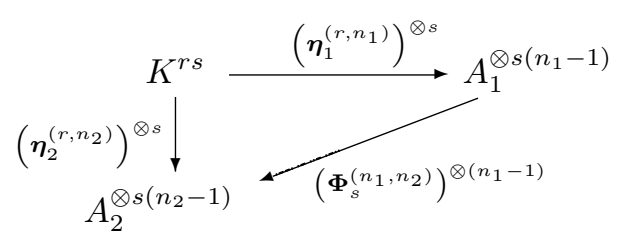

2. The number of "intact elements" is fixed by its maximum value

$$
\ell_{\mathrm{id}}=s-1,
$$

such that in the l.h.s. of (83) there is only one multiplication $\boldsymbol{\mu}_{1}^{\left(n_{1}\right)}$.

3. The number of places $s$ in the heteromorphism $\boldsymbol{\Phi}_{s}^{\left(n_{1}, n_{2}\right)}$ is fixed by the arities of the polyadic algebras

$$
s\left(n_{2}-1\right)=n_{1}-1 \text {. }
$$

Proof. Using (85) we obtain $s\left(n_{2}-1\right)=\left(s-\ell_{\text {id }}\right)\left(n_{1}-1\right)$, then the $\left(n_{1}-1\right)$ power of the heteromorphism $\mathbf{\Phi}_{s}^{\left(n_{1}, n_{2}\right)}$ maps $A_{1}^{\otimes s\left(n_{1}-1\right)} \rightarrow A_{2}^{\otimes\left(n_{1}-1\right)}$, and we have $s-\ell_{\mathrm{id}}=1$, which, together with (85), gives (90), and (91).

\section{Structure constants}

Let $A^{(n)}$ be a finite-dimensional polyadic algebra (10) having the basis $e_{i} \in A, i=1, \ldots, N$, where $N$ is its dimension as a polyadic vector space

$$
A_{v e c t}=\left\langle A, K \mid \nu^{(m)} ; \nu_{k}^{\left(m_{k}\right)}, \mu_{k}^{\left(n_{k}\right)} ; \rho^{(r)}\right\rangle
$$

where we denote $\nu^{(m)}=\nu_{A}^{\left(m_{a}\right)}$ (see (9) and (11), here $N=d_{v}$ ). In the binary case " $a=\sum_{i} \lambda^{(i)} e_{i}$ ", any element $a \in A$ is determined by the number $N_{\lambda}$ of scalars $\lambda \in K$, which coincides with the algebra dimension $N_{\lambda}=N$, because $r=1$. In the polyadic case, it can be that $r>1$, and moreover with $m \geq 2$ the admissible number of "words" (in the expansion of $a$ by $e_{i}$ ) is "quantized", such that $1, m, 2 m-1,3 m-2, \ldots \ell_{N}(m-1)+1$, where $\ell_{N} \in \mathbb{N}_{0}$ is the "number of additions". So we have 
Definition 43. In $N$-dimensional $n$-ary algebra $A^{(n)}$ (with $m$-ary addition and $r$-place "scalar" multiplication) the expansion of any element $a \in A$ by the basis $\left\{e_{i} \mid i=1, \ldots, N\right\}$ is

$$
a=\left(\nu^{(m)}\right)^{\circ \ell_{N}}\left[\rho^{(r)}\left\{\lambda_{1}^{(1)}, \ldots, \lambda_{r}^{(1)} \mid e_{1}\right\}, \ldots, \rho^{(r)}\left\{\lambda_{1}^{(N)}, \ldots, \lambda_{r}^{(N)} \mid e_{N}\right\}\right],
$$

and is determined by $N_{\lambda} \in \mathbb{N}$ "scalars", where

$$
\begin{aligned}
N_{\lambda} & =r N, \\
N & =\ell_{N}(m-1)+1, \quad \ell_{N} \in \mathbb{N}_{0}, \quad N \in \mathbb{N}, \quad m \geq 2 .
\end{aligned}
$$

In the binary case $m=2$, the dimension $N$ of an algebra is not restricted and is a natural number, because, $N=\ell_{N}+1$.

Assertion 44. The dimension of n-ary algebra $A^{(n)}$ having $m$-ary addition is not arbitrary, but "quantized" and can only have the following values for $m \geq 3$

$$
\begin{array}{ll}
m=3, & N=1,3,5, \ldots, 2 \ell_{N}+1, \\
m=4, & N=1,4,7, \ldots, 3 \ell_{N}+1, \\
m=5, & N=1,5,9, \ldots, 4 \ell_{N}+1,
\end{array}
$$

Proof. It follows from (95) and demanding that the "number of additions" $\ell_{N}$ is natural or zero.

In a similar way, by considering a product of the basis elements, which can also be expanded in the basis " $e_{i} e_{j}=\sum_{k} \chi_{(i, j)}^{(k)} e_{k}$ ", we can define a polyadic analog of the structure constants $\chi_{(i, j)}^{(k)} \in K$.

Definition 45. The polyadic structure constants $\chi_{r,\left(i_{1}, \ldots i_{n}\right)}^{(j)} \in K, i_{1}, \ldots i_{n}, j=1, \ldots, n$ of the $N$-dimensional $n$-ary algebra $A^{(n)}$ (with $m$-ary addition $\nu^{(m)}$ and $r$-place multiaction $\rho^{(r)}$ ) are defined by the expansion of the $n$-ary product of the basis elements $\left\{e_{i} \mid i=1, \ldots, N\right\}$ as

$$
\begin{aligned}
& \mu^{(n)}\left[e_{i_{1}}, \ldots, e_{i_{n}}\right] \\
& =\left(\nu^{(m)}\right)^{\circ \ell_{N}}\left[\rho^{(r)}\left\{\chi_{1,\left(i_{1}, \ldots i_{n}\right)}^{(1)}, \ldots, \chi_{r,\left(i_{1}, \ldots i_{n}\right)}^{(1)} \mid e_{1}\right\}, \ldots, \rho^{(r)}\left\{\chi_{1,\left(i_{1}, \ldots i_{n}\right)}^{(N)}, \ldots, \chi_{r,\left(i_{1}, \ldots i_{n}\right)}^{(N)} \mid e_{N}\right\}\right],
\end{aligned}
$$

where

$$
\begin{aligned}
N_{\chi} & =r N^{n+1}, \quad N, N_{\chi} \in \mathbb{N} \\
N & =\ell_{N}(m-1)+1, \quad \ell_{N} \in \mathbb{N}_{0}, \quad m \geq 2 .
\end{aligned}
$$

As in the binary case, we have

Corollary 46. The algebra multiplication $\mu^{(n)}$ of $A^{(n)}$ is fully determined by the $r N^{n+1}$ polyadic structure constants $\chi_{r,\left(i_{1}, \ldots i_{n}\right)}^{(j)} \in K$.

Contrary to the binary case $m=2$, when $N_{\chi}$ can be any natural number, we now have

Assertion 47. The number of the polyadic structure constants $N_{\chi}$ of the finite-dimensional n-ary algebra $A^{(n)}$ with m-ary addition and r-place multiaction is not arbitrary, but "quantized" according to

$$
N_{\chi}=r\left(\ell_{N}(m-1)+1\right)^{n+1}, \quad r \in \mathbb{N}, \quad \ell_{N} \in \mathbb{N}_{0}, \quad m, n \geq 2 .
$$

Proof. This follows from (100) and "quantization" of the algebra dimension N, see Assertion 44.

\section{POLYADIC COALGEBRAS}

\section{Motivation}

The standard motivation for introducing the comultiplication is from representation theory $[28,29]$. The first examples come from so-called addition formulas for special functions ("anciently" started from $\sin / \cos$ ), which actually arise from representations of groups [5,30].

In brief (and informally), let $\boldsymbol{\pi}$ be a finite-dimensional representation of a group $G$ in a vector space $V$ over a field $k$, such that

$$
\boldsymbol{\pi}(g h)=\boldsymbol{\pi}(g) \boldsymbol{\pi}(h), \quad \boldsymbol{\pi}: G \rightarrow \text { End } V, \quad g, h \in G .
$$


In some basis of $V$ the matrix elements $\pi_{i j}(g)$ satisfy $\pi_{i j}(g h)=\sum_{k} \pi_{i k}(g) \pi_{k j}(h)$ (from (103)) and span a finite dimensional vector space $C_{\pi}$ of functions with a basis $e_{\pi_{m}}$ as $f_{\pi}=\sum_{m} \alpha_{m} e_{\pi_{m}}, f_{\pi} \in C_{\pi}$. Now (103) gives $f_{\pi}(g h)=\sum_{m, n} \beta_{m n} e_{\pi_{m}}(g) e_{\pi_{n}}(h), f_{\pi} \in C_{\pi}$. If we omit the evaluation, it can be written in the vector space $C_{\pi}$ using an additional linear map $\Delta_{\pi}: C_{\pi} \rightarrow C_{\pi} \otimes C_{\pi}$, in the following way

$$
\Delta_{\pi}\left(f_{\pi}\right)=\sum_{m, n} \beta_{m n} e_{\pi_{m}} \otimes e_{\pi_{n}} \in C_{\pi} \otimes C_{\pi}
$$

Thus, to any finite-dimensional representation $\pi$ one can define the map $\Delta_{\pi}$ of vector spaces $C_{\pi}$ to functions on a group, called a comultiplication.

It is important that all the above operations are binary, and the defining formula for comultiplication (104) is fully determined by the definition of a representation (103).

The polyadic analog of a representation was introduced and studied in [14]. In the case of multiplace representations, arities of the initial group and its representation can be different. Indeed, let $G^{(n)}=\left\langle G \mid \mu_{G}^{(n)}\right\rangle$, $\mu_{G}^{(n)}: G^{\times n} \rightarrow G$, be a $n$-ary group and $G_{\text {End } V}^{\left(n^{\prime}\right)}=\left\langle\{\right.$ End $\left.V\} \mid \mu_{E}^{\left(n^{\prime}\right)}\right\rangle, \mu_{E}^{\left(n^{\prime}\right)}:(\text { End } V)^{\times n^{\prime}} \rightarrow$ End $V$, is a $n^{\prime}$-ary group of endomorphisms of a polyadic vector space $V(9)$. In [14] $G_{\text {End } V}^{\left(n^{\prime}\right)}$ was considered as a derived one, while here we do not restrict it in this way.

Definition 48. A polyadic (multiplace) representation of $G^{(n)}$ in $V$ is a $s$-place mapping

$$
\Pi_{s}^{\left(n, n^{\prime}\right)}: G^{\times s} \rightarrow \text { End } V
$$

satisfying the associativity preserving heteromorphism equation [14]

$$
\begin{aligned}
& \boldsymbol{\Pi}_{s}^{\left(n, n^{\prime}\right)}(\overbrace{\mu_{G}^{(n)}\left[g_{1}, \ldots g_{n}\right], \ldots, \mu_{G}^{(n)}\left[g_{n\left(s-\ell_{\mathrm{id}}^{\prime}-1\right)}, \ldots, g_{n\left(s-\ell_{\mathrm{id}}^{\prime}\right)}\right]}^{s-\ell_{\mathrm{id}}^{\prime}}, \ldots, \overbrace{g_{n\left(s-\ell_{\mathrm{id}}^{\prime}\right)+1}, \ldots, g_{n\left(s-\ell_{\mathrm{id}}^{\prime}\right)+\ell_{\mathrm{id}}^{\prime}}}^{\ell_{\mathrm{id}}^{\prime}}) \\
& =\mu_{E}^{\left(n^{\prime}\right)}\left[\boldsymbol{\Pi}_{s}^{\left(n, n^{\prime}\right)}\left(g_{1}, \ldots g_{s}\right), \ldots, \boldsymbol{\Pi}_{s}^{\left(n, n^{\prime}\right)}\left(g_{s\left(n^{\prime}-1\right)}, \ldots g_{s n^{\prime}}\right)\right]
\end{aligned}
$$

such that the diagram

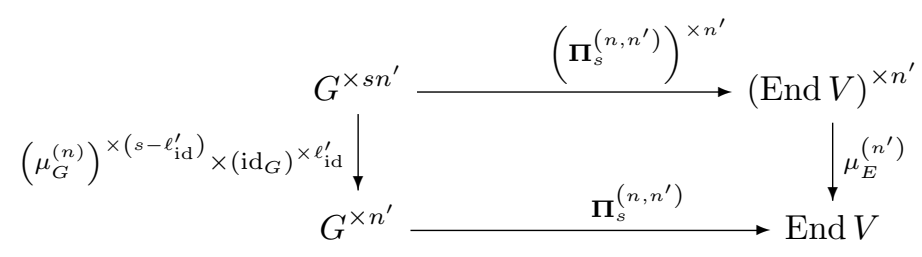

commutes, and the arity changing formula

$$
s n^{\prime}=n\left(s-\ell_{\mathrm{id}}^{\prime}\right)+\ell_{\mathrm{id}}^{\prime}
$$

where $\ell_{\mathrm{id}}^{\prime}$ is the number of "intact elements" in l.h.s. of (106), $0 \leq \ell_{\mathrm{id}}^{\prime} \leq s-1,2 \leq n^{\prime} \leq n$.

Remark 49. Particular examples of 2-place representations of ternary groups $(s=2$, which change arity from $n=3$ to $\left.n^{\prime}=2\right)$, together with their matrix representations, were presented in [14,31].

\section{Polyadic comultiplication}

Our motivations say that in constructing a polyadic analog of the comultiplication, one should not only "reverse arrows", but also pay thorough attention to arities.

Assertion 50. The arity of polyadic comultiplication coincides with the arity of the representation and can differ from the arity of the polyadic algebra.

Proof. It follows from (103), (104) and (105).

Let us consider a polyadic vector space over the polyadic field $\mathbb{k}^{\left(m_{k}, n_{k}\right)}$ as (see (11))

$$
C_{v e c t}=\left\langle C, K \mid \nu_{C}^{\left(m_{c}\right)} ; \nu_{k}^{\left(m_{k}\right)}, \mu_{k}^{\left(n_{k}\right)} ; \rho_{C}^{\left(r_{c}\right)}\right\rangle
$$

where $\nu_{C}^{\left(m_{c}\right)}: C^{\times m_{c}} \rightarrow C$ is $m_{c^{-}}$ary addition and $\rho_{C}^{\left(r_{c}\right)}: K^{\times r_{c}} \times C \rightarrow C$ is $r_{c}$-place action (see (7)). 
Definition 51. A polyadic ( $n^{\prime}$-ary) comultiplication is a $\mathbb{k}$-linear map $\Delta^{\left(n^{\prime}\right)}: C \rightarrow C^{\otimes n^{\prime}}$.

Definition 52. A polyadic (coassociative) coalgebra (or $\mathbb{k}$-coalgebra) is the polyadic vector space $C_{v e c t}$ equipped with the polyadic comultiplication

$$
C=C^{\left(n^{\prime}\right)}=\left\langle C_{v e c t} \mid \boldsymbol{\Delta}^{\left(n^{\prime}\right)}\right\rangle
$$

which is (totally) coassociative

$$
\begin{aligned}
\left(\operatorname{id}_{C}^{\otimes\left(n^{\prime}-1-i\right)} \otimes \boldsymbol{\Delta}^{\left(n^{\prime}\right)} \otimes \mathrm{id}_{C}^{\otimes i}\right) \circ \boldsymbol{\Delta}^{\left(n^{\prime}\right)} & =\left(\operatorname{id}_{C}^{\otimes(n-1-j)} \otimes \boldsymbol{\Delta}^{\left(n^{\prime}\right)} \otimes \mathrm{id}_{C}^{\otimes j}\right) \circ \boldsymbol{\Delta}^{\left(n^{\prime}\right)}, \\
\forall i, j & =0, \ldots n-1, i \neq j, \quad \operatorname{id}_{C}: C \rightarrow C,
\end{aligned}
$$

and such that the diagram

$$
\left.\operatorname{id}_{C}^{\otimes\left(n^{\prime}-1-j\right)} \otimes \Delta^{\left(n^{\prime}\right)} \otimes \operatorname{id}_{C}^{\otimes j}\right|_{C} ^{\otimes\left(2 n^{\prime}-1\right) \longleftarrow{i^{\prime}}_{C}^{\otimes\left(n^{\prime}-1-i\right)} \otimes \Delta^{\left(n^{\prime}\right)} \otimes \operatorname{id}_{C}^{\otimes i}} C^{\otimes n^{\prime}} \uparrow_{\Delta^{\left(n^{\prime}\right)}}
$$

commutes (cf.(43)).

Definition 53. A polyadic coalgebra $C^{\left(n^{\prime}\right)}$ is called totally co-commutative, if

$$
\boldsymbol{\Delta}^{\left(n^{\prime}\right)}=\tau_{n^{\prime}} \circ \boldsymbol{\Delta}^{\left(n^{\prime}\right)},
$$

where $\boldsymbol{\tau}_{n^{\prime}} \in S_{n^{\prime}}$, and $S_{n^{\prime}}$ is the permutation symmetry group on $n^{\prime}$ elements.

Definition 54. A polyadic coalgebra $C^{\left(n^{\prime}\right)}$ is called medially co-commutative, if

$$
\boldsymbol{\Delta}_{c o p}^{\left(n^{\prime}\right)} \equiv \boldsymbol{\tau}_{o p}^{\left(n^{\prime}\right)} \circ \boldsymbol{\Delta}^{\left(n^{\prime}\right)}=\boldsymbol{\Delta}^{\left(n^{\prime}\right)}
$$

where $\boldsymbol{\tau}_{o p}^{\left(n^{\prime}\right)}$ is the medially allowed polyadic twist map (72).

There are no other axioms in the definition of a polyadic coalgebra, following the same reasoning as for a polyadic algebra: the possible absence of zeroes and units (see TABLE 1). Obviously, in a polyadic coalgebra $C^{\left(n^{\prime}\right)}$, there is no "unit element", because there is no multiplication, and a polyadic analog of counit can be only defined, when the underlying field $\mathbb{k}^{\left(m_{k}, n_{k}\right)}$ is unital (which is not always the case [20]).

By analogy with (6), introduce the $\ell^{\prime}$-coiterated $n^{\prime}$-ary comultiplication by

$$
\left(\boldsymbol{\Delta}^{\left(n^{\prime}\right)}\right)^{\circ \ell^{\prime}}=\overbrace{\left(\operatorname{id}_{C}^{\otimes\left(n^{\prime}-1\right)} \otimes \ldots\left(\operatorname{id}_{C}^{\otimes\left(n^{\prime}-1\right)} \boldsymbol{\Delta}^{\left(n^{\prime}\right)}\right) \ldots \circ \Delta^{\left(n^{\prime}\right)}\right) \circ \boldsymbol{\Delta}^{\left(n^{\prime}\right)}}^{\ell^{\prime}}, \quad \ell^{\prime} \in \mathbb{N} .
$$

Therefore, the admissible length of any co-word is fixed ("quantized") as $\ell^{\prime}\left(n^{\prime}-1\right)+1$, but not arbitrary, as in the binary case.

Let us introduce a co-analog of the derived $n$-ary multiplication (45) by

Definition 55. A polyadic comultiplication $\Delta_{d e r}^{\left(n^{\prime}\right)}$ is called derived, if it is $\ell_{d^{-}}$-coiterated from the comultiplication $\boldsymbol{\Delta}_{0}^{\left(n_{0}^{\prime}\right)}$ of lower arity $n_{0}^{\prime}<n^{\prime}$

$$
\boldsymbol{\Delta}_{d e r}^{\left(n^{\prime}\right)}=\overbrace{\left(\operatorname{id}_{C}^{\otimes\left(n_{0}^{\prime}-1\right)} \otimes \ldots\left(\operatorname{id}_{C}^{\otimes\left(n_{0}^{\prime}-1\right)} \Delta_{0}^{\left(n_{0}^{\prime}\right)}\right) \ldots \circ \Delta_{0}^{\left(n_{0}^{\prime}\right)}\right) \circ \boldsymbol{\Delta}_{0}^{\left(n_{0}^{\prime}\right)}}^{\ell_{d}}
$$

or

$$
\boldsymbol{\Delta}_{d e r}^{\left(n^{\prime}\right)}=\left(\boldsymbol{\Delta}_{0}^{\left(n_{0}^{\prime}\right)}\right)^{\circ \ell^{\prime}}
$$

where

$$
n^{\prime}=\ell_{d}\left(n_{0}^{\prime}-1\right)+1
$$

and $\ell_{d} \geq 2$ is the "number of coiterations". 
The standard coiterations of $\boldsymbol{\Delta}$ are binary and restricted by $n_{0}^{\prime}=2$ ( [1]).

Example 56. The matrix coalgebra generated by the basis $e_{i j}, i, j=1, \ldots, N$ of $\operatorname{Mat}_{N}(\mathbb{C})$ with the binary coproduct $\Delta_{0}^{(2)}\left(e_{i j}\right)=\sum_{k} e_{i k} \otimes e_{k j}$ (see, e.g., [2]) can be extended to the derived ternary coalgebra by $\Delta_{d e r}^{(3)}\left(e_{i j}\right)=\sum_{k, l} e_{i k} \otimes e_{k l} \otimes e_{l j}$, such that (116) becomes $\boldsymbol{\Delta}_{\text {der }}^{(3)}=\left(\operatorname{id}_{C} \otimes \boldsymbol{\Delta}_{0}^{(2)}\right) \boldsymbol{\Delta}_{0}^{(2)}=\left(\boldsymbol{\Delta}_{0}^{(2)} \otimes \mathrm{id}_{C}\right) \boldsymbol{\Delta}_{0}^{(2)}$.

Example 57. Let us consider the ternary coalgebra $\left\langle C \mid \boldsymbol{\Delta}^{(3)}\right\rangle$ generated by two elements $\{a, b\} \in C$ with the von Neumann regular looking comultiplication

$$
\Delta^{(3)}(a)=a \otimes b \otimes a, \quad \Delta^{(3)}(b)=b \otimes a \otimes b .
$$

It is easy to check that $\boldsymbol{\Delta}^{(3)}$ is coassociative and nonderived.

Definition 58. A polyadic coalgebra $C^{\left(n^{\prime}\right)}(110)$ is called co-medial, if its $n^{\prime}$-ary multiplication map satisfies the relation

$$
\left(\left(\boldsymbol{\Delta}^{\left(n^{\prime}\right)}\right)^{\otimes n^{\prime}}\right) \circ \boldsymbol{\Delta}^{\left(n^{\prime}\right)}=\boldsymbol{\tau}_{\text {medial }}^{\left(n^{\prime}, n^{\prime}\right)} \circ\left(\left(\boldsymbol{\Delta}^{\left(n^{\prime}\right)}\right)^{\otimes n^{\prime}}\right) \circ \boldsymbol{\Delta}^{\left(n^{\prime}\right)},
$$

where $\boldsymbol{\tau}_{\text {medial }}^{\left(n^{\prime}, n^{\prime}\right)}$ is the polyadic medial map given by (68)-(69).

Introduce a $\mathbb{k}$-linear $r^{\prime}$-place action map $\overline{\boldsymbol{\rho}}^{\left(r^{\prime}\right)}: K^{\otimes r^{\prime}} \otimes C \rightarrow C$ corresponding to $\rho_{C}^{\left(r_{c}\right)}$ by (see (47))

$$
\overline{\boldsymbol{\rho}}^{\left(r^{\prime}\right)} \circ\left(\lambda_{1} \otimes \ldots \otimes \lambda_{r^{\prime}} \otimes c\right)=\rho_{C}^{\left(r^{\prime}\right)}\left(\lambda_{1}, \ldots, \lambda_{r^{\prime}} \mid c\right), \quad \lambda_{1}, \ldots, \lambda_{r^{\prime}} \in K, c \in C .
$$

Let $\mathbb{k}^{\left(m_{k}, n_{k}\right)}$ be unital with unit $e_{k}$.

Definition 59. A $\mathbb{k}$-linear $r^{\prime}$-place coaction map $\boldsymbol{\sigma}^{\left(r^{\prime}\right)}: C \rightarrow K^{\otimes r^{\prime}} \otimes C$ is defined by

$$
c \mapsto \overbrace{e_{k} \otimes \ldots \otimes e_{k}}^{r^{\prime}} \otimes c .
$$

Assertion 60. The coaction map $\boldsymbol{\sigma}^{\left(r^{\prime}\right)}$ is a "right inverse" for the multiaction map $\overline{\boldsymbol{\rho}}^{\left(r^{\prime}\right)}$

$$
\overline{\boldsymbol{\rho}}^{\left(r^{\prime}\right)} \circ \boldsymbol{\sigma}^{\left(r^{\prime}\right)}=\operatorname{id}_{C} .
$$

Proof. This follows from the normalization (8), (122).

Remark 61. The maps (121) and (122) establish the isomorphism $\overbrace{\mathbb{k} \otimes \ldots \otimes \mathbb{k}}^{r^{\prime}} \otimes C \cong C$, which is well-known in the binary case (see, e.g. [23]).

We can provide the definition of counit only in the case where the underlying field $\mathbb{k}$ has a unit.

Definition 62 (Counit axiom). The polyadic coalgebra $C^{(n)}(41)$ over the unital polyadic field $\mathbb{k}^{\left(m_{k}, n_{k}\right)}$ contains a $\mathbb{k}$-linear polyadic (right) counit map $\varepsilon^{\left(n^{\prime}, r^{\prime}\right)}: C^{\otimes\left(n^{\prime}-1\right)} \rightarrow K^{\otimes r^{\prime}}$ satisfying

$$
\left(\varepsilon^{\left(n^{\prime}, r^{\prime}\right)} \otimes \operatorname{id}_{C}\right) \circ \Delta^{\left(n^{\prime}\right)}=\sigma^{\left(r^{\prime}\right)},
$$

such that the diagram

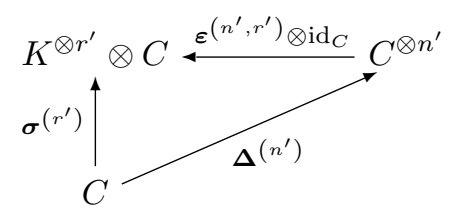

commutes (cf.(49)).

Remark 63. We cannot write the "elementwise" normalization action for the counit analogous to (50) (and state the Assertion 21), because a unit element in a (polyadic) coalgebra is not defined.

By analogy with the derived polyadic unit (see (52)), consider a "derived" version of the polyadic counit. 
Definition 64. The $\mathbb{k}$-linear derived polyadic counit (neutral counit sequence) of the polyadic coalgebra $C^{\left(n^{\prime}\right)}$ is the set $\hat{\varepsilon}^{(r)}=\left\{\varepsilon_{i}^{\left(r^{\prime}\right)}\right\}$ of $n^{\prime}-1$ maps $\varepsilon_{i}^{\left(r^{\prime}\right)}: C \rightarrow K^{\otimes r^{\prime}}, i=1, \ldots, n^{\prime}-1$, satisfying

$$
\left(\varepsilon_{1}^{\left(r^{\prime}\right)} \otimes \ldots \otimes \varepsilon_{n^{\prime}-1}^{\left(r^{\prime}\right)} \otimes \operatorname{id}_{C}\right) \circ \Delta^{\left(n^{\prime}\right)}=\sigma^{\left(r^{\prime}\right)},
$$

where $\operatorname{id}_{C}$ can be on any place. If $\varepsilon_{1}^{\left(r^{\prime}\right)}=\ldots=\varepsilon_{n^{\prime}-1}^{\left(r^{\prime}\right)}=\varepsilon_{0}^{\left(r^{\prime}\right)}$, we call it the strong derived polyadic counit. In general, we can define formally, cf. (53),

$$
\varepsilon_{d e r}^{\left(n^{\prime}, r^{\prime}\right)}=\varepsilon_{1}^{\left(r^{\prime}\right)} \otimes \ldots \otimes \varepsilon_{n^{\prime}-1}^{\left(r^{\prime}\right)} .
$$

Definition 65. A polyadic coassociative coalgebra $C_{d e r}^{\left(n^{\prime}\right)}=\left\langle C_{v e c t} \mid \boldsymbol{\Delta}_{d e r}^{\left(n^{\prime}\right)}, \boldsymbol{\varepsilon}_{d e r}^{\left(n^{\prime}, r^{\prime}\right)}\right\rangle$ is called derived from $C_{0}^{\left(n^{\prime}\right)}=\left\langle C_{\text {vect }} \mid \boldsymbol{\Delta}_{0}^{\left(n_{0}^{\prime}\right)}, \varepsilon_{0}^{\left(n_{0}^{\prime}, r^{\prime}\right)}\right\rangle$, if $(116)$ and

$$
\varepsilon_{d e r}^{\left(n^{\prime}, r^{\prime}\right)}=\overbrace{\varepsilon_{0}^{\left(n_{0}^{\prime}, r^{\prime}\right)} \otimes \ldots \otimes \varepsilon_{0}^{\left(n_{0}^{\prime}, r^{\prime}\right)}}^{\ell_{d}}
$$

hold, where $\varepsilon_{0}^{\left(n_{0}^{\prime}, r^{\prime}\right)}=\overbrace{\varepsilon_{0}^{\left(r^{\prime}\right)} \otimes \ldots \otimes \varepsilon_{0}^{\left(r^{\prime}\right)}}^{n_{0}^{\prime}-1}$ (formally, because id ${ }_{C}$ in (126) can be on any place).

In $[24,25]$ the particular case for $n^{\prime}=3$ and $r^{\prime}=1$ was considered.

\section{Homomorphisms of polyadic coalgebras}

In the binary case, a morphism of coalgebras is a linear map $\psi: C_{1} \rightarrow C_{2}$ which "commutes" with comultiplications (" $(\psi \otimes \psi) \circ \boldsymbol{\Delta}_{1}=\boldsymbol{\Delta}_{2} \circ \varphi$ "). It seems that for the polyadic coalgebras, one could formally change the direction of all arrows in (84). However, we observed that arity changing is possible for multivalued morphisms only. Therefore, here we confine ourselves to homomorphisms (1-place heteromorphisms [14]).

Let us consider two polyadic (equiary) $\mathbb{k}$-coalgebras $C_{1}^{\left(n^{\prime}\right)}=\left\langle C_{1} \mid \boldsymbol{\Delta}_{1}^{\left(n^{\prime}\right)}\right\rangle$ and $C_{2}^{\left(n^{\prime}\right)}=\left\langle C_{2} \mid \boldsymbol{\Delta}_{2}^{\left(n^{\prime}\right)}\right\rangle$ over the same polyadic field $\mathbb{k}^{\left(m_{k}, n_{k}\right)}$.

Definition 66. A (coalgebra) homomorphism between polyadic (equiary) coalgebras $C_{1}^{\left(n^{\prime}\right)}$ and $C_{2}^{\left(n^{\prime}\right)}$ is a $\mathbb{k}$ linear map $\Psi^{\left(n^{\prime}\right)}: C_{1} \rightarrow C_{2}$, such that

$$
(\overbrace{\boldsymbol{\Psi}^{\left(n^{\prime}\right)} \otimes \ldots \otimes \boldsymbol{\Psi}^{\left(n^{\prime}\right)}}^{n^{\prime}}) \circ \Delta_{1}^{\left(n^{\prime}\right)}=\Delta_{2}^{\left(n^{\prime}\right)} \circ \boldsymbol{\Psi}^{\left(n^{\prime}\right)}
$$

and the diagram

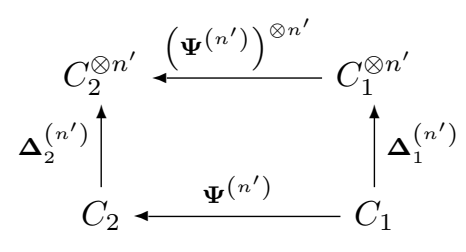

commutes (cf. (84)).

Only when the underlying field $\mathbb{k}$ is unital, we can also define a morphism for counits.

Definition 67. The counit homomorphism for $\varepsilon_{1,2}^{\left(n^{\prime}, r^{\prime}\right)}: C_{1,2}^{\otimes\left(n^{\prime}-1\right)} \rightarrow K^{\otimes r^{\prime}}$ is given by

$$
\varepsilon_{2}^{\left(n^{\prime}, r^{\prime}\right)}=\varepsilon_{1}^{\left(n^{\prime}, r^{\prime}\right)} \circ(\overbrace{\boldsymbol{\Psi}^{\left(n^{\prime}\right)} \otimes \ldots \otimes \boldsymbol{\Psi}^{\left(n^{\prime}\right)}}^{n^{\prime}-1}),
$$


and the diagram

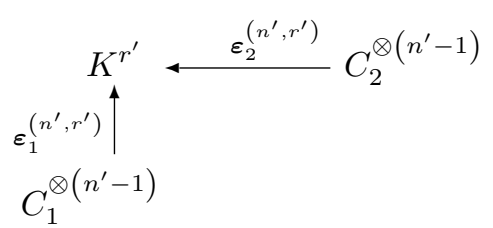

commutes (cf. (89)).

\section{Tensor product of polyadic coalgebras}

Let us consider $n^{\prime}$ polyadic equiary coalgebras $C_{i}^{\left(n^{\prime}\right)}=\left\langle C_{i} \mid \boldsymbol{\Delta}_{i}^{\left(n^{\prime}\right)}\right\rangle, i=1, \ldots, n^{\prime}$.

Proposition 68. The tensor product of the coalgebras has a structure of the polyadic coassociative coalgebra $C_{\otimes}^{\left(n^{\prime}\right)}=\left\langle C_{\otimes} \mid \Delta_{\otimes}^{\left(n^{\prime}\right)}\right\rangle, C_{\otimes}=\bigotimes_{i=1}^{n^{\prime}} C_{i}$, if

$$
\boldsymbol{\Delta}_{\otimes}^{\left(n^{\prime}\right)}=\boldsymbol{\tau}_{\text {medial }}^{\left(n^{\prime}, n^{\prime}\right)} \circ\left(\boldsymbol{\Delta}_{i}^{\left(n^{\prime}\right)} \otimes \ldots \otimes \boldsymbol{\Delta}_{i}^{\left(n^{\prime}\right)}\right)
$$

where $\boldsymbol{\tau}_{\text {medial }}^{\left(n^{\prime}, n^{\prime}\right)}$ is defined in $(69)$ and $\boldsymbol{\Delta}_{\otimes}^{\left(n^{\prime}\right)}: C_{\otimes} \rightarrow \overbrace{C_{\otimes} \otimes \ldots \otimes C_{\otimes}}^{n^{\prime}}$.

The proof is in full analogy with that of Proposition 36. If all of the coalgebras $C_{i}^{\left(n^{\prime}\right)}$ have counits, we denote them $\varepsilon_{i}^{\left(n^{\prime}, r^{\prime}\right)}: C_{i}^{\otimes\left(n^{\prime}-1\right)} \rightarrow K^{\otimes r^{\prime}}, i=1, \ldots, n^{\prime}$, and the counit map of $C_{\otimes}^{\left(n^{\prime}\right)}$ will be denoted by $\varepsilon_{\otimes}^{\left(n^{\prime}, r^{\prime}\right)}: C_{\otimes}^{\otimes\left(n^{\prime}-1\right)} \rightarrow K^{\otimes r^{\prime}}$. We have (in analogy to “ $\varepsilon_{C_{1} \otimes C_{2}}\left(c_{1} \otimes c_{2}\right)=\varepsilon_{C_{1}}\left(c_{1}\right) \varepsilon_{C_{2}}\left(c_{2}\right)$ ”)

Proposition 69. The tensor product coalgebra $C_{\otimes}^{\left(n^{\prime}\right)}$ has a counit which is defined by

$$
\begin{aligned}
& \varepsilon_{\otimes}^{\left(n^{\prime}, r^{\prime}\right)} \circ\left(c_{1} \otimes \ldots \otimes c_{n^{\prime}\left(n^{\prime}-1\right)}\right) \\
& =\boldsymbol{\mu}_{k}^{n_{k}} \circ\left(\varepsilon_{1}^{\left(n^{\prime}, r^{\prime}\right)} \circ\left(c_{1} \otimes \ldots \otimes c_{\left(n^{\prime}-1\right)}\right) \otimes \ldots \otimes \varepsilon_{n^{\prime}}^{\left(n^{\prime}, r^{\prime}\right)} \circ\left(c_{\left(n^{\prime}-1\right)\left(n^{\prime}-1\right)} \otimes \ldots \otimes c_{n^{\prime}\left(n^{\prime}-1\right)}\right)\right), \\
& c_{i} \in C_{i}, \quad i=1, \ldots, n^{\prime}\left(n^{\prime}-1\right),
\end{aligned}
$$

and the arity of the comultiplication coincides with the arity of the underlying field

$$
n^{\prime}=n_{k}
$$

\section{Polyadic coalgebras in the Sweedler notation}

The $\mathbb{k}$-linear coalgebra comultiplication map $\Delta^{\left(n^{\prime}\right)}$ defined in Definition 51 is useful for a "diagrammatic" description of polyadic coalgebras, and it corresponds to the algebra multiplication map $\boldsymbol{\mu}^{(n)}$, which both manipulate with sets. However, for concrete computations (with elements) we need an analog of the polyadic algebra multiplication $\mu^{(n)} \equiv \mu_{A}^{\left(n_{a}\right)}$ from (12). The connection of $\boldsymbol{\mu}^{(n)}$ and $\mu^{(n)}$ is given by (40), which can be treated as a "bridge" between the "diagrammatic" and "elementwise" descriptions. The co-analog of (40) was not considered, because the comultiplication has only one argument. To be consistent, we introduce the "elementwise" comultiplication $\Delta^{\left(n^{\prime}\right)}$ as the coanalog of $\mu^{(n)}$ by the evaluation

$$
\Delta^{\left(n^{\prime}\right)} \circ(c)=\Delta^{\left(n^{\prime}\right)}(c), \quad c \in C .
$$

In general, one does not distinguish $\boldsymbol{\Delta}^{\left(n^{\prime}\right)}$ and $\Delta^{\left(n^{\prime}\right)}$ and may use one symbol in both descriptions.

In real "elementwise" coalgebra computations with many variables and comultiplications acting on them, the indices and various letters reproduce themselves in such a way that it is impossible to observe the structure of the expressions. Therefore, instead of different letters in the binary decomposition (" $\Delta(c)=\sum_{i} a_{i} \otimes b_{i}$ " and (104)) it was proposed [32] to use the same letter (" $\Delta(c)=\sum_{i} c_{[1], i} \otimes c_{[2], i}$ "), and then go from the real sum $\sum_{i}$ to the formal sum $\sum_{[c]}$ as (" $\Delta(c)=\sum_{[c]} c_{[1]} \otimes c_{[2]}$ " remembering the place of the components $c_{[1]}, c_{[2]}$ only), because the real indices pullulate in complicated formulas enormously. In simple cases, the sum sign was also omitted (" $\Delta(c)=c_{[1]} \otimes c_{[2]}$ "), which recalls the Einstein index summation rule in physics. This trick abbreviated 
tedious coalgebra computations and was called the (sumless) Sweedler (sigma) notation (sometimes it is called the Heyneman-Sweedler notation [33]).

Now we can write $\Delta^{\left(n^{\prime}\right)}$ as a $n^{\prime}$-ary decomposition in the manifest "elementwise" form

$$
\Delta^{\left(n^{\prime}\right)}(c)=\left(\nu^{(m)}\right)^{\circ \ell_{\Delta}}\left[c_{[1], 1} \otimes c_{[2], 1} \otimes \ldots \otimes c_{\left[n^{\prime}\right], 1}, \ldots, c_{[1], N_{\Delta}} \otimes c_{[2], N_{\Delta}} \otimes \ldots \otimes c_{\left[n^{\prime}\right], N_{\Delta}}\right], \quad c_{[j], i} \in C,
$$

where $\ell_{\Delta} \in \mathbb{N}_{0}$ is a "number of additions", and $N_{\Delta} \in \mathbb{N}$ is the "number of summands". In the binary case, the number of summands in the decomposition is not "algebraically" restricted, because $N_{\Delta}=\ell_{\Delta}+1$. In the polyadic case, we have

Assertion 70. The admissible "number of summands" $N_{\Delta}$ in the polyadic comultiplication is

$$
N_{\Delta}=\ell_{\Delta}(m-1)+1, \quad \ell_{\Delta} \in \mathbb{N}_{0}, \quad m \geq 2 .
$$

Therefore, the "quantization" of $N_{\Delta}$ coincides with that of the $N$-dimensional polyadic algebra (see Assertion 44).

Introduce the polyadic Sweedler notation by exchanging in (137) the real $m$-ary addition $\nu^{(m)}$ by the formal addition $\nu_{[c]}$ and writing

$$
\Delta^{\left(n^{\prime}\right)}(c)=\nu_{[c]}\left[c_{[1]} \otimes c_{[2]} \otimes \ldots \otimes c_{\left[n^{\prime}\right]}\right] \Rightarrow c_{[1]} \otimes c_{[2]} \otimes \ldots \otimes c_{\left[n^{\prime}\right]} .
$$

Remember here that we can formally add only $N_{\Delta}$ summands, because of the "quantization" (138) rule.

The polyadic Sweedler notation power can be seen in the following

Example 71. We apply (139) to the coassociativity (111) with $n^{\prime}=3$, to obtain

$$
\begin{aligned}
\left(\mathrm{id} \otimes \mathrm{id} \otimes \Delta^{(3)}\right) \circ \Delta^{(3)}(c) & =\left(\mathrm{id} \otimes \Delta^{(3)} \otimes \mathrm{id}\right) \circ \Delta^{(3)}(c)=\left(\Delta^{(3)} \otimes \mathrm{id} \otimes \mathrm{id}\right) \circ \Delta^{(3)}(c) \Rightarrow \\
& =\nu_{[c]}\left[c_{[1]} \otimes c_{[2]} \otimes \nu_{\left[c_{2}\right]}\left[\left(c_{[3]}\right)_{[1]} \otimes\left(c_{[3]}\right)_{[2]} \otimes\left(c_{[3]}\right)_{[3]}\right]\right] \\
& =\nu_{[c]}\left[c_{[1]} \otimes \nu_{\left[c_{2}\right]}\left[\left(c_{[2]}\right)_{[1]} \otimes\left(c_{[2]}\right)_{[2]} \otimes\left(c_{[2]}\right)_{[3]}\right] \otimes c_{[3]}\right] \\
& =\nu_{[c]}\left[\nu_{\left[c_{2}\right]}\left[\left(c_{[1]}\right)_{[1]} \otimes\left(c_{[1]}\right)_{[2]} \otimes\left(c_{[1]}\right)_{[3]}\right] \otimes c_{[2]} \otimes c_{[3]}\right] .
\end{aligned}
$$

After dropping the brackets and applying the Sweedler trick for the second time, we get the same formal expression in all three cases

$$
\left(\nu_{[c]}\right)^{\circ 2}\left[c_{[1]} \otimes c_{[2]} \otimes c_{[3]} \otimes c_{[4]} \otimes c_{[5]}\right] .
$$

Unfortunately, in the polyadic case the Sweedler notation looses too much information to be useful.

Assertion 72. The polyadic Sweedler notation can be applied to only the derived polyadic coalgebras.

Nevertheless, if in an expression there are no coiterations, one can formally use it (e.g., in the polyadic analog (124) of the counting axiom " $\left.\sum \varepsilon\left(c_{[1]}\right) c_{[2]}=c "\right)$.

\section{Polyadic group-like and primitive elements}

Let us consider some special kinds of elements in a polyadic coalgebra $C^{\left(n^{\prime}\right)}$. We should take into account that in the polyadic case, as in (137), there can only be the admissible "number of summands" $N_{\Delta}(138)$.

Definition 73. An element $g$ of $C^{\left(n^{\prime}\right)}$ is called polyadic semigroup-like, if

$$
\Delta^{\left(n^{\prime}\right)}(g)=\overbrace{g \otimes \ldots \otimes g}^{n^{\prime}},, \quad g \in C .
$$

When $C^{\left(n^{\prime}\right)}$ has the counit $\varepsilon^{\left(n^{\prime}, r^{\prime}\right)}(124)$, then $g$ is called polyadic group-like, if (" $\varepsilon(g)=1$ ”)

$$
\varepsilon^{\left(n^{\prime}, r^{\prime}\right)} \circ(\overbrace{g \otimes \ldots \otimes g}^{n^{\prime}-1})=\overbrace{e_{k} \otimes \ldots \otimes e_{k}}^{r^{\prime}},
$$

where $e_{k}$ is the unit of the underlying polyadic field $\mathbb{k}$. 
Definition 74. An element $x$ of $C^{\left(n^{\prime}\right)}$ is called polyadic skew $k_{p}$-primitive, if (" $\Delta(x)=g_{1} \otimes x+x \otimes g_{2}$ ")

$$
\begin{aligned}
\Delta^{\left(n^{\prime}\right)}(x) & =\left(\nu^{(m)}\right)^{\circ \ell_{\Delta}}\left[(\overbrace{g_{1} \otimes \ldots \otimes g_{k_{p}}}^{k_{p}} \otimes \overbrace{x \otimes \ldots \otimes x}^{n^{\prime}-k_{p}}), \ldots,\right. \\
& \left.(\overbrace{x \otimes \ldots \otimes x}^{n^{\prime}-k_{p}} \otimes \overbrace{g_{\left(N_{\Delta}-1\right) k_{p}+1} \otimes \ldots \otimes g_{N_{\Delta} k_{p}}}^{k_{p}})\right],
\end{aligned}
$$

where $1 \leq k_{p} \leq n^{\prime}-1, N_{\Delta}=\ell_{\Delta}(m-1)+1$ is the total "number of summands", here $\ell_{\Delta} \in \mathbb{N}$ is the "number of $m$-ary additions", and $g_{i} \in C, i=1, \ldots, N_{\Delta} k_{p}$ are polyadic (semi-)group-like (143). In (145) the $n^{\prime}-k_{p}$ elements $x$ move from the right to the left by one.

Assertion 75. If $k_{p}=n^{\prime}-1$, then $\Delta^{\left(n^{\prime}\right)}(x)$ is "linear" in $x$, and $n^{\prime}=\ell_{\Delta}(m-1)+1$.

In this case, we call $x$ a polyadic primitive element.

Example 76. Let $n^{\prime}=3$ and $k_{p}=2$, then $m=3$, and we have only one ternary addition $\ell_{\Delta}=1$

$$
\begin{aligned}
& \Delta^{(3)}(x)=\nu^{(3)}\left[g_{1} \otimes g_{2} \otimes x, g_{3} \otimes x \otimes g_{4}, x \otimes g_{5} \otimes g_{6}\right], \\
& \Delta^{(3)}\left(g_{i}\right)=g_{i} \otimes g_{i} \otimes g_{i}, \quad i=1, \ldots, 6 .
\end{aligned}
$$

The ternary coassociativity gives $g_{1}=g_{2}=g_{3}$ and $g_{4}=g_{5}=g_{6}$. Therefore, the general form of the ternary primitive element is

$$
\Delta^{(3)}(x)=\nu^{(3)}\left[g_{1} \otimes g_{1} \otimes x, g_{1} \otimes x \otimes g_{2}, x \otimes g_{2} \otimes g_{2}\right] .
$$

Note that coassociativity leads to the derived comultiplication (116), because

$$
\begin{aligned}
& \Delta^{(3)}(x)=\left(\mathrm{id} \otimes \Delta^{(2)}\right) \Delta^{(2)}(x)=\left(\Delta^{(2)} \otimes \mathrm{id}\right) \Delta^{(2)}(x), \\
& \Delta^{(2)}(x)=g_{1} \otimes x+x \otimes g_{2} .
\end{aligned}
$$

The same situation occurs with the "linear" comultiplication of any arity $n^{\prime}$, i.e. when $k_{p}=n^{\prime}-1$.

The most important difference with the binary case is the "intermediate" possibility $k_{p}<n^{\prime}-1$, when the r.h.s. is "nonlinear" in $x$.

Example 77. In the case where $n^{\prime}=3$ and $k_{p}=1$, we have $m=3$, and $\ell_{\Delta}=1$

$$
\begin{aligned}
& \Delta^{(3)}(x)=\nu^{(3)}\left[g_{1} \otimes x \otimes x, x \otimes g_{2} \otimes x, x \otimes x \otimes g_{3}\right], \\
& \Delta^{(3)}\left(g_{i}\right)=g_{i} \otimes g_{i} \otimes g_{i}, \quad i=1, \ldots, 3 .
\end{aligned}
$$

Now ternary coassociativity cannot be achieved with any values of $g_{i}$. This is true for any arity $n^{\prime}$ and any "nonlinear" comultiplication.

Therefore, we arrive at the general structure

Assertion 78. In a polyadic coassociative coalgebra $C^{\left(n^{\prime}\right)}$ polyadic primitive elements exist, if and only if the $n^{\prime}$-ary comultiplication $\Delta^{\left(n^{\prime}\right)}$ is derived (116) from the binary comultiplication $\Delta^{(2)}$.

\section{Polyadic analog of duality}

The connection between binary associative algebras and coassociative coalgebras (formally named as "reversing arrows") is given in terms of the dual vector space (dual module) concept. Informally, for a binary coalgebra $C^{(2)}=\langle C \mid \Delta, \varepsilon\rangle$ considered as a vector space over a binary field $k$ (a $k$-vector space), its $d u a l$ is $C_{2}^{*}=\operatorname{Hom}_{k}(C, k)$ with the natural pairing $C^{*} \times C \rightarrow k$ given by $f(c), f \in C^{*}, c \in C$. The canonical injection $\theta: C^{*} \otimes C^{*} \rightarrow(C \otimes C)^{*}$ is defined by

$$
\theta\left(f_{1} \otimes f_{2}\right) \circ\left(c_{1} \otimes c_{2}\right)=f_{1}\left(c_{1}\right) f_{2}\left(c_{2}\right), \quad c_{1,2} \in C, \quad f_{1,2} \in C^{*},
$$

which is an isomorphism in the finite-dimensional case. The transpose of $\Delta: C \rightarrow C \otimes C$ is a $k$-linear map $\Delta_{*}:(C \otimes C)^{*} \rightarrow C^{*}$ acting as $\Delta_{*}(\xi)(c)=\xi \circ(\Delta(c))$, where $\xi \in(C \otimes C)^{*}, c \in C$. The multiplication $\mu_{*}$ on the set $C^{*}$ is the map $C^{*} \otimes C^{*} \rightarrow C^{*}$, and therefore we have to use the canonical injection $\theta$ as follows

$$
\begin{aligned}
& \mu_{*}: C^{*} \otimes C^{*} \stackrel{\theta}{\rightarrow}(C \otimes C)^{*} \stackrel{\Delta_{*}}{\rightarrow} C^{*}, \\
& \mu_{*}=\Delta_{*} \circ \theta .
\end{aligned}
$$


The associativity of $\mu_{*}$ follows from the coassociativity of $\Delta$. Since $k^{*} \simeq k$, the dual of the counit is the unit $\eta_{*}: k \stackrel{\varepsilon^{*}}{\rightarrow} C^{*}$. Therefore, $C^{(2) *}=\left\langle C^{*} \mid \mu_{*}, \eta_{*}\right\rangle$ is a binary associative algebra which is called the dual algebra of the binary coalgebra $C^{(2)}=\langle C \mid \Delta, \varepsilon\rangle$ (see, e.g. [3]).

In the polyadic case, arities of the comultiplication, its dual multiplication and the underlying field can be different, but connected by (153). Let us consider a polyadic coassociative coalgebra $C^{\left(n^{\prime}\right)}$ with $n^{\prime}$-ary comultiplication $\Delta^{\left(n^{\prime}\right)}(136)$ over $\mathbb{k}^{\left(m_{k}, n_{k}\right)}$. In search of the most general polyadic analog of the injection (153), we arrive at the possibility of multiplace morphisms.

Definition 79. For the polyadic coalgebra $C^{\left(n^{\prime}\right)}$ considered as a polyadic vector space over $\mathbb{k}^{\left(m_{k}, n_{k}\right)}$, a polyadic dual is $C^{*}=\operatorname{Hom}_{\mathbb{k}}\left(C^{\otimes s}, K\right)$ with s-place pairing $C^{*} \times \overbrace{C \times \ldots \times C}^{s} \rightarrow K$ giving by $f^{(s)}\left(c_{1}, \ldots, c_{s}\right), f \in C^{*}$, $c_{i} \in C, s \in \mathbb{N}$.

While constructing a polyadic analog of (153), recall that for any $n^{\prime}$-ary operation the admissible length of a co-word is $\ell^{\prime}\left(n^{\prime}-1\right)+1$, where $\ell^{\prime}$ is the number of the iterated operation (115).

Definition 80. A polyadic canonical injection map $\boldsymbol{\theta}^{\left(n^{*}, n^{\prime}, s\right)}$ of $C^{\left(n^{\prime}\right)}$ is defined by

$$
\begin{aligned}
& \boldsymbol{\theta}^{\left(n^{*}, n^{\prime}, s\right)} \circ\left(f_{1}^{(s)} \otimes \ldots \otimes f_{n^{*}}^{(s)}\right) \circ\left(c_{1} \otimes \ldots \otimes c_{\ell^{\prime}\left(n^{\prime}-1\right)+1}\right)= \\
& \left(\mu_{k}^{\left(n_{k}\right)}\right)^{\circ \ell_{k}}\left[f_{1}^{(s)}\left(c_{1}, \ldots, c_{s}\right), \ldots, f_{\ell_{k}\left(n_{k}-1\right)+1}^{(s)}\left(c_{\left(n^{*}-1\right) s+1}, \ldots, c_{n^{*} s}\right)\right],
\end{aligned}
$$

where

$$
\begin{aligned}
n^{*} s & =\ell^{\prime}\left(n^{\prime}-1\right)+1, \quad \ell^{\prime} \in \mathbb{N}, \quad n^{\prime} \geq 2, \\
n^{*} & =\ell_{k}\left(n_{k}-1\right)+1, \quad \ell_{k} \in \mathbb{N}, \quad n_{k} \geq 2 .
\end{aligned}
$$

It is obvious that $\theta^{(2,2,1)}=\theta$ from (153). Then, the polyadic transpose map of the $n^{\prime}$-ary comultiplication $\Delta^{\left(n^{\prime}\right)}: C \rightarrow \overbrace{C \otimes \ldots \otimes C}^{n^{\prime}}$ is a $\mathbb{k}$-linear map $\Delta_{*}^{\left(n^{\prime \prime}\right)}:(\overbrace{C \otimes \ldots \otimes C}^{n^{\prime \prime}})^{*} \rightarrow C^{*}$ such that

$$
\begin{aligned}
& \Delta_{*}^{\left(n^{\prime \prime}\right)} \circ\left(\xi^{\left(n^{\prime \prime}\right)}\right)(c)=\xi^{\left(n^{\prime \prime}\right)} \circ\left(\left(\Delta^{\left(n^{\prime}\right)}\right)^{\circ \ell^{\prime}}(c)\right), \\
& \xi^{\left(n^{\prime \prime}\right)} \in(\overbrace{C \otimes \ldots \otimes C}^{n^{\prime \prime}})^{*}, n^{\prime \prime}=\ell^{\prime}\left(n^{\prime}-1\right)+1, c \in C
\end{aligned}
$$

where $\ell^{\prime}$ is the "number of comultiplications" (see (6) for multiplications and (115)).

Definition 81. A $n^{*}$-ary multiplication map $\boldsymbol{\mu}_{*}^{\left(n^{*}\right)}$ which is (one way) dual to the $n^{\prime}$-ary comultiplication map $\boldsymbol{\Delta}^{\left(n^{\prime}\right)}$ is given by the composition of the polyadic canonical injection $\boldsymbol{\theta}^{\left(n^{*}, n^{\prime}, s\right)}(156)$ and the polyadic transpose $\boldsymbol{\Delta}_{*}^{\left(n^{\prime \prime}\right)}(159)$ by

$$
\boldsymbol{\mu}_{*}^{\left(n^{*}\right)}=\boldsymbol{\Delta}_{*}^{\left(n^{\prime \prime}\right)} \circ \boldsymbol{\theta}^{\left(n^{*}, n^{\prime}, s\right)} .
$$

Indeed, using (156) and (159) we obtain (in Sweedler notation)

$$
\begin{aligned}
& \boldsymbol{\mu}_{*}^{\left(n^{*}\right)} \circ\left(f_{1}^{(s)} \otimes \ldots \otimes f_{n^{*}}^{(s)}\right) \circ(c)=\boldsymbol{\Delta}_{*}^{\left(n^{\prime \prime}\right)} \circ \boldsymbol{\theta}^{\left(n^{*}, n^{\prime}, s\right)} \circ\left(f_{1}^{(s)} \otimes \ldots \otimes f_{n^{*}}^{(s)}\right) \circ(c) \\
& =\boldsymbol{\theta}^{\left(n^{*}, n^{\prime}, s\right)} \circ\left(f_{1}^{(s)} \otimes \ldots \otimes f_{n^{*}}^{(s)}\right) \circ\left(\left(\Delta^{\left(n^{\prime}\right)}\right)^{\circ \ell^{\prime}}(c)\right) \\
& =\left(\mu_{k}^{\left(n_{k}\right)}\right)^{\circ \ell_{k}}\left[f_{1}^{(s)}\left(c_{[1]}, \ldots, c_{[s]}\right), \ldots, f_{\ell_{k}\left(n_{k}-1\right)+1}^{(s)}\left(c_{\left[\left(n^{*}-1\right) s+1\right]}, \ldots, c_{\left[n^{*} s\right]}\right)\right],
\end{aligned}
$$

and (157)-(158) are valid, from which we arrive at

Assertion 82. In the polyadic case the arity $n^{*}$ of the multiplication $\boldsymbol{\mu}_{*}^{\left(n^{*}\right)}$ can be different from the arity $n^{\prime}$ of the initial coalgebra $C^{\left(n^{\prime}\right)}$. 
Remark 83. If $n^{*} \neq n^{\prime}$ and $s \geq 2$, the word "duality" can only be used conditionally.

\section{Polyadic convolution product}

If $A^{(2)}=\langle A \mid \mu, \eta\rangle$ is a binary algebra and $C^{(2)}=\langle C \mid \Delta, \varepsilon\rangle$ is a binary coalgebra over a binary field $k$, then a more general set of $k$-linear maps $\operatorname{Hom}_{k}(C, A)$ can be considered, while its particular case where $A^{(2)}=k$ corresponds to the above duality. The multiplication on $\operatorname{Hom}_{k}(C, A)$ is the convolution product $(\star)$ which can be uniquely constructed in the natural way: by applying first comultiplication $\Delta$ and then multiplication $\mu \equiv(\cdot)$ to an element of $C$, as $C \stackrel{\Delta}{\longrightarrow} C \otimes C \stackrel{f \otimes g}{\longrightarrow} A \otimes A \stackrel{\mu}{\longrightarrow} A$ or $f \star g=\mu \circ(f \otimes g) \circ \Delta$, where $f, g \in \operatorname{Hom}_{k}(C, A)$. The associativity of the convolution product follows from the associativity of $\mu$ and coassociativity of $\Delta$, and the role of the identity (neutral element) in $\operatorname{Hom}_{k}(C, A)$ is played by the composition of the unit map $\eta: k \rightarrow A$ and the counit map $\varepsilon: C \rightarrow k$, such that $e_{\star}=\eta \circ \varepsilon \in \operatorname{Hom}_{k}(C, A)$, because $e_{\star} \star f=f \star e_{\star}=f$. Indeed, from the obvious relation $\operatorname{id}_{A} \circ f \circ \operatorname{id}_{C}=f$ and the unit and counit axioms it follows that

$$
\mu \circ\left(\eta \otimes \operatorname{id}_{A}\right) \circ\left(\operatorname{id}_{K} \otimes f\right) \circ\left(\varepsilon \otimes \operatorname{id}_{C}\right) \circ \Delta=\mu \circ\left(\eta \circ \operatorname{id}_{K} \circ \varepsilon\right) \otimes\left(\operatorname{id}_{A} \circ f \circ \operatorname{id}_{C}\right) \circ \Delta=e_{\star} \star f=f,
$$

or in Sweedler notation $\varepsilon\left(c_{[1]}\right) \cdot f\left(c_{[2]}\right)=f\left(c_{[1]}\right) \cdot \varepsilon\left(c_{[2]}\right)=f(c)$.

The polyadic analog of duality and (161) offer an idea of how to generalize the binary convolution product to the most exotic case, when the algebra and coalgebra have different arities $n \neq n^{\prime}$.

Let $A^{(n)}$ and $C^{\left(n^{\prime}\right)}$ be, respectively, a polyadic associative algebra and a coassociative coalgebra over the same polyadic field $\mathbb{k}^{\left(m_{k}, n_{k}\right)}$. If they are both unital and counital respectively, then we can consider a polyadic analog of the composition $\eta \circ \varepsilon$. The crucial difference from the binary case is that now $\boldsymbol{\eta}^{(r, n)}$ and $\boldsymbol{\varepsilon}^{\left(n^{\prime}, r^{\prime}\right)}$ are multiplace multivalued maps (48) and (124). Their composition is

$$
\boldsymbol{e}_{\star}^{\left(n^{\prime}, n\right)}=\boldsymbol{\eta}^{(r, n)} \circ \boldsymbol{\gamma}^{\left(r^{\prime}, r\right)} \circ \boldsymbol{\varepsilon}^{\left(n^{\prime}, r^{\prime}\right)} \in \operatorname{Hom}_{\mathbb{k}}\left(C^{\otimes\left(n^{\prime}-1\right)}, A^{\otimes(n-1)}\right),
$$

where the multiplace multivalued map $\gamma^{\left(r^{\prime}, r\right)} \in \operatorname{Hom}_{\mathbb{k}}\left(K^{\otimes r^{\prime}}, K^{\otimes r}\right)$ is, obviously, $(\simeq)$, and the diagram

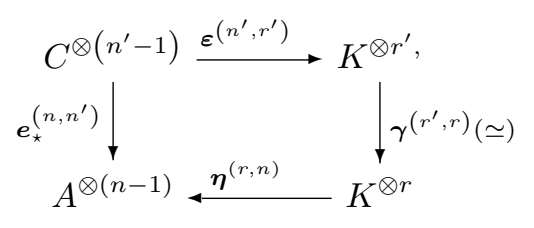

commutes.

The formula (163) leads us to propose

Conjecture 84. A polyadic analog of the convolution should be considered for multiplace multivalued $\mathbb{k}$-linear maps in $\operatorname{Hom}_{\mathbb{k}}\left(C^{\otimes\left(n^{\prime}-1\right)}, A^{\otimes(n-1)}\right)$.

In this way, we arrive at the following

Construction 85. Introduce the $\mathbb{k}$-linear maps $\mathbf{f}^{(i)}: C^{\otimes\left(n^{\prime}-1\right)} \rightarrow A^{\otimes(n-1)}, i=1, \ldots, n_{\star}$, where $n_{\star} \geq 2$. To create a closed $n_{\star}$-ary operation for them, we use the $\ell$-iterated multiplication map $\left(\boldsymbol{\mu}^{(n)}\right)^{\circ \ell}: A^{\otimes \ell(n-1)+1} \rightarrow A$ and $\ell^{\prime}$-iterated comultiplication map $\left(\Delta^{\left(n^{\prime}\right)}\right)^{\circ \ell^{\prime}}: C \rightarrow C^{\otimes \ell^{\prime}\left(n^{\prime}-1\right)+1}$. Then we compose the above $\mathbb{k}$-linear maps in the same way as is done above for the binary case

$$
\begin{aligned}
& C^{\otimes\left(n^{\prime}-1\right)} \stackrel{\left(\left(\Delta^{\left(n^{\prime}\right)}\right)^{\circ \ell^{\prime}}\right)^{\otimes\left(n^{\prime}-1\right)}}{\longrightarrow} C^{\otimes\left(n^{\prime}-1\right)\left(\ell^{\prime}\left(n^{\prime}-1\right)+1\right)} \stackrel{\stackrel{\boldsymbol{\tau}_{\text {medial }}^{\left(n_{\star}, n^{\prime}-1\right)} \longrightarrow}{\longrightarrow} C^{\otimes\left(n^{\prime}-1\right)\left(\ell^{\prime}\left(n^{\prime}-1\right)+1\right)}}{\stackrel{\mathbf{f}^{(1)} \otimes \ldots \otimes \mathbf{f}^{\left(n_{\star}\right)}}{\longrightarrow} A^{\otimes(n-1)\left(\ell^{\prime}\left(n^{\prime}-1\right)+1\right)} \stackrel{\boldsymbol{\tau}_{\text {medial }}^{\left(n-1, n_{\star}\right)}}{\longrightarrow} A^{\otimes(n-1)(\ell(n-1)+1)} \stackrel{\left(\left(\boldsymbol{\mu}^{(n)}\right)^{\circ \ell}\right)^{\otimes(n-1)} \longrightarrow}{\longrightarrow} A^{\otimes(n-1)} .}
\end{aligned}
$$

where $\boldsymbol{\tau}_{\text {medial }}^{\left(n_{\star}, n^{\prime}-1\right)}$ and $\boldsymbol{\tau}_{\text {medial }}^{\left(n-1, n_{\star}\right)}$ are the medial maps (69) acting on the Sweedler components of $c$ and $\mathbf{f}^{(i)}$, respectively. To make the sequence of maps (165) consistent, the arity $n_{\star}$ is connected with the iteration numbers $\ell, \ell^{\prime}$ by $n_{\star}=\ell(n-1)+1=\ell^{\prime}\left(n^{\prime}-1\right)+1, \ell, \ell^{\prime} \in \mathbb{N}$.

Definition 86. Let $A^{(n)}$ and $C^{\left(n^{\prime}\right)}$ be a $n$-ary associative algebra and $n^{\prime}$-ary coassociative coalgebra over a polyadic field $\mathbb{k}$ (the existence of the unit and counit here is mandatory), then the set $\operatorname{Hom}_{\mathbb{k}}\left(C^{\otimes\left(n^{\prime}-1\right)}, A^{\otimes(n-1)}\right)$ 
is closed under the $n_{\star}$-ary convolution product map $\boldsymbol{\mu}_{\star}^{\left(n_{\star}\right)}$ defined by

$$
\begin{aligned}
& \boldsymbol{\mu}_{\star}^{\left(n_{\star}\right)} \circ\left(\mathbf{f}^{(1)} \otimes \ldots \otimes \mathbf{f}^{\left(n_{\star}\right)}\right)= \\
& \left(\left(\boldsymbol{\mu}^{(n)}\right)^{\circ \ell}\right)^{\otimes(n-1)} \circ \boldsymbol{\tau}_{\text {medial }}^{\left(n-1, n_{\star}\right)} \circ\left(\mathbf{f}^{(1)} \otimes \ldots \otimes \mathbf{f}^{\left(n_{\star}\right)}\right) \circ \boldsymbol{\tau}_{\text {medial }}^{\left(n_{\star}, n^{\prime}-1\right)} \circ\left(\left(\boldsymbol{\Delta}^{\left(n^{\prime}\right)}\right)^{\circ \ell^{\prime}}\right)^{\otimes\left(n^{\prime}-1\right)},
\end{aligned}
$$

and its arity is given by the following $n_{\star}$-consistency condition

$$
n_{\star}-1=\ell(n-1)=\ell^{\prime}\left(n^{\prime}-1\right) .
$$

Definition 87. The set of $\mathbb{k}$-linear maps $\mathbf{f}^{(i)} \in \operatorname{Hom}_{\mathbb{k}}\left(C^{\otimes\left(n^{\prime}-1\right)}, A^{\otimes(n-1)}\right)$ endowed with the convolution product (166) is called a polyadic convolution algebra

$$
C_{\star}^{\left(n^{\prime}, n\right)}=\left\langle\operatorname{Hom}_{\mathbb{k}}\left(C^{\otimes\left(n^{\prime}-1\right)}, A^{\otimes(n-1)}\right) \mid \boldsymbol{\mu}_{\star}^{\left(n_{\star}\right)}\right\rangle .
$$

Example 88. An important case is given by the binary algebra $A^{(2)}$ and coalgebra $C^{(2)}\left(n=n^{\prime}=2\right)$, when the number of iterations are equal $\ell=\ell^{\prime}$, and the arity $n_{\star}$ becomes

$$
n_{\star}=\ell+1=\ell^{\prime}+1, \quad \ell, \ell^{\prime} \in \mathbb{N},
$$

while the $n_{\star}$-ary convolution product in $\operatorname{Hom}_{\mathbb{k}}(C, A)$ takes the form

$$
\boldsymbol{\mu}_{\star}^{\left(n_{\star}\right)} \circ\left(\mathbf{f}^{(1)} \otimes \ldots \otimes \mathbf{f}^{\left(n_{\star}\right)}\right)=\boldsymbol{\mu}^{\circ\left(n_{\star}-1\right)} \circ\left(\mathbf{f}^{(1)} \otimes \ldots \otimes \mathbf{f}^{\left(n_{\star}\right)}\right) \circ \boldsymbol{\Delta}^{\circ\left(n_{\star}-1\right)}, \quad \mathbf{f}^{(i)} \in \operatorname{Hom}_{\mathbb{k}}(C, A),
$$

where $\boldsymbol{\mu}=\boldsymbol{\mu}^{(2)}$ and $\boldsymbol{\Delta}=\boldsymbol{\Delta}^{(2)}$ are the binary multiplication and comultiplication maps respectively.

Definition 89. The polyadic convolution algebra $C_{\star}^{(2,2)}$ determined by the binary algebra and binary coalgebra (170) is called derived.

Corollary 90. The arity $n_{\star}$ of the derived polyadic convolution algebra is unrestricted and can take any integer value $n_{\star} \geq 2$.

Remark 91. If the polyadic tensor product and the underlying polyadic field $\mathbb{k}$ are derived (see discussion [10]), while all maps coincide $\mathbf{f}^{(i)}=\mathbf{f}$, the convolution product (170) is called the Sweedler power of $\mathbf{f}[34]$ or the Adams operator [35]. In the binary case they denoted it by $(\mathbf{f})^{n_{\star}}$, but for the $n_{\star}$-ary product this is the first polyadic power of $\mathbf{f}$ (see (6)).

Obviously, some interesting algebraic objects are nonderived, and here they are determined by $n+n^{\prime} \geq 5$, and also the arities of the algebra and coalgebra can be different $n \neq n^{\prime}$, which is a more exotic and exciting possibility. Generally, the arity $n_{\star}$ of the convolution product (166) is not arbitrary and is "quantized" by solving (167) in integers. The values $n_{\star}$ for minimal arities $n, n^{\prime}$ are presented in TABLE 3.

The most unusual possibility is the existence of nondiagonal entries, which correspond to unequal arities of multiplication and comultiplication $n \neq n^{\prime}$. The table is symmetric, which means that the arity $n_{\star}$ is invariant under the exchange $(n, \ell) \longleftrightarrow\left(n^{\prime}, \ell^{\prime}\right)$ following from (167).

Example $92\left(\operatorname{Hom}_{\mathbb{k}}(C, A)\right)$. In the simplest derived case (170), when both algebra $A^{(2)}=\langle A \mid \boldsymbol{\mu}\rangle$ and coalgebra $C^{(2)}=\langle C \mid \boldsymbol{\Delta}\rangle$ are binary with $n=2, \ell=2, n^{\prime}=2, \ell^{\prime}=2$, it is possible to obtain the ternary convolution product $\boldsymbol{\mu}_{\star}^{(3)}$ of the maps $\mathbf{f}^{(i)}: C \rightarrow A, i=1,2,3$, using Sweedler notation for $\boldsymbol{\Delta}^{\circ 2} \equiv\left(\operatorname{id}_{C} \otimes \boldsymbol{\Delta}\right) \circ \boldsymbol{\Delta}$ as $\Delta^{\circ 2}(c)=c_{[1]} \otimes c_{[2]} \otimes c_{[3]}, \boldsymbol{\mu}^{\circ 2} \equiv \boldsymbol{\mu} \circ\left(\mathrm{id}_{A} \otimes \boldsymbol{\mu}\right): A^{\otimes 3} \rightarrow A$, and the elementwise description using the evaluation

$$
\boldsymbol{\mu}_{\star, \text { der }}^{(3)} \circ\left(\mathbf{f}^{(1)} \otimes \mathbf{f}^{(2)} \otimes \mathbf{f}^{(3)}\right) \circ(c)=\mu^{\circ 2}\left[f^{(1)}\left(c_{[1]}\right), f^{(2)}\left(c_{[2]}\right), f^{(3)}\left(c_{[3]}\right)\right] .
$$

Example $93\left(\operatorname{Hom}_{\mathbb{k}}\left(C, A^{\otimes 2}\right), \operatorname{Hom}_{\mathbb{k}}\left(C^{\otimes 2}, A\right)\right)$. Nonbinary, nonderived and nonsymmetric cases:

1. The ternary algebra $A^{(3)}=\left\langle A \mid \boldsymbol{\mu}^{(3)}\right\rangle$ and the binary coalgebra $C^{(2)}=\langle C \mid \boldsymbol{\Delta}\rangle$, such that $n=3, \ell=1, n^{\prime}=$ $2, \ell^{\prime}=2$ giving a ternary convolution product of the maps $\mathbf{f}^{(i)}: C \rightarrow A^{\otimes 2}, i=1,2,3$. In the elementwise description $\mathbf{f}^{(i)} \circ(c)=f_{[1]}^{(i)}(c) \otimes f_{[2]}^{(i)}(c), c \in C$. Using (166), we obtain the manifest form of the nonderived ternary convolution product by evaluation

$$
\begin{aligned}
& \boldsymbol{\mu}_{\star}^{(3)} \circ\left(\mathbf{f}^{(1)} \otimes \mathbf{f}^{(2)} \otimes \mathbf{f}^{(3)}\right) \circ(c) \\
& =\mu^{(3)}\left[f_{[1]}^{(1)}\left(c_{[1]}\right), f_{[1]}^{(2)}\left(c_{[2]}\right), f_{[1]}^{(3)}\left(c_{[3]}\right)\right] \otimes \mu^{(3)}\left[f_{[2]}^{(1)}\left(c_{[1]}\right), f_{[2]}^{(2)}\left(c_{[2]}\right), f_{[2]}^{(3)}\left(c_{[3]}\right)\right],
\end{aligned}
$$

where $\Delta^{\circ 2}(c)=c_{[1]} \otimes c_{[2]} \otimes c_{[3]}$. 
Table 3: Arity values $n_{\star}$ of the polyadic convolution product (166), allowed by (167). The framed box corresponds to the binary convolution product.

\begin{tabular}{|c|c|c|c|c|c|c|c|c|c|c|c|c|c|}
\hline$n^{\prime} \quad n$ & $\mu$ & \multicolumn{3}{|c|}{$n=2$} & \multicolumn{3}{|c|}{$n=3$} & \multicolumn{3}{|c|}{$n=4$} & \multicolumn{3}{|c|}{$n=5$} \\
\hline$\Delta$ & $\ell_{\ell^{\prime}}^{\ell}$ & $\ell=1$ & $\ell=2$ & $\ell=3$ & $\ell=1$ & $\ell=2$ & $\ell=3$ & $\ell=1$ & $\ell=2$ & $\ell=3$ & $\ell=1$ & $\ell=2$ & $\ell=3$ \\
\hline \multirow{3}{*}{$n^{\prime}=2$} & $\ell^{\prime}=1$ & 2 & & & & & & & & & & & \\
\hline & $\ell^{\prime}=2$ & & 3 & & 3 & & & & & & & & \\
\hline & $\ell^{\prime}=3$ & & & 4 & & & & 4 & & & & & \\
\hline \multirow{3}{*}{$n^{\prime}=3$} & $\ell^{\prime}=1$ & & 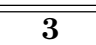 & & $\overline{73}$ & & & & & & & & \\
\hline & $\ell^{\prime}=2$ & & & & & 5 & & & & & 5 & & \\
\hline & $\ell^{\prime}=3$ & & & & & & 7 & & 7 & & & & \\
\hline \multirow{3}{*}{$n^{\prime}=4$} & $\ell^{\prime}=1$ & & & 4 & & & & 4 & & & & & \\
\hline & $\ell^{\prime}=2$ & & & & & & 7 & & 7 & & & & \\
\hline & $\ell^{\prime}=3$ & & & & & & & & & 10 & & & \\
\hline \multirow{3}{*}{$n^{\prime}=5$} & $\overline{\ell^{\prime}=1}$ & & & & & 5 & & & & & 5 & & \\
\hline & $\ell^{\prime}=2$ & & & & & & & & & & & 9 & \\
\hline & $\ell^{\prime}=3$ & & & & & & & & & & & & 13 \\
\hline
\end{tabular}

2. The algebra is binary $A^{(2)}=\langle A \mid \boldsymbol{\mu}\rangle$, and the coalgebra is ternary $C^{(3)}=\left\langle C \mid \boldsymbol{\Delta}^{(3)}\right\rangle$, which corresponds to $n=2, \ell=2, n^{\prime}=3, \ell^{\prime}=1$, the maps $\mathbf{f}^{(i)}: C^{\otimes 2} \rightarrow A, i=1,2,3$ in the elementwise description are two place, $\mathbf{f}^{(i)} \circ\left(c_{1} \otimes c_{2}\right)=f^{(i)}\left(c_{1}, c_{2}\right), c_{1,2} \in C$, and $\left(\Delta^{(3)}\right)^{\otimes 2}\left(c^{(1)} \otimes c^{(2)}\right)=\left(c_{[1]}^{(1)} \otimes c_{[2]}^{(1)} \otimes c_{[3]}^{(1)}\right) \otimes\left(c_{[1]}^{(2)} \otimes c_{[2]}^{(2)} \otimes c_{[3]}^{(2)}\right)$. The ternary convolution product is

$$
\boldsymbol{\mu}_{\star}^{(3)} \circ\left(\mathbf{f}^{(1)} \otimes \mathbf{f}^{(2)} \otimes \mathbf{f}^{(3)}\right) \circ\left(c^{(1)} \otimes c^{(2)}\right)=\mu^{\circ 2}\left[f^{(1)}\left(c_{[1]}^{(1)}, c_{[1]}^{(2)}\right), f^{(2)}\left(c_{[2]}^{(1)}, c_{[2]}^{(2)}\right), f^{(3)}\left(c_{[3]}^{(1)}, c_{[3]}^{(2)}\right)\right] .
$$

Example $94\left(\operatorname{Hom}_{\mathbb{k}}\left(C^{\otimes 2}, A^{\otimes 2}\right)\right)$. The last (fourth) possibility for the ternary convolution product (see TABLE $3)$ is nonderived and symmetric $n=3, \ell=1, n^{\prime}=3, \ell^{\prime}=1$, with both a ternary algebra $A^{(3)}=\left\langle A \mid \boldsymbol{\mu}^{(3)}\right\rangle$ and coalgebra $C^{(3)}=\left\langle C \mid \boldsymbol{\Delta}^{(3)}\right\rangle$. In the elementwise description the maps $\mathbf{f}^{(i)}: C^{\otimes 2} \rightarrow A^{\otimes 2}, i=1,2,3$ are $\mathbf{f}^{(i)} \circ\left(c_{1} \otimes c_{2}\right)=f_{[1]}^{(i)}\left(c_{1}, c_{2}\right) \otimes f_{[2]}^{(i)}\left(c_{1}, c_{2}\right), c_{1,2} \in C$. Then

$$
\begin{aligned}
\boldsymbol{\mu}_{\star}^{(3)} \circ\left(\mathbf{f}^{(1)} \otimes \mathbf{f}^{(2)} \otimes \mathbf{f}^{(3)}\right) \circ\left(c^{(1)} \otimes c^{(2)}\right) & =\mu^{(3)}\left[f_{[1]}^{(1)}\left(c_{[1]}^{(1)}, c_{[1]}^{(2)}\right), f_{[1]}^{(2)}\left(c_{[2]}^{(1)}, c_{[2]}^{(2)}\right), f_{[1]}^{(3)}\left(c_{[3]}^{(1)}, c_{[3]}^{(2)}\right)\right] \\
& \otimes \mu^{(3)}\left[f_{[2]}^{(1)}\left(c_{[1]}^{(1)}, c_{[1]}^{(2)}\right), f_{[2]}^{(2)}\left(c_{[2]}^{(1)}, c_{[2]}^{(2)}\right), f_{[2]}^{(3)}\left(c_{[3]}^{(1)}, c_{[3]}^{(2)}\right)\right] .
\end{aligned}
$$

The above examples present clearly the possible forms of the $n_{\star}$-ary convolution product, which can be convenient for lowest arity computations.

The general polyadic convolution product (166) in Sweedler notation can be presented as

$$
\begin{aligned}
& \boldsymbol{\mu}_{\star}^{\left(n_{\star}\right)} \circ\left(\mathbf{f}^{(1)} \otimes \mathbf{f}^{(2)} \otimes \ldots \otimes \mathbf{f}^{\left(n_{\star}\right)}\right)=\mathbf{g}, \quad \mathbf{f}^{(i)}, \mathbf{g} \in \operatorname{Hom}_{\mathbf{k}}\left(C^{\otimes\left(n^{\prime}-1\right)}, A^{\otimes(n-1)}\right), \\
& \mathbf{g}_{[j]} \circ\left(c^{(1)} \otimes \ldots \otimes c^{\left(n^{\prime}-1\right)}\right) \\
& =\left(\mu^{(n)}\right)^{\circ \ell}\left[f_{[j]}^{(1)}(\overbrace{c_{[1]}^{(1)}, \ldots, c_{[1]}^{\left(n^{\prime}-1\right)}}^{n^{\prime}-1}), f_{[j]}^{(2)}(\overbrace{c_{[2]}^{(1)}, \ldots, c_{[2]}^{\left(n^{\prime}-1\right)}}^{n^{\prime}-1}), \ldots, f_{[j]}^{\left(n_{\star}\right)}(\overbrace{c_{\left[n_{\star}\right]}^{(1)}, \ldots, c_{\left[n_{\star}\right]}^{\left(n^{\prime}-1\right)}}^{n^{\prime}-1})\right], \\
& f_{[j]}^{(i)} \in \operatorname{Hom}_{\mathbb{k}}\left(C^{\otimes\left(n^{\prime}-1\right)}, A\right), \quad i \in 1, \ldots, n_{\star}, \quad j \in 1, \ldots, n-1, \quad c \in C,
\end{aligned}
$$

where $\mathbf{g}_{[j]}$ are the Sweedler components of $\mathbf{g}$.

Recall that the associativity of the binary convolution product $(\star)$ is transparent in the Sweedler notation. Indeed, if $(f \star g) \circ(c)=f\left(c_{[1]}\right) \cdot g\left(c_{[2]}\right), f, g, h \in \operatorname{Hom}_{k}(C, A), c \in C,(\cdot) \equiv \mu_{A}^{(2)}$, then $((f \star g) \star h) \circ(c)=$ $\left(f\left(c_{[1]}\right) \cdot g\left(c_{[2]}\right)\right) \cdot h\left(c_{[3]}\right)=f\left(c_{[1]}\right) \cdot\left(g\left(c_{[2]}\right) \cdot h\left(c_{[3]}\right)\right)=(f \star(g \star h)) \circ(c)$. 
Lemma 95. The polyadic convolution algebra $C_{\star}^{\left(n^{\prime}, n\right)}$ (168) is associative.

Proof. To prove the claimed associativity of polyadic convolution $\boldsymbol{\mu}_{\star}^{\left(n_{\star}\right)}$ we express (5) in Sweedler notation. Starting from

$$
\mathbf{h}=\boldsymbol{\mu}_{\star}^{\left(n_{\star}\right)} \circ\left(\mathbf{g} \otimes \mathbf{f}^{\left(n_{\star}+1\right)} \otimes \mathbf{f}^{\left(n_{\star}+2\right)} \otimes \mathbf{.} \otimes \mathbf{f}^{\left(2 n_{\star}-1\right)}\right), \quad \mathbf{h} \in \operatorname{Hom}_{\mathbb{k}}\left(C^{\otimes\left(n^{\prime}-1\right)}, A^{\otimes(n-1)}\right),
$$

where $\mathbf{g}$ is given by (175), it follows that $\mathbf{h}$ should not depend of place of $\mathbf{g}$ in (176). Applying $\mathbf{h}$ to $c \in C$ twice, we obtain for its Sweedler components $\mathbf{h}_{[j]}, \quad j \in 1, \ldots, n-1$,

$$
\begin{aligned}
& \mathbf{h}_{[j]} \circ\left(c^{(1)} \otimes \ldots \otimes c^{\left(n^{\prime}-1\right)}\right)=\left(\mu^{(n)}\right)^{\circ \ell}\left[( \mu ^ { ( n ) } ) ^ { \propto \ell } \left[f_{[j]}^{(1)}(\overbrace{c_{[1]}^{(1)}, \ldots, c_{[1]}^{\left(n^{\prime}-1\right)}}^{n^{\prime}-1}), f_{[j]}^{(2)}(\overbrace{c_{[2]}^{(1)}, \ldots, c_{[2]}^{\left(n^{\prime}-1\right)}}^{n^{\prime}-1}),\right.\right. \\
& \left.\ldots, f_{[j]}^{\left(n_{\star}\right)}(\overbrace{c_{\left[2 n_{\star}-1\right]}^{(1)}, \ldots, c_{\left[2 n_{\star}-1\right]}^{\left(n^{\prime}-1\right)}}^{n^{\prime}-1})\right], f_{[j]}^{\left(n_{\star}+1\right)}(\overbrace{c_{[1]}^{(1)}, \ldots, c_{[1]}^{\left(n^{\prime}-1\right)}}^{n^{\prime}-1}), f_{[j]}^{\left(n_{\star}+2\right)}(\overbrace{c_{[2]}^{(1)}, \ldots, c_{[2]}^{\left(n^{\prime}-1\right)}}^{n^{\prime}-1}), \\
& \left.\ldots, f_{[j]}^{\left(2 n_{\star}-1\right)}(\overbrace{c_{\left[2 n_{\star}-1\right]}^{(1)}, \ldots, c_{\left[2 n_{\star}-1\right]}^{\left(n^{\prime}-1\right)}}^{n^{\prime}-1})\right]
\end{aligned}
$$

and here coassociativity and $(167)$ gives $\left(\Delta^{\left(n^{\prime}\right)}\right)^{\circ 2 l^{\prime}}(c)=c_{[1]} \otimes c_{[2]} \otimes \ldots \otimes c_{\left[2 n_{\star}-1\right]}$. Since $n$-ary algebra multiplication $\mu^{(n)}$ is associative, the internal $\left(\mu^{(n)}\right)^{\text {ol }}$ in (177) can be on any place, and $\mathbf{g}$ in (176) can be on any place as well. This means that the polyadic convolution product $\boldsymbol{\mu}_{\star}^{\left(n_{\star}\right)}$ is associative.

Observe the polyadic version of the identity used in (162): for any $\mathbf{f} \in \operatorname{Hom}_{\mathbb{k}}\left(C^{\otimes\left(n^{\prime}-1\right)}, A^{\otimes(n-1)}\right)$

$$
\mathrm{id}_{A}^{\otimes(n-1)} \circ \mathbf{f} \circ \mathrm{id}_{C}^{\otimes\left(n^{\prime}-1\right)}=\mathbf{f} .
$$

Proposition 96. If the polyadic associative algebra $A^{(n)}$ is unital with $\eta^{(r, n)}: K^{r} \rightarrow A^{\otimes(n-1)}$, and the polyadic coassociative coalgebra $C^{\left(n^{\prime}\right)}$ is counital with $\varepsilon^{\left(n^{\prime}, r^{\prime}\right)}: C^{\otimes\left(n^{\prime}-1\right)} \rightarrow K^{r^{\prime}}$, both over the same polyadic field $\mathbb{k}$, then the polyadic convolution algebra $C_{\star}^{\left(n^{\prime}, n\right)}$ (168) is unital, and its unit is given by $\boldsymbol{e}_{\star}^{\left(n^{\prime}, n\right)}(167)$.

Proof. In analogy with (162) we compose

$$
\begin{aligned}
& \mathbf{f}=\left(\left(\boldsymbol{\mu}^{(n)}\right)^{\circ \ell}\right)^{\otimes(n-1)} \circ \boldsymbol{\tau}_{\text {medial }}^{\left(n-1, n_{\star}\right)} \circ\left(\left(\boldsymbol{\eta}^{(r, n)}\right)^{\otimes\left(n_{\star}-1\right)} \otimes \mathrm{id}_{A}^{\otimes(n-1)}\right) \circ\left(\left(\boldsymbol{\gamma}^{\left(r^{\prime}, r\right)}\right)^{\otimes\left(n_{\star}-1\right)} \otimes \mathrm{id}_{A}^{\otimes(n-1)}\right) \\
& \circ\left(\left(\operatorname{id}_{K}^{\otimes r^{\prime}}\right)^{\otimes\left(n_{\star}-1\right)} \otimes \mathbf{f}\right) \circ\left(\left(\boldsymbol{\varepsilon}^{\left(n^{\prime}, r^{\prime}\right)}\right)^{\otimes\left(n_{\star}-1\right)} \otimes \mathrm{id}_{C}^{\otimes\left(n^{\prime}-1\right)}\right) \circ \boldsymbol{\tau}_{\text {medial }}^{\left(n_{\star}, n^{\prime}-1\right)} \circ\left(\left(\boldsymbol{\Delta}^{\left(n^{\prime}\right)}\right)^{\circ \ell^{\prime}}\right)^{\otimes\left(n^{\prime}-1\right)} \\
& =\left(\left(\boldsymbol{\mu}^{(n)}\right)^{\circ \ell}\right)^{\otimes(n-1)} \circ \boldsymbol{\tau}_{\text {medial }}^{\left(n-1, n_{\star}\right)} \circ\left(\boldsymbol{\eta}^{(r, n)} \circ \boldsymbol{\gamma}^{\left(r^{\prime}, r\right)} \circ \boldsymbol{\varepsilon}^{\left(n^{\prime}, r^{\prime}\right)}\right)^{\otimes\left(n_{\star}-1\right)} \\
& \circ\left(\mathrm{id}_{A}^{\otimes(n-1)} \circ \mathbf{f} \circ \mathrm{id}_{C}^{\otimes\left(n^{\prime}-1\right)}\right) \circ \boldsymbol{\tau}_{\text {medial }}^{\left(n_{\star}, n^{\prime}-1\right)} \circ\left(\left(\boldsymbol{\Delta}^{\left(n^{\prime}\right)}\right)^{\circ \ell^{\prime}}\right)^{\otimes\left(n^{\prime}-1\right)}=\boldsymbol{\mu}_{\star}^{\left(n_{\star}\right)} \circ\left(\left(\boldsymbol{e}_{\star}^{\left(n^{\prime}, n\right)}\right)^{\otimes\left(n_{\star}-1\right)} \otimes \mathbf{f}\right),
\end{aligned}
$$

which coincides with the polyadic unit definition (2). We use the identity (178) and the axioms for a polyadic unit (48) and counit (124). The same derivation can be made for any place of $\boldsymbol{\mu}_{\star}^{\left(n_{\star}\right)}$.

As in the general theory of $n$-ary groups [17], the invertibility of maps in $C_{\star}^{\left(n^{\prime}, n\right)}$ should be defined not by using the unit, but by using the querelement (4).

Definition 97. For a fixed $\mathbf{f} \in C_{\star}^{\left(n^{\prime}, n\right)}$ its coquerelement $\boldsymbol{q}_{\star}(\mathbf{f}) \in C_{\star}^{\left(n^{\prime}, n\right)}$ is the querelement in the $n_{\star}$-ary convolution product

$$
\boldsymbol{\mu}_{\star}^{\left(n_{\star}\right)} \circ\left(\mathbf{f}^{\otimes\left(n_{\star}-1\right)} \otimes \boldsymbol{q}_{\star}(\mathbf{f})\right)=\mathbf{f}
$$


where $\boldsymbol{q}_{\star}(\mathbf{f})$ can be on any place and $n_{\star} \geq 3$. The maps in a polyadic convolution algebra which have a coquerelement are called coquerable.

Define the positive convolution power $\ell_{\star}$ of an element $\mathbf{f} \in C_{\star}^{\left(n^{\prime}, n\right)}$ not recursively as in [36], but through the $\ell_{\star}$-iterated multiplication (6)

$$
\mathbf{f}^{\left\langle\ell_{\star}\right\rangle}=\left(\boldsymbol{\mu}_{\star}^{\left(n_{\star}\right)}\right)^{\circ \ell_{\star}} \circ\left(\mathbf{f}^{\otimes\left(\ell_{\star}\left(n_{\star}-1\right)+1\right)}\right),
$$

and an element in the negative convolution power $\mathbf{f}^{\left\langle-\ell_{\star}\right\rangle}$ satisfies the equation

$$
\left(\boldsymbol{\mu}_{\star}^{\left(n_{\star}\right)}\right)^{\circ \ell_{\star}} \circ\left(\mathbf{f}^{\left\langle\ell_{\star}-1\right\rangle} \otimes \mathbf{f}^{\otimes\left(n_{\star}-2\right)} \otimes \mathbf{f}^{\left\langle-\ell_{\star}\right\rangle}\right)=\left(\boldsymbol{\mu}_{\star}^{\left(n_{\star}\right)}\right)^{\circ \ell_{\star}} \circ\left(\mathbf{f}^{\otimes \ell_{\star}\left(n_{\star}-1\right)} \otimes \mathbf{f}^{\left\langle-\ell_{\star}\right\rangle}\right)=\mathbf{f} .
$$

It follows from (181) that the polyadic analogs of the exponent laws hold

$$
\begin{aligned}
& \boldsymbol{\mu}_{\star}^{\left(n_{\star}\right)} \circ\left(\mathbf{f}^{\left\langle\ell_{\star}^{(1)}\right\rangle} \otimes \mathbf{f}^{\left\langle\ell_{\star}^{(2)}\right\rangle} \otimes \ldots \otimes \mathbf{f}^{\left\langle\ell_{\star}^{\left(n_{\star}\right)}\right\rangle}\right)=\mathbf{f}^{\left\langle\ell_{\star}^{(1)}+\ell_{\star}^{(2)} \ldots+\ell_{\star}^{\left(n_{\star}\right)}+1\right\rangle}, \\
& \left(\mathbf{f}^{\left\langle\ell_{\star}^{(1)}\right\rangle}\right)^{\left\langle\ell_{\star}^{(2)}\right\rangle}=\mathbf{f}^{\left\langle\ell_{\star}^{(1)} \ell_{\star}^{(2)}\left(n_{\star}-1\right)+\ell_{\star}^{(1)}+\ell_{\star}^{(2)}\right\rangle} .
\end{aligned}
$$

Comparing (180) and (182), we have

$$
\boldsymbol{q}_{\star}(\mathbf{f})=\mathbf{f}^{\langle-1\rangle} .
$$

An arbitrary polyadic power $\ell_{Q}$ of the coquerelement $\boldsymbol{q}_{\star}^{\circ \ell_{Q}}(\mathbf{f})$ is defined by (180) recursively and can be expressed through the negative polyadic power of $\mathbf{f}$ (see, e.g. [37] for $n$-ary groups). In terms of the Heine numbers [38] (or $q$-deformed numbers [39])

$$
[[l]]_{q}=\frac{q^{l}-1}{q-1}, \quad l \in \mathbb{N}_{0}, \quad q \in \mathbb{Z}
$$

we obtain [14]

$$
\boldsymbol{q}_{\star}^{\circ \ell_{Q}}(\mathbf{f})=\mathbf{f}^{\left\langle-\left[\left[\ell_{Q}\right]\right]_{2-n_{\star}}\right\rangle} .
$$

\section{POLYADIC BIALGEBRAS}

The next step is to combine algebras and coalgebras into a common algebraic structure in some "natural" way. Informally, a bialgebra is defined as a vector space which is "simultaneously" an algebra and a coalgebra with some compatibility conditions (e.g., $[1,2])$.

In search of a polyadic analog of bialgebras, we observe two structural differences with the binary case: 1) since the unit and counit do not necessarily exist, we obtain 4 different kinds of bialgebras (similar to the unit and zero in TABLE 1); 2 ) where the most exotic is the possibility of unequal arities of multiplication and comultiplication $n \neq n^{\prime}$. Initially, we take them as arbitrary and then try to find restrictions arising from some "natural" relations.

Let $B_{v e c t}$ be a polyadic vector space over the polyadic field $\mathbb{k}^{\left(m_{k}, n_{k}\right)}$ as (see (11) and (109))

$$
B_{\text {vect }}=\left\langle B, K \mid \nu^{(m)} ; \nu_{k}^{\left(m_{k}\right)}, \mu_{k}^{\left(n_{k}\right)} ; \rho^{(r)}\right\rangle
$$

where $\nu^{(m)}: B^{\times m} \rightarrow B$ is $m$-ary addition and $\rho^{(r)}: K^{\times r} \times B \rightarrow B$ is $r$-place action (see (7)).

Definition 98. A polyadic bialgebra $B^{\left(n^{\prime}, n\right)}$ is $B_{\text {vect }}$ equipped with a $\mathbb{k}$-linear $n$-ary multiplication map $\boldsymbol{\mu}^{(n)}$ : $B^{\otimes n} \rightarrow B$ and a $\mathbb{k}$-linear $n^{\prime}$-ary comultiplication map $\boldsymbol{\Delta}^{\left(n^{\prime}\right)}: B \rightarrow B^{\otimes n^{\prime}}$ such that:

1. (a) $B_{A}^{(n)}=\left\langle B_{v e c t} \mid \boldsymbol{\mu}^{(n)}\right\rangle$ is a $n$-ary algebra;

(б) The map $\boldsymbol{\mu}^{(n)}$ is a coalgebra (homo)morphism (129).

2. (a) $B_{C}^{\left(n^{\prime}\right)}=\left\langle B_{\text {vect }} \mid \boldsymbol{\Delta}^{\left(n^{\prime}\right)}\right\rangle$ is a $n^{\prime}$-ary coalgebra;

(б) The map $\boldsymbol{\Delta}^{\left(n^{\prime}\right)}$ is an algebra (homo)morphism (86). 
The equivalence of the compatibility conditions $\mathbf{1} b$ ) and $\mathbf{2} b$ ) can be expressed in the form (polyadic analog of " $\Delta \circ \mu=(\mu \otimes \mu)($ id $\otimes \tau \otimes$ id $)(\Delta \otimes \Delta)$ ")

$$
\boldsymbol{\Delta}^{\left(n^{\prime}\right)} \circ \boldsymbol{\mu}^{(n)}=(\overbrace{\boldsymbol{\mu}^{(n)} \otimes \ldots \otimes \boldsymbol{\mu}^{(n)}}^{n^{\prime}}) \circ \boldsymbol{\tau}_{\text {medial }}^{\left(n, n^{\prime}\right)} \circ(\overbrace{\boldsymbol{\Delta}^{\left(n^{\prime}\right)} \otimes \ldots \otimes \boldsymbol{\Delta}^{\left(n^{\prime}\right)}}^{n}),
$$

where $\boldsymbol{\tau}_{\text {medial }}^{\left(n, n^{\prime}\right)}$ is the medial map (69) acting on $B$, while the diagram

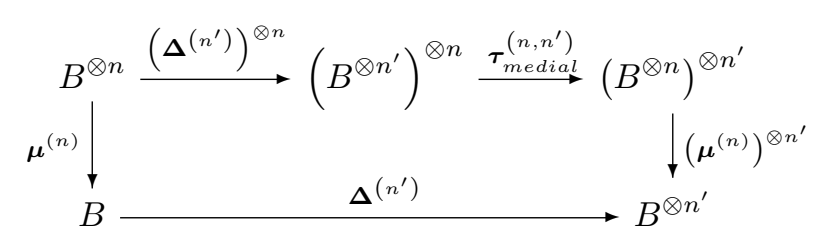

commutes.

In an elementwise description it is the commutation of $n$-ary multiplication and $n^{\prime}$-ary comultiplication

$$
\Delta^{\left(n^{\prime}\right)}\left(\mu^{(n)}\left[b_{1}, \ldots, b_{n}\right]\right)=\mu^{(n)}\left[\Delta^{\left(n^{\prime}\right)}\left(b_{1}\right), \ldots, \Delta^{\left(n^{\prime}\right)}\left(b_{n}\right)\right],
$$

which in the Sweedler notation becomes

$$
\begin{aligned}
& \mu^{(n)}\left[b_{1}, \ldots, b_{n}\right]_{[1]} \otimes \mu^{(n)}\left[b_{1}, \ldots, b_{n}\right]_{[2]} \otimes \ldots \otimes \mu^{(n)}\left[b_{1}, \ldots, b_{n}\right]_{\left[n^{\prime}\right]} \\
& =\mu^{(n)}\left[b_{[1]}^{(1)}, \ldots, b_{[1]}^{(n)}\right] \otimes \mu^{(n)}\left[b_{[2]}^{(1)}, \ldots, b_{[2]}^{(n)}\right] \otimes \ldots \otimes \mu^{(n)}\left[b_{\left[n^{\prime}\right]}^{(1)}, \ldots, b_{\left[n^{\prime}\right]}^{(n)}\right] .
\end{aligned}
$$

Consider the example of a nonderived bialgebra $B^{(n, n)}$ which follows from the von Neumann higher $n$ regularity relations [40-43].

Example 99 (von Neumann n-regular bialgebra). Let $B^{(n, n)}=\left\langle B \mid \boldsymbol{\mu}^{(n)}, \boldsymbol{\Delta}^{(n)}\right\rangle$ be a polyadic bialgebra generated by the elements $b_{i} \in B, \quad i=1, \ldots, n-1$ subject to the nonderived $n$-ary multiplication

$$
\begin{aligned}
\mu^{(n)}\left(b_{1}, b_{2}, b_{3} \ldots, b_{n-2}, b_{n-1}, b_{1}\right) & =b_{1}, \\
\mu^{(n)}\left(b_{2}, b_{3}, b_{4} \ldots b_{n-1}, b_{1}, b_{2}\right) & =b_{2}, \\
& \vdots \\
\mu^{(n)}\left(b_{n-1}, b_{1}, b_{2} \ldots b_{n-3}, b_{n-2}, b_{n-1}\right) & =b_{n-1},
\end{aligned}
$$

and the nonderived $n$-ary comultiplication (cf. 119)

$$
\begin{aligned}
\Delta^{(n)}\left(b_{1}\right) & =b_{1} \otimes b_{2} \otimes b_{3} \ldots, b_{n-2} \otimes b_{n-1} \otimes b_{1}, \\
\Delta^{(n)}\left(b_{2}\right) & =b_{2} \otimes b_{3} \otimes b_{4} \ldots, b_{n-1} \otimes b_{1} \otimes b_{2}, \\
\vdots & \\
\Delta^{(n)}\left(b_{n-1}\right) & =b_{n-1} \otimes b_{1} \otimes b_{2} \otimes \ldots \otimes b_{n-3} \otimes b_{n-2} \otimes b_{n-1} .
\end{aligned}
$$

It is straightforward to check that the compatibility condition (191) holds. Many possibilities exist for choosing other operations - algebra addition, field addition and multiplication, action - so to demonstrate the compatibility we have confined ourselves to only the algebra multiplication and comultiplication.

If the $n$-ary algebra $B_{A}^{(n)}$ has unit and/or $n^{\prime}$-ary coalgebra $B_{C}^{\left(n^{\prime}\right)}$ has counit $\varepsilon^{\left(n^{\prime}, r^{\prime}\right)}$, we should add the following additional axioms.

Definition 100 (Unit axiom). If $B_{A}^{(n)}=\left\langle B_{v e c t} \mid \boldsymbol{\mu}^{(n)}\right\rangle$ is unital, then the unit $\boldsymbol{\eta}^{(r, n)}$ is a (homo)morphism of the coalgebra $B_{C}^{\left(n^{\prime}\right)}=\left\langle B_{\text {vect }} \mid \boldsymbol{\Delta}^{\left(n^{\prime}\right)}\right\rangle($ see (129))

$$
(\overbrace{\boldsymbol{\Delta}^{\left(n^{\prime}\right)} \otimes \ldots \otimes \boldsymbol{\Delta}^{\left(n^{\prime}\right)}}^{n-1}) \circ \boldsymbol{\eta}^{(r, n)}=(\overbrace{\boldsymbol{\eta}^{(r, n)} \otimes \ldots \otimes \boldsymbol{\eta}^{(r, n)}}^{n^{\prime}})
$$


such that the diagram

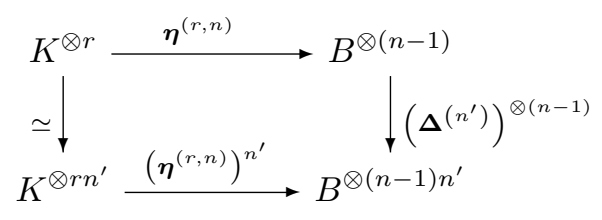

commutes.

Definition 101 (Counit axiom). If $B_{C}^{\left(n^{\prime}\right)}=\left\langle B_{v e c t} \mid \boldsymbol{\Delta}^{\left(n^{\prime}\right)}\right\rangle$ is counital, then the counit $\varepsilon^{\left(n^{\prime}, r^{\prime}\right)}$ is a (homo)morphism of the algebra $B_{A}^{(n)}=\left\langle B_{\text {vect }} \mid \boldsymbol{\mu}^{(n)}\right\rangle$ (see (86))

$$
\varepsilon^{\left(n^{\prime}, r^{\prime}\right)} \circ(\overbrace{\boldsymbol{\mu}^{(n)} \otimes \ldots \otimes \boldsymbol{\mu}^{(n)}}^{n^{\prime}-1})=(\overbrace{\varepsilon^{\left(n^{\prime}, r^{\prime}\right)} \otimes \ldots \otimes \varepsilon^{\left(n^{\prime}, r^{\prime}\right)}}^{n})
$$

such that the diagram

commutes.

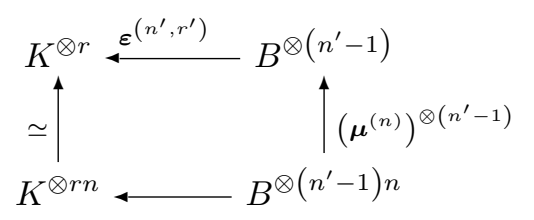

If both the polyadic unit and polyadic counit exist, then we include their compatibility condition

$$
\left(\varepsilon^{\left(n^{\prime}, r^{\prime}\right)}\right)^{\otimes(n-1)} \circ\left(\boldsymbol{\eta}^{(r, n)}\right)^{\otimes\left(n^{\prime}-1\right)} \simeq \mathrm{id}_{K},
$$

such that the diagram

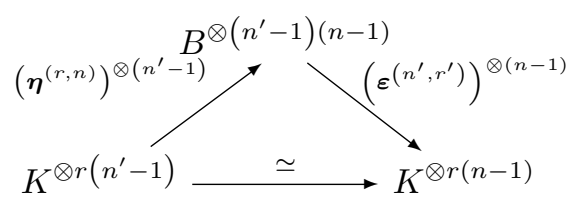

commutes.

Assertion 102. There are four kinds of polyadic bialgebras depending on whether the unit $\boldsymbol{\eta}^{(r, n)}$ and counit $\varepsilon^{\left(n^{\prime}, r^{\prime}\right)}$ exist:

1) nonunital-noncounital; 2) unital-noncounital; 3) nonunital-counital; 4) unital-counital.

Definition 103. A polyadic bialgebra $B^{\left(n^{\prime}, n\right)}$ is called totally co-commutative, if

$$
\begin{gathered}
\boldsymbol{\mu}^{(n)}=\boldsymbol{\mu}^{(n)} \circ \boldsymbol{\tau}_{n}, \\
\boldsymbol{\Delta}^{\left(n^{\prime}\right)}=\boldsymbol{\tau}_{n^{\prime}} \circ \boldsymbol{\Delta}^{\left(n^{\prime}\right),}
\end{gathered}
$$

where $\boldsymbol{\tau}_{n} \in S_{n}, \boldsymbol{\tau}_{n^{\prime}} \in S_{n^{\prime}}$, and $S_{n}, S_{n^{\prime}}$ are the symmetry permutation groups on $n$ and $n^{\prime}$ elements respectively.

Definition 104. A polyadic bialgebra $B^{\left(n^{\prime}, n\right)}$ is called medially co-commutative, if

$$
\begin{gathered}
\boldsymbol{\mu}_{o p}^{(n)} \equiv \boldsymbol{\mu}^{(n)} \circ \boldsymbol{\tau}_{o p}^{(n)}=\boldsymbol{\mu}^{(n)}, \\
\boldsymbol{\Delta}_{c o p}^{\left(n^{\prime}\right)} \equiv \boldsymbol{\tau}_{o p}^{\left(n^{\prime}\right)} \circ \boldsymbol{\Delta}^{\left(n^{\prime}\right)}=\boldsymbol{\Delta}^{\left(n^{\prime}\right)},
\end{gathered}
$$

where $\boldsymbol{\tau}_{o p}^{(n)}$ and $\boldsymbol{\tau}_{o p}^{\left(n^{\prime}\right)}$ are the medially allowed polyadic twist maps (72). 


\section{POLYADIC HOPF ALGEBRAS}

Here we introduce the most general approach to "polyadization" of the Hopf algebra concept [1-3]. Informally, the transition from bialgebra to Hopf algebra is, in some sense, "dualizing" the passage from semigroup (containing noninvertible elements) to group (in which all elements are invertible). Schematically, if multiplication $\mu=(\cdot)$ in a semigroup $G$ is binary, the invertibility of all elements demands two extra and necessary set-ups: 1) An additional element (identity $e \in G$ or the corresponding map from a one point set to group $\epsilon$ ); 2 ) An additional map (inverse $\iota: G \rightarrow G$ ), such that $g \cdot \iota(g)=e$ in diagrammatic form is $\mu \circ\left(\operatorname{id}_{G} \times \iota\right) \circ D_{2}=\epsilon$ ( $D_{2}: G \rightarrow G \times G$ is the diagonal map). When "dualizing", in a (binary) bialgebra $B$ (with multiplication $\mu$ and comultiplication $\Delta$ ) again two set-ups should be considered in order to get a (binary) Hopf algebra: 1) An analog of identity $e_{\star}=\eta \varepsilon$ (where $\eta: k \rightarrow B$ is unit and $\varepsilon: B \rightarrow k$ is counit); 2) An analog of inverse $S: B \rightarrow B$ called the antipode, such that $\mu \circ\left(\operatorname{id}_{B} \otimes S\right) \circ \Delta=e_{\star}$ or in terms of the (binary) convolution product $\operatorname{id}_{B} \star S=e_{\star}$. By multiplying both sides by $S$ from the left and by $\operatorname{id}_{B}$ from the right, we obtain weaker (von Neumann regularity) conditions $S \star \operatorname{id}_{B} \star S=S, \operatorname{id}_{B} \star S \star \operatorname{id}_{B}=\operatorname{id}_{B}$, which do not contain an identity $e_{\star}$ and lead to the concept of weak Hopf algebras [44-46].

The crucial peculiarity of the polyadic generalization is the possible absence of an identity or 1 ) in both cases. The role and necessity of the polyadic identity (2) is not so important: there polyadic groups without identity exist (see, e.g. [47], and the discussion after (3)). Invertibility is determined by the querelement (4) in $n$-ary group or the quermap (57) in polyadic algebra. So there are two ways forward: "dualize" the quermap (57) directly (as in the binary case) or use the most general version of the polyadic convolution product (166) and apply possible restrictions, if any. We will choose the second method, because the first one is a particular case of it. Thus, if the standard (binary) antipode is the convolution inverse (coinverse) to the identity in a bialgebra, then its polyadic counterpart should be a coquerelement (180) of some polyadic analog for the identity map in the polyadic bialgebra. We consider two possibilities to define a polyadic analog of identity: 1) Singular case. The comultiplication is binary $\left.n^{\prime}=2 ; \mathbf{2}\right)$ Symmetric case. The arities of multiplication and comultiplication need not be binary, but should coincide $n=n^{\prime}$.

In the singular case a polyadic multivalued map in $\operatorname{End}_{\mathbb{k}}\left(B, B^{\otimes(n-1)}\right)$ is a reminder of how an identity can be defined: its components are to be functions of one variable. That is, with more than one argument it is not possible to determine its value when these are unequal.

Definition 105. We take for a singular polyadic identity $\mathbf{I d}_{0}$ the diagonal map $\mathbf{I d}_{0}=\mathbf{D} \in \operatorname{End}_{\mathbb{k}}\left(B, B^{\otimes(n-1)}\right)$, such that $b \mapsto b^{\otimes(n-1)}$, for any $b \in B$.

We call the polyadic convolution product (166) with the binary comultiplication $n^{\prime}=2$ reduced and denote it by $\overline{\boldsymbol{\mu}}_{\star}^{\left(n_{\star}\right)}$ which in Sweedler notation can be obtained from (175)

$$
\begin{aligned}
& \overline{\boldsymbol{\mu}}_{\star}^{\left(n_{\star}\right)} \circ\left(\mathbf{f}^{(1)} \otimes \mathbf{f}^{(2)} \otimes \ldots \otimes \mathbf{f}^{\left(n_{\star}\right)}\right)=\mathbf{g}, \quad \mathbf{f}^{(i)}, \mathbf{g} \in \operatorname{End}_{\mathbb{k}}\left(B, B^{\otimes(n-1)}\right), \\
& \mathbf{g}_{[j]} \circ(b)=\left(\mu^{(n)}\right)^{\circ \ell}\left[f_{[j]}^{(1)}\left(b_{[1]}\right), f_{[j]}^{(2)}\left(b_{[2]}\right), \ldots, f_{[j]}^{\left(n_{\star}\right)}\left(b_{\left[n_{\star}\right]}\right)\right], \\
& f_{[j]}^{(i)} \in \operatorname{End}_{\mathbb{k}}(B, B), \quad i \in 1, \ldots, n_{\star}, \quad j \in 1, \ldots, n-1, \quad b \in B,
\end{aligned}
$$

The consistency condition (167) becomes reduced

$$
n_{\star}=\ell(n-1)+1=\ell^{\prime}+1 .
$$

Definition 106. The set of the multivalued maps $\mathbf{f}^{(i)} \in \operatorname{End}_{\mathrm{k}}\left(B, B^{\otimes(n-1)}\right)$ (together with the polyadic identity $\mathbf{I d}_{0}$ ) endowed with the reduced convolution product $\overline{\boldsymbol{\mu}}_{\star}^{\left(n_{\star}\right)}$ is called a reduced $n_{\star}$-ary convolution algebra

$$
C_{\star}^{(2, n)}=\left\langle\operatorname{End}_{\mathbb{k}}\left(B, B^{\otimes(n-1)}\right) \mid \overline{\boldsymbol{\mu}}_{\star}^{\left(n_{\star}\right)}\right\rangle .
$$

Remark 107. The reduced convolution algebra $C_{\star}^{(2, n)}$ having $n \neq 2$ is not derived (Definition 89).

Having the distinguished element $\mathbf{I d}_{0} \in C_{\star}^{(2, n)}$ as an analog of $\operatorname{id}_{B}$ and the querelement (180) for any $\mathbf{f} \in C_{\star}^{(2, n)}$ (the polyadic version of inverse in the convolution algebra), we are now in a position to "polyadize" the concept of the (binary) antipode.

Definition 108. A multivalued map $\boldsymbol{Q}_{0}: B \rightarrow B^{\otimes(n-1)}$ in the polyadic bialgebra $B^{(2, n)}$ is called a singular querantipode, if it is the coquerelement of the polyadic identity $\boldsymbol{Q}_{0}=\boldsymbol{q}_{\star}\left(\mathbf{I d}_{0}\right)$ in the reduced $n_{\star}$-ary convolution algebra

$$
\overline{\boldsymbol{\mu}}_{\star}^{\left(n_{\star}\right)} \circ\left(\mathbf{I d}_{0}^{\otimes\left(n_{\star}-1\right)} \otimes \boldsymbol{Q}_{0}\right)=\mathbf{I} \mathbf{d}_{0},
$$


where $\boldsymbol{Q}_{0}$ can be on any place, such that the diagram

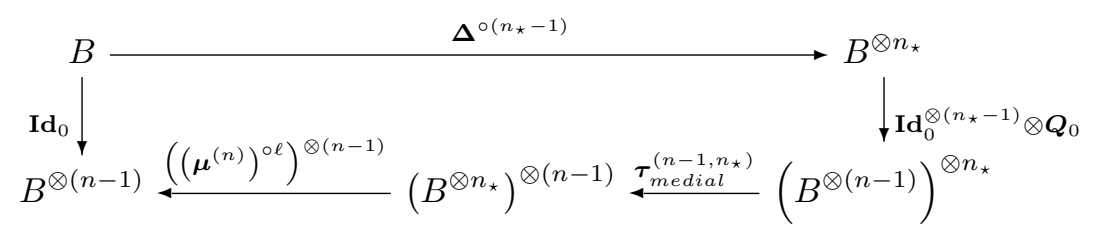

commutes.

In Sweedler notation

$$
\left(\mu^{(n)}\right)^{\circ \ell}\left[b_{[1]}, b_{[2]}, \ldots, b_{\left[n_{\star}-1\right]}, Q_{0[j]}\left(b_{\left[n_{\star}\right]}\right)\right]=b, \quad j \in 1, \ldots, n-1, \quad i \in 1, \ldots, n_{\star},
$$

where

$$
\boldsymbol{\Delta}^{\circ\left(n_{\star}-1\right)}(b)=b_{[1]} \otimes b_{[2]} \otimes \ldots \otimes b_{\left[n_{\star}\right]}, \boldsymbol{Q}_{0} \circ(b)=Q_{0[1]}(b) \otimes \ldots \otimes Q_{0[n-1]}(b), b, b_{[i]} \in B
$$

Definition 109. A polyadic bialgebra $B^{(2, n)}$ equipped with the reduced $n_{\star}$-ary convolution product $\overline{\boldsymbol{\mu}}_{\star}^{\left(n_{\star}\right)}$ and the singular querantipode $\boldsymbol{Q}_{0}(212)$ is called a singular polyadic Hopf algebra and is denoted by $H_{\text {sing }}^{(n)}=$ $\left\langle B^{(n, n)} \mid \overline{\boldsymbol{\mu}}_{\star}^{\left(n_{\star}\right)}, \boldsymbol{Q}_{0}\right\rangle$.

Due to their exotic properties we will not consider singular polyadic Hopf algebras $H_{\text {sing }}^{(n)}$ in detail.

In the symmetric case a polyadic identity-like map in $\operatorname{End}_{\mathbb{k}}\left(B^{\otimes(n-1)}, B^{\otimes(n-1)}\right)$ can be defined in a more natural way.

Definition 110. A symmetric polyadic identity Id : $B^{\otimes(n-1)} \rightarrow B^{\otimes(n-1)}$ is a polyadic tensor product of ordinary identities in $B^{(n, n)}$

$$
\mathbf{I d}=\overbrace{\mathrm{id}_{B} \otimes \ldots \otimes \operatorname{id}_{B}}^{n-1}, \quad \operatorname{id}_{B}: B \rightarrow B .
$$

Indeed, for any map $\mathbf{f} \in \operatorname{End}_{\mathbb{k}}\left(B^{\otimes(n-1)}, B^{\otimes(n-1)}\right)$, obviously $\mathbf{I d} \circ \mathbf{f}=\mathbf{f} \circ \mathbf{I d}=\mathbf{f}$.

The numbers of iterations are now equal $\ell=\ell^{\prime}$, and the consistency condition (167) becomes

$$
n_{\star}-1=\ell(n-1) \text {. }
$$

Definition 111. The set of the multiplace multivalued maps $\mathbf{f}^{(i)} \in \operatorname{End}_{\mathbb{k}}\left(B^{\otimes(n-1)}, B^{\otimes(n-1)}\right)$ (together with the polyadic identity Id) endowed with the symmetric convolution product $\hat{\boldsymbol{\mu}}_{\star}^{\left(n_{\star}\right)}=\left.\boldsymbol{\mu}_{\star}^{\left(n_{\star}\right)}\right|_{n=n^{\prime}}(166)$ is called a symmetric $n_{\star}$-ary convolution algebra

$$
C_{\star}^{(n, n)}=\left\langle\operatorname{End}_{\mathbb{k}}\left(B^{\otimes(n-1)}, B^{\otimes(n-1)}\right) \mid \hat{\boldsymbol{\mu}}_{\star}^{\left(n_{\star}\right)}\right\rangle .
$$

For a polyadic analog of antipode in the symmetric case we have

Definition 112. A multiplace multivalued map $\boldsymbol{Q}_{\text {id }}: B^{\otimes(n-1)} \rightarrow B^{\otimes(n-1)}$ in the polyadic bialgebra $B^{(n, n)}$ is called a symmetric querantipode, if it is the coquerelement (see (180)) of the polyadic identity $\boldsymbol{Q}_{\text {id }}=\boldsymbol{q}_{\star}(\mathbf{I d})$ in the symmetric $n_{\star}$-ary convolution algebra

$$
\hat{\boldsymbol{\mu}}_{\star}^{\left(n_{\star}\right)} \circ\left(\mathbf{I d}^{\otimes\left(n_{\star}-1\right)} \otimes \boldsymbol{Q}_{\text {id }}\right)=\mathbf{I d},
$$

where $\boldsymbol{Q}_{\text {id }}$ can be on any place, such that the diagram

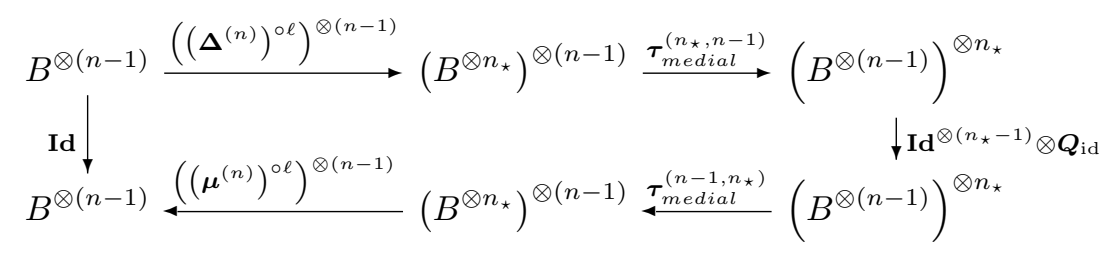

commutes. 
In Sweedler notation we obtain (see (166) and (175))

$$
\left(\mu^{(n)}\right)^{\propto \ell}\left[b_{[1]}^{(j)}, b_{[2]}^{(j)}, b_{\left[n_{\star}-1\right]}^{(j)}, Q_{[j]}\left(b_{\left[n_{\star}\right]}^{(1)}, b_{\left[n_{\star}\right]}^{(2)}, \ldots, b_{\left[n_{\star}\right]}^{(n-1)}\right)\right]=b^{(j)}, j \in 1, \ldots, n-1, i \in 1, \ldots, n_{\star},
$$

where $\left(\Delta^{(n)}\right)^{\propto \ell}\left(b^{(j)}\right)=b_{[1]}^{(j)} \otimes b_{[2]}^{(j)} \otimes \ldots \otimes b_{\left[n_{\star}\right]}^{(j)}, \quad b_{[i]}^{(j)} \in B, \ell \in \mathbb{N}, Q_{[j]} \in \operatorname{End}_{\mathbb{k}}\left(B^{\otimes(n-1)}, B\right)$ are components of $\boldsymbol{Q}_{\mathrm{id}}$, and the convolution product arity is $n_{\star}=\ell(n-1)+1(216)$.

Definition 113. A polyadic bialgebra $B^{(n, n)}$ equipped with the symmetric $n_{\star}$-ary convolution product $\hat{\boldsymbol{\mu}}_{\star}^{\left(n_{\star}\right)}$ and the symmetric querantipode $\boldsymbol{Q}_{\mathrm{id}}(218)$ is called a symmetric polyadic Hopf algebra and is denoted by $H_{s y m}^{(n)}=\left\langle B^{(n, n)} \mid \hat{\boldsymbol{\mu}}_{\star}^{\left(n_{\star}\right)}, \boldsymbol{Q}_{\mathrm{id}}\right\rangle$.

Example 114. In the case where $n=n^{\prime}=3$ and $\ell=1$ we have $\Delta^{(3)}\left(b^{(j)}\right)=b_{[1]}^{(j)} \otimes b_{[2]}^{(j)} \otimes b_{[3]}^{(j)}, j=1,2,3$,

$$
\begin{array}{ll}
\mu^{(3)}\left[b_{[1]}^{(1)}, b_{[2]}^{(1)}, Q_{[1]}\left(b_{[3]}^{(1)}, b_{[3]}^{(2)}\right)\right]=b^{(1)}, & \mu^{(3)}\left[b_{[1]}^{(2)}, b_{[2]}^{(2)}, Q_{[2]}\left(b_{[3]}^{(1)}, b_{[3]}^{(2)}\right)\right]=b^{(2)}, \\
\mu^{(3)}\left[b_{[1]}^{(1)}, Q_{[1]}\left(b_{[2]}^{(1)}, b_{[2]}^{(2)}\right), b_{[3]}^{(1)}\right]=b^{(1)}, & \mu^{(3)}\left[b_{[1]}^{(2)}, Q_{[2]}\left(b_{[2]}^{(1)}, b_{[2]}^{(2)}\right), b_{[3]}^{(2)}\right]=b^{(2)}, \\
\mu^{(3)}\left[Q_{[1]}\left(b_{[1]}^{(1)}, b_{[1]}^{(2)}\right), b_{[2]}^{(1)}, b_{[3]}^{(1)}\right]=b^{(1)}, & \mu^{(3)}\left[Q_{[2]}\left(b_{[1]}^{(1)}, b_{[1]}^{(2)}\right), b_{[2]}^{(2)}, b_{[3]}^{(2)}\right]=b^{(2)},
\end{array}
$$

which can be compared with the binary case $\left(b_{[1]} S\left(b_{[2]}\right)=S\left(b_{[1]}\right) b_{[2]}=\eta(\varepsilon(b))\right)$ and (4), (180).

Recall that the main property of the antipode $S$ of a binary bialgebra $B$ is its "anticommutation" with the multiplication $\mu$ and comultiplication $\Delta$ (e.g., [1])

$$
\begin{array}{rlrl}
S \circ \mu & =\mu \circ \tau_{o p} \circ(S \otimes S), & & S \circ \eta=\eta, \\
\Delta \circ S=\tau_{o p} \circ(S \otimes S) \circ \Delta, & \varepsilon \circ S=\varepsilon,
\end{array}
$$

where $\tau_{o p}$ is the binary twist (see (66)). The first relation means that $S$ is an algebra anti-endomorphism, because in the elementwise description $S(a \cdot b)=S(b) \cdot S(a), a, b \in B,(\cdot) \equiv \mu$.

We propose the polyadic analogs of (222)-(223) without proofs, which are too cumbersome, but their derivations almost coincide with those for the binary case.

Proposition 115. The querantipode $\boldsymbol{Q}_{\mathrm{id}}: B^{\otimes(n-1)} \rightarrow B^{\otimes(n-1)}$ of the polyadic bialgebra $B^{(n, n)}=\left\langle B \mid \boldsymbol{\mu}^{(n)}, \boldsymbol{\Delta}^{(n)}\right\rangle$ satisfies the polyadic version of "antimultiplicativity" ("antialgebra map")

$$
\boldsymbol{Q}_{\mathrm{id}} \circ\left(\left(\boldsymbol{\mu}^{(n)}\right)^{\circ \ell}\right)^{\otimes(n-1)}=\left(\left(\boldsymbol{\mu}^{(n)}\right)^{\circ \ell}\right)^{\otimes(n-1)} \circ \boldsymbol{\tau}_{o p}^{\left(\ell_{\tau}\right)} \circ \boldsymbol{Q}_{\mathrm{id}}^{\otimes n_{\star}} \circ \boldsymbol{\tau}_{\text {medial }}^{\left(n_{\star}, n-1\right)},
$$

and "anticomultiplicativity" ("anticoalgebra map")

$$
\left(\left(\boldsymbol{\Delta}^{(n)}\right)^{\circ \ell}\right)^{\otimes(n-1)} \circ \boldsymbol{Q}_{\mathrm{id}}=\boldsymbol{\tau}_{o p}^{\left(\ell_{\tau}\right)} \circ \boldsymbol{Q}_{\mathrm{id}}^{\otimes n_{\star}} \circ \boldsymbol{\tau}_{\text {medial }}^{\left(n-1, n_{\star}\right)} \circ\left(\left(\boldsymbol{\Delta}^{(n)}\right)^{\circ \ell}\right)^{\otimes(n-1)},
$$

where $\boldsymbol{\tau}_{\text {medial }}^{(n, m)}$ is the medial map $(69), \boldsymbol{\tau}_{o p}^{\left(\ell_{\tau}\right)}$ is the polyadic twist $(72)$ and $\ell_{\tau}=(n-1) n_{\star}$ should be allowed (see TABLE 2).

Proposition 116. If the polyadic unit $\boldsymbol{\eta}^{(r, n)}(48)$ and counit $\boldsymbol{\varepsilon}^{(n, r)}(124)$ in $B^{(n, n)}$ exist, then

$$
\begin{aligned}
& \boldsymbol{Q}_{\mathrm{id}} \circ \boldsymbol{\eta}^{(r, n)}=\boldsymbol{\eta}^{(r, n)}, \\
& \boldsymbol{\varepsilon}^{(n, r)} \circ \boldsymbol{Q}_{\mathrm{id}}=\boldsymbol{\varepsilon}^{(n, r)} .
\end{aligned}
$$

Example 117. If $n=3, \ell=1, n_{\star}=3, \ell_{\tau}=6$ (and (75)), then using Sweedler notation, for (224) we have

$$
\begin{aligned}
& Q_{[1]}\left(\mu^{(3)}\left[a_{1}, a_{2}, a_{3}\right], \mu^{(3)}\left[b_{1}, b_{2}, b_{3}\right]\right)=\mu^{(3)}\left[Q_{[2]}\left(a_{2}, b_{2}\right), Q_{[1]}\left(a_{1}, b_{1}\right), Q_{[1]}\left(a_{3}, b_{3}\right)\right], \\
& Q_{[2]}\left(\mu^{(3)}\left[a_{1}, a_{2}, a_{3}\right], \mu^{(3)}\left[b_{1}, b_{2}, b_{3}\right]\right)=\mu^{(3)}\left[Q_{[2]}\left(a_{1}, b_{1}\right), Q_{[2]}\left(a_{3}, b_{3}\right), Q_{[1]}\left(a_{2}, b_{2}\right)\right],
\end{aligned}
$$

where $\boldsymbol{Q}_{\mathrm{id}} \circ(a, b)=Q_{[1]}(a, b) \otimes Q_{[2]}(a, b) \in \operatorname{End}_{\mathbb{k}}(B \otimes B, B \otimes B), a, b, a_{i}, b_{i} \in B$, (cf. $\left.(222)\right)$. 
The key property of the binary antipode $S$ is its involutivity $S^{\circ 2}=\operatorname{id}_{B}$ for either commutative $\left(\mu=\mu \circ \tau_{o p}\right)$ or co-commutative $\left(\Delta=\tau_{o p} \circ \Delta\right)$ Hopf algebras, which follows from (222) or (223) applied to $S \star S^{\circ 2}$ giving $\eta \varepsilon$ $\left(=S \star \operatorname{id}_{B}\right)$.

Proposition 118. If in a symmetric Hopf algebra $H_{\text {sym }}^{(n)}$ either multiplication or comultiplication is invariant under polyadic twist map $\boldsymbol{\tau}_{o p}^{\left(\ell_{\tau}\right)}$ (72), then the querantipode $\boldsymbol{Q}_{\mathrm{id}}$ (218) satisfies

$$
\hat{\boldsymbol{\mu}}_{\star}^{\left(n_{\star}\right)}[\overbrace{\boldsymbol{Q}_{\mathrm{id}}, \ldots, \boldsymbol{Q}_{\mathrm{id}}}^{\left(n_{\star}-1\right)}, \boldsymbol{Q}_{\mathrm{id}} \circ \boldsymbol{Q}_{\mathrm{id}}]=\boldsymbol{Q}_{\mathrm{id}},
$$

where $\boldsymbol{Q}_{\mathrm{id}}^{\circ 2}$ can be on any place, or the convolution querelement (180) of the querantipode $\boldsymbol{Q}_{\mathrm{id}}$ is

$$
\boldsymbol{q}_{\star}\left(\boldsymbol{Q}_{\mathrm{id}}\right)=\boldsymbol{Q}_{\mathrm{id}}^{\circ 2} .
$$

Proof. The proposition follows from applying either (224) or (225) to the l.h.s. of (230), to use (180).

\section{TOWARDS POLYADIC QUANTUM GROUPS}

Bialgebras with a special relaxation of co-commutativity, almost co-commutativity, are the ground objects in the construction of quantum groups identified with the non-commutative and non-co-commutative quasitriangular Hopf algebras $[4,48]$.

\section{Quantum Yang-Baxter equation}

Here we recall the binary case (informally) in a notation that will allow us to provide the "polyadization" in a clearer way.

Let us consider a (binary) bialgebra $B^{(2,2)}=\langle B \mid \mu, \Delta\rangle$, where $\mu=\mu^{(2)}$ is the binary multiplication, $\Delta=\Delta^{(2)}$ (see Definition 98), and the opposite comultiplication is given by $\Delta_{c o p} \equiv \tau_{o p} \circ \Delta$, where $\tau_{o p}$ is the binary twist (66). To relax the co-commutativity condition $\left(\Delta_{\text {cop }}=\Delta\right)$, the following construction inspired by conjugation in groups was proposed [4,48]. A bialgebra $B^{(2,2)}$ is almost co-commutative, if there exists $R \in B \otimes B$ such that (in the elementwise notation)

$$
\mu\left[\Delta_{\text {cop }}(b), R\right]=\mu[R, \Delta(b)], \quad \forall b \in B .
$$

A fixed element $R$ of a bialgebra satisfying (232) is called a universal $R$-matrix. For a co-commutative bialgebra we have $R=e_{B} \otimes e_{B}$, where $e_{B} \in B$ is the unit (element) of the algebra $\langle B \mid \mu\rangle$.

If we demand that $\left\langle B \mid \Delta_{\text {cop }}\right\rangle$ is the opposite coalgebra of $\langle B \mid \Delta\rangle$, and therefore $\Delta_{\text {cop }}$ be coassociative, then $R$ cannot be arbitrary, but has to satisfy some additional conditions, which we will call the almost cocommutativity equations for the $R$-matrix. Indeed, using (232) we can write

$$
\begin{aligned}
& \mu\left[\left(\Delta_{\text {cop }} \otimes \operatorname{id}_{B}\right) \circ \Delta_{\text {cop }}(b), \mu\left[\left(R \otimes e_{B}\right),\left(\Delta \otimes \operatorname{id}_{B}\right)(R)\right]\right] \\
& =\mu\left[\mu\left[\left(R \otimes e_{B}\right),\left(\Delta \otimes \operatorname{id}_{B}\right)(R)\right],\left(\Delta \otimes \operatorname{id}_{B}\right) \circ \Delta(b)\right], \\
& \mu\left[\left(\operatorname{id}_{B} \otimes \Delta_{c o p}\right) \circ \Delta_{c o p}(b), \mu\left[\left(e_{B} \otimes R\right),\left(\operatorname{id}_{B} \otimes \Delta\right)(R)\right]\right] \\
& =\mu\left[\mu\left[\left(e_{B} \otimes R\right),\left(\operatorname{id}_{B} \otimes \Delta\right)(R)\right],\left(\operatorname{id}_{B} \otimes \Delta\right) \circ \Delta(b)\right],
\end{aligned}
$$

Therefore, the coassociativity of $\Delta_{\text {cop }}$ leads to the first almost co-commutativity equation

$$
\mu\left[\left(R \otimes e_{B}\right),\left(\Delta \otimes \operatorname{id}_{B}\right)(R)\right]=\mu\left[\left(e_{B} \otimes R\right),\left(\operatorname{id}_{B} \otimes \Delta\right)(R)\right] .
$$

On the other hand, directly from (232), we have relations which can be treated as the next two almost cocommutativity equations (unconnected to the coassociativity of $\Delta_{c o p}$ )

$$
\begin{aligned}
\mu\left[\left(R \otimes e_{B}\right),\left(\Delta \otimes \operatorname{id}_{B}\right)(R)\right] & =\mu\left[\left(\Delta_{c o p} \otimes \operatorname{id}_{B}\right)(R),\left(R \otimes e_{B}\right)\right] \\
& =\mu\left[\left(\tau_{o p} \otimes \operatorname{id}_{B}\right) \circ\left(\Delta \otimes \operatorname{id}_{B}\right)(R),\left(R \otimes e_{B}\right)\right], \\
\mu\left[\left(e_{B} \otimes R\right),\left(\operatorname{id}_{B} \otimes \Delta\right)(R)\right] & =\mu\left[\left(\operatorname{id}_{B} \otimes \Delta_{c o p}\right)(R),\left(e_{B} \otimes R\right)\right] \\
& =\mu\left[\left(\operatorname{id}_{B} \otimes \tau_{o p}\right) \circ\left(\operatorname{id}_{B} \otimes \Delta\right)(R),\left(e_{B} \otimes R\right)\right] .
\end{aligned}
$$

The equations (235)-(237) for the components of

$$
R=\sum_{\alpha} \mathrm{r}_{\alpha}^{(1)} \otimes \mathrm{r}_{\alpha}^{(2)} \in B \otimes B
$$


are on $B \otimes B \otimes B$. In components the almost co-commutativity (232) can be expressed as follows

$$
\sum_{[b]} \sum_{\alpha} \mu\left[b_{[2]}, r_{\alpha}^{(1)}\right] \otimes \mu\left[b_{[1]}, r_{\alpha}^{(2)}\right]=\sum_{[b]^{\prime}} \sum_{\alpha^{\prime}} \mu\left[r_{\alpha^{\prime}}^{(1)}, b_{[1]^{\prime}}\right] \otimes \mu\left[r_{\alpha^{\prime}}^{(2)}, b_{[2]^{\prime}}\right] .
$$

Now introduce the "extended" form of the $R$-matrix $\mathscr{R}_{i j} \in B \otimes B \otimes B, i, j=1,2,3$, by

$$
\begin{aligned}
& \mathscr{R}_{12}=\sum_{\alpha} \mathrm{r}_{\alpha}^{(1)} \otimes \mathrm{r}_{\alpha}^{(2)} \otimes e_{B} \equiv R \otimes e_{B}, \\
& \mathscr{R}_{13}=\sum_{\alpha} \mathrm{r}_{\alpha}^{(1)} \otimes e_{B} \otimes \mathrm{r}_{\alpha}^{(2)}=\left(\operatorname{id}_{B} \otimes \tau_{o p}\right) \circ\left(R \otimes e_{B}\right), \\
& \mathscr{R}_{23}=\sum_{\alpha} e_{B} \otimes \mathrm{r}_{\alpha}^{(1)} \otimes \mathrm{r}_{\alpha}^{(2)} \equiv e_{B} \otimes R .
\end{aligned}
$$

Obviously, one can try to solve (235)-(237) with respect to the $r_{\alpha}^{(1)}, r_{\alpha}^{(2)}$ directly, but then we are confronted with a difficulty arising from the Sweedler components, because now (see (137)-(139))

$$
\begin{aligned}
\left(\Delta \otimes \operatorname{id}_{B}\right)(R) & =\sum_{\left[\mathrm{r}_{\alpha}^{(1)}\right]} \sum_{\alpha} \mathrm{r}_{\alpha,[1]}^{(1)} \otimes \mathrm{r}_{\alpha,[2]}^{(1)} \otimes \mathrm{r}_{\alpha}^{(2)}, \\
\left(\mathrm{id}_{B} \otimes \Delta\right)(R) & =\sum_{\left[\mathrm{r}_{\alpha}^{(2)}\right]} \sum_{\alpha} \mathrm{r}_{\alpha}^{(1)} \otimes \mathrm{r}_{\alpha,[1]}^{(2)} \otimes \mathrm{r}_{\alpha,[2]}^{(2)} .
\end{aligned}
$$

To avoid computations in the Sweedler components, one can substitute them by the components of $R$ directly as $r_{[j]}^{(i)} \longrightarrow r^{(i)}$ (schematically). This allows us to express (243)-(244) solely through elements of the "extended" $R$-matrix $\mathscr{R}_{i j}$ by

$$
\begin{aligned}
& \left(\Delta \otimes \operatorname{id}_{B}\right)(R)=\mu\left[\mathscr{R}_{13}, \mathscr{R}_{23}\right] \equiv \sum_{\alpha, \beta} \mathrm{r}_{\alpha}^{(1)} \otimes \mathrm{r}_{\beta}^{(1)} \otimes \mu\left[\mathrm{r}_{\alpha}^{(2)}, \mathrm{r}_{\beta}^{(2)}\right], \\
& \left(\operatorname{id}_{B} \otimes \Delta\right)(R)=\mu\left[\mathscr{R}_{13}, \mathscr{R}_{12}\right] \equiv \sum_{\alpha, \beta} \mu\left[\mathrm{r}_{\alpha}^{(1)}, \mathrm{r}_{\beta}^{(1)}\right] \otimes \mathrm{r}_{\beta}^{(2)} \otimes \mathrm{r}_{\alpha}^{(2)},
\end{aligned}
$$

which do not contain Sweedler components of $R$ at all. The equations (245)-(246) define a quasitriangular $R$-matrix [4]. The corresponding almost co-commutative (binary) bialgebra $B_{\text {braid }}^{(2)}=\left\langle B^{(2,2)}, R\right\rangle$ is called a quasitriangular almost co-commutative bialgebra (or braided bialgebra [7]). Only for them can the almost cocommutativity equations (235)-(237) be expressed solely in terms of $R$-matrix components or through the "extended" $R$-matrix $\mathscr{R}_{i j}$, using (245)-(246).

Theorem 119. In the binary case, three almost co-commutativity equations for the R-matrix coincide with

$$
\mu^{\circ 2}\left[\mathscr{R}_{12}, \mathscr{R}_{13}, \mathscr{R}_{23}\right]=\mu^{\circ 2}\left[\mathscr{R}_{23}, \mathscr{R}_{13}, \mathscr{R}_{12}\right] .
$$

Conversely, any quasitriangular $R$-matrix is a solution of (247) by the above construction. The equation for the "extended" $R$-matrix $\mathscr{R}_{i j}(247)$ is called the quantum Yang-Baxter equation $[8,49]$ (or the triangle relation [48]). In terms of the $R$-matrix components (238) the quantum Yang-Baxter equation (247) takes the form

$$
\sum_{\alpha, \beta, \gamma} \mu\left[r_{\alpha}^{(1)}, r_{\beta}^{(1)}\right] \otimes \mu\left[r_{\alpha}^{(2)}, r_{\gamma}^{(1)}\right] \otimes \mu\left[r_{\beta}^{(2)}, r_{\gamma}^{(2)}\right]=\sum_{\alpha^{\prime}, \beta^{\prime}, \gamma^{\prime}} \mu\left[r_{\beta^{\prime}}^{(1)}, r_{\alpha^{\prime}}^{(1)}\right] \otimes \mu\left[r_{\gamma^{\prime}}^{(1)}, r_{\alpha^{\prime}}^{(2)}\right] \otimes \mu\left[r_{\gamma^{\prime}}^{(2)}, r_{\beta^{\prime}}^{(2)}\right]
$$

Let us consider modules over the braided bialgebra $B_{\text {braid }}^{(2,2)}$ and recall [50] how the universal $R$-matrix generalizes the standard flip $\tau_{V_{1} V_{2}}: V_{1} \otimes V_{2} \rightarrow V_{2} \otimes V_{1}$. Define the isomorphism of modules (which in our notation correspond to a 1-place action $\rho(7)) c_{V_{1} V_{2}}: V_{1} \otimes V_{2} \rightarrow V_{2} \otimes V_{1}$ by

$$
\boldsymbol{c}_{V_{1} V_{2}} \circ\left(v_{1} \otimes v_{2}\right)=\boldsymbol{\tau}_{V_{1} V_{2}} \circ R \circ\left(v_{1} \otimes v_{2}\right)=\sum_{\alpha} \rho\left(\mathrm{r}_{\alpha}^{(2)} \mid v_{2}\right) \otimes \rho\left(\mathrm{r}_{\alpha}^{(1)} \mid v_{1}\right), v_{i} \in V_{i}, \mathrm{r}_{\alpha}^{(i)} \in B, i=1,2 .
$$

The quasitriangularity (245)-(246) and (249) on $V_{1} \otimes V_{2} \otimes V_{3}$ leads to (see, e.g., [7])

$$
\begin{aligned}
& \left(\boldsymbol{c}_{V_{1} V_{3}} \otimes \mathrm{id}_{V_{2}}\right) \circ\left(\mathrm{id}_{V_{1}} \otimes \boldsymbol{c}_{V_{2} V_{3}}\right)=\boldsymbol{c}_{V_{1} \otimes V_{2}, V_{3}}, \\
& \left(\mathrm{id}_{V_{2}} \otimes \boldsymbol{c}_{V_{1} V_{3}}\right) \circ\left(\boldsymbol{c}_{V_{1} V_{2}} \otimes \mathrm{id}_{V_{3}}\right)=\boldsymbol{c}_{V_{1}, V_{2} \otimes V_{3}} .
\end{aligned}
$$


Similarly, the quantum Yang-Baxter equation (247) gives the braid equation [50] mapping $V_{1} \otimes V_{2} \otimes V_{3} \rightarrow$ $V_{3} \otimes V_{2} \otimes V_{1}$ :

$$
\left(\boldsymbol{c}_{V_{2} V_{3}} \otimes \mathrm{id}_{V_{1}}\right) \circ\left(\mathrm{id}_{V_{2}} \otimes \boldsymbol{c}_{V_{1} V_{3}}\right) \circ\left(\boldsymbol{c}_{V_{1} V_{2}} \otimes \mathrm{id}_{V_{3}}\right)=\left(\operatorname{id}_{V_{3}} \otimes \boldsymbol{c}_{V_{1} V_{2}}\right) \circ\left(\boldsymbol{c}_{V_{1} V_{3}} \otimes \mathrm{id}_{V_{2}}\right) \circ\left(\mathrm{id}_{V_{1}} \otimes \boldsymbol{c}_{V_{2} V_{3}}\right) .
$$

Putting $V_{1}=V_{2}=V_{3}=V$ shows that $\boldsymbol{c}_{V V}$ is a solution of the braid equation (252) for any module $V$, if the $R$ is a solution of the Yang-Baxter equation [7,50].

\section{$n^{\prime}$-ary braid equation}

Let us consider possible higher arity generalizations of the braid equation (252), informally. Introduce the modules $V_{i}$ over the polyadic bialgebra $B^{\left(n^{\prime}, n\right)}$ (Definition 98) by the $r$-place actions $\rho_{V_{i}}^{(r)}\left(b_{1}, \ldots b_{r} \mid v_{i}\right), b_{j} \in B$, $v_{i} \in V_{i}, i=1, \ldots s, j=1, \ldots, r$ (see (47)). Define the following morphisms of modules

$$
c_{V_{1} \ldots V_{n^{\prime}}}: V_{1} \otimes \ldots \otimes V_{n^{\prime}} \rightarrow V_{n^{\prime}} \otimes \ldots \otimes V_{1} .
$$

We use the shorthand notation $\boldsymbol{c}_{V^{n^{\prime}}} \equiv \boldsymbol{c}_{V_{1} \ldots V_{n^{\prime}}}, \mathrm{id}_{V} \equiv \mathrm{id}_{V_{i}}$ and introduce indices manifestly only when it will be needed.

Proposition 120. The $n^{\prime}$-ary braid equation has the form

$$
\begin{aligned}
& (\boldsymbol{c}_{V^{n^{\prime}}} \otimes \overbrace{\mathrm{id}_{V} \otimes \ldots \otimes \mathrm{id}_{V}}^{n^{\prime}-1}) \circ(\mathrm{id}_{V} \otimes \boldsymbol{c}_{V^{n^{\prime}}} \otimes \overbrace{\mathrm{id}_{V} \otimes \ldots \otimes \mathrm{id}_{V}}^{n^{\prime}-2}) \circ \ldots \\
& \circ(\overbrace{\mathrm{id}_{V} \otimes \ldots \otimes \mathrm{id}_{V} \otimes \boldsymbol{c}_{V^{n^{\prime}}}}^{n^{\prime}-1} \circ(\overbrace{V^{n^{\prime}}} \otimes \overbrace{\mathrm{id}_{V} \otimes \ldots \otimes \mathrm{id}_{V}}^{n^{\prime}-1}) \\
& =(\overbrace{\mathrm{id}_{V} \otimes \ldots \otimes \mathrm{id}_{V}}^{n^{\prime}-1} \otimes \boldsymbol{c}_{V^{n^{\prime}}}) \circ(c_{V^{n^{\prime}}} \otimes \overbrace{\mathrm{id}_{V} \otimes \ldots \otimes \mathrm{id}_{V}}^{n^{\prime}-1}) \circ \ldots \\
& \circ(\overbrace{\mathrm{id}_{V} \otimes \ldots \otimes \mathrm{id}_{V}}^{n^{\prime}-2} \otimes \boldsymbol{c}_{V^{n^{\prime}}} \otimes \mathrm{id}_{V}) \circ(\overbrace{\mathrm{id}_{V} \otimes \ldots \otimes \mathrm{id}_{V} \otimes \boldsymbol{c}_{V^{n^{\prime}}}}^{n^{\prime}-1}),
\end{aligned}
$$

where each side consists of $\left(n^{\prime}+1\right)$ brackets with $\left(2 n^{\prime}-1\right)$ multipliers.

Proof. Use the associative quiver technique from [14] (The Post-like quiver in Section 6).

Remark 121. There can be additional equations depending on the concrete values of $n^{\prime}$ which can contain a different number of brackets determined by the corresponding diagram commutation.

Example 122. In case $n^{\prime}=3$ we have the ternary braided equation for $c_{V_{1} V_{2} V_{3}}: V_{1} \otimes V_{2} \otimes V_{3} \rightarrow V_{3} \otimes V_{2} \otimes V_{1}$ on the tensor product of modules $V_{1} \otimes V_{2} \otimes V_{3} \otimes V_{4} \otimes V_{5}$, as

$$
\begin{aligned}
& \left(\boldsymbol{c}_{V_{3} V_{4} V_{5}} \otimes \mathrm{id}_{V_{2}} \otimes \mathrm{id}_{V_{1}}\right) \circ\left(\mathrm{id}_{V_{3}} \otimes \boldsymbol{c}_{V_{2} V_{5} V_{4}} \otimes \mathrm{id}_{V_{1}}\right) \circ\left(\operatorname{id}_{V_{3}} \otimes \mathrm{id}_{V_{2}} \otimes \boldsymbol{c}_{V_{1} V_{4} V_{5}}\right) \circ\left(\boldsymbol{c}_{V_{1} V_{2} V_{3}} \otimes \operatorname{id}_{V_{4}} \otimes \operatorname{id}_{V_{5}}\right)= \\
& \left(\operatorname{id}_{V_{5}} \otimes \operatorname{id}_{V_{4}} \otimes \boldsymbol{c}_{V_{1} V_{2} V_{3}}\right) \circ\left(\boldsymbol{c}_{V_{1} V_{4} V_{5}} \otimes \mathrm{id}_{V_{2}} \otimes \mathrm{id}_{V_{3}}\right) \circ\left(\operatorname{id}_{V_{1}} \otimes \boldsymbol{c}_{V_{2} V_{5} V_{4}} \otimes \operatorname{id}_{V_{3}}\right) \circ\left(\operatorname{id}_{V_{1}} \otimes \operatorname{id}_{V_{2}} \otimes \boldsymbol{c}_{V_{3} V_{4} V_{5}}\right) .
\end{aligned}
$$

The ternary compatibility conditions for $\boldsymbol{c}_{V_{1} V_{2} V_{3}}$ (corresponding to (250)-(251)) are

$$
\begin{aligned}
& \left(\boldsymbol{c}_{V_{1} V_{4} V_{5}} \otimes \mathrm{id}_{V_{2}} \otimes \mathrm{id}_{V_{3}}\right) \circ\left(\mathrm{id}_{V_{1}} \otimes \boldsymbol{c}_{V_{2} V_{5} V_{4}} \otimes \mathrm{id}_{V_{3}}\right) \circ\left(\mathrm{id}_{V_{1}} \otimes \mathrm{id}_{V_{2}} \otimes \boldsymbol{c}_{V_{3} V_{4} V_{5}}\right)=\boldsymbol{c}_{V_{1} \otimes V_{2} \otimes V_{3}, V_{4}, V_{5}}, \\
& \left(\operatorname{id}_{V_{3}} \otimes \boldsymbol{c}_{V_{2} V_{5} V_{4}} \otimes \operatorname{id}_{V_{1}}\right) \circ\left(\operatorname{id}_{V_{3}} \otimes \operatorname{id}_{V_{2}} \otimes \boldsymbol{c}_{V_{1} V_{4} V_{5}}\right) \circ\left(\boldsymbol{c}_{V_{1} V_{2} V_{3}} \otimes \operatorname{id}_{V_{4}} \otimes \operatorname{id}_{V_{5}}\right)=\boldsymbol{c}_{V_{1}, V_{2}, V_{3} \otimes V_{4} \otimes V_{5}} .
\end{aligned}
$$

Now we follow the opposite (to the standard [50]), but consistent way: using the equations (255)-(257) we find polyadic analogs of the corresponding equations for the $R$-matrix and the quasitriangularity conditions (245)-(246), which will fix the comultiplication structure of a polyadic bialgebra $B^{\left(n^{\prime}, n\right)}$.

\section{Polyadic almost co-commutativity}

We will see that the almost co-commutativity equations for the $R$-matrix are more complicated in the polyadic case, because the main condition (232) will have a different form coming from $n$-ary group theory [47]. Indeed, let $G^{(n)}=\left\langle G \mid \mu^{(n)}\right\rangle$ be an $n$-ary group and $H^{\prime}=\left\langle H^{\prime} \mid \mu^{(n)}\right\rangle, H^{\prime \prime}=\left\langle H^{\prime \prime} \mid \mu^{(n)}\right\rangle$ are its $n$-ary subgroups. 
Recall [47] that $H^{\prime}$ and $H^{\prime \prime}$ are semiconjugated in $G^{(n)}$, if there exist $g \in G$, such that $\mu^{(n)}\left[g, h_{1}^{\prime}, \ldots, h_{n-1}^{\prime}\right]=$ $\mu^{(n)}\left[h_{1}^{\prime \prime}, \ldots, h_{n-1}^{\prime \prime}, g\right], h_{i}^{\prime} \in H^{\prime}, h_{i}^{\prime \prime} \in H^{\prime \prime}$, and if $g$ can be on any place, then $H^{\prime}$ and $H^{\prime \prime}$ are conjugated in $G^{(n)}$. Based on this notion and on analogy with (3), we can "polyadize" the almost co-commutativity condition (232) in the following way.

Let $B^{\left(n^{\prime}, n\right)}=\left\langle B \mid \mu^{(n)}, \Delta^{\left(n^{\prime}\right)}\right\rangle$, be a polyadic bialgebra, and the opposite comultiplication $\Delta_{c o p}^{\left(n^{\prime}\right)}=\tau_{o p}^{\left(n^{\prime}\right)} \circ$ $\Delta^{\left(n^{\prime}\right)}$, where $\tau_{o p}^{\left(n^{\prime}\right)}$ is the polyadic twist $(72)$.

Definition 123. A polyadic bialgebra $B^{\left(n^{\prime}, n\right)}$ is called polyadic sequenced almost co-commutative, if there exist fixed $(n-1)$ elements $R_{i}^{\left(n^{\prime}\right)} \in B^{\otimes n^{\prime}}, i=1, \ldots, n-1$, called a polyadic $R$-matrix sequence, such that

$$
\begin{aligned}
& \mu^{(n)}\left[\Delta_{\text {cop }}^{\left(n^{\prime}\right)}(b), R_{1}^{\left(n^{\prime}\right)}, R_{2}^{\left(n^{\prime}\right)}, \ldots, R_{n-1}^{\left(n^{\prime}\right)}\right] \\
& =\mu^{(n)}\left[R_{1}^{\left(n^{\prime}\right)}, \Delta^{\left(n^{\prime}\right)}(b), R_{2}^{\left(n^{\prime}\right)}, \ldots, R_{n-1}^{\left(n^{\prime}\right)}\right] \\
& \vdots \\
& =\mu^{(n)}\left[R_{1}^{\left(n^{\prime}\right)}, R_{2}^{\left(n^{\prime}\right)}, \ldots, R_{n-1}^{\left(n^{\prime}\right)}, \Delta^{\left(n^{\prime}\right)}(b)\right], \quad \forall b \in B .
\end{aligned}
$$

Definition 124. A polyadic bialgebra $B^{\left(n^{\prime}, n\right)}$ is called polyadic sequenced almost semico-commutative, if only the first and the last relations in (258) hold

$$
\mu^{(n)}\left[\Delta_{c o p}^{\left(n^{\prime}\right)}(b), R_{1}^{\left(n^{\prime}\right)}, R_{2}^{\left(n^{\prime}\right)}, \ldots, R_{n-1}^{\left(n^{\prime}\right)}\right]=\mu^{(n)}\left[R_{1}^{\left(n^{\prime}\right)}, R_{2}^{\left(n^{\prime}\right)}, \ldots, R_{n-1}^{\left(n^{\prime}\right)}, \Delta^{\left(n^{\prime}\right)}(b)\right], \quad \forall b \in B
$$

Remark 125. Using $(n-1)$ polyadic $R$-matrices $R_{i}^{\left(n^{\prime}\right)}$ is the only way to build a polyadic analog for the almost commutativity concept, since now there is no binary multiplication.

The definition (258) is too general and needs to consider $(n-1)$ different polyadic analogs of the $R$-matrix which might not be unique. Therefore, in a similar way to the correspondence of the neutral sequence (3) and the polyadic unit (2), we arrive at

Definition 126. A polyadic bialgebra $B^{\left(n^{\prime}, n\right)}=\left\langle B \mid \mu^{(n)}, \Delta^{\left(n^{\prime}\right)}\right\rangle$ is called polyadic almost (semi)co-commutative, if there exists one fixed element $R^{\left(n^{\prime}\right)} \in B^{\otimes n^{\prime}}$ called a $n^{\prime}$-ary $R$-matrix, such that

$$
\mu^{(n)}[\Delta_{\text {cop }}^{\left(n^{\prime}\right)}(b), \overbrace{R^{\left(n^{\prime}\right)}, \ldots, R^{\left(n^{\prime}\right)}}^{n-1}]=\mu^{(n)}[\overbrace{R^{\left(n^{\prime}\right)}, \ldots, R^{\left(n^{\prime}\right)}}^{n-1}, \Delta^{\left(n^{\prime}\right)}(b)], \quad \forall b \in B .
$$

In components the $n^{\prime}$-ary $R$-matrix $R^{\left(n^{\prime}\right)}$ is

$$
R^{\left(n^{\prime}\right)}=\sum_{\alpha} \mathrm{r}_{\alpha}^{(1)} \otimes \ldots \otimes \mathrm{r}_{\alpha}^{\left(n^{\prime}\right)}, \quad \mathrm{r}_{\alpha}^{(i)} \in B
$$

Remark 127. Polyadic almost co-commutativity (260) can be expressed in component form, as in the binary case (239), only if we know concretely the polyadic twist $\tau_{o p}^{\left(n^{\prime}\right)} \in S_{n^{\prime}}$ (where $S_{n^{\prime}}$ is the symmetry permutation group ON $n^{\prime}$ elements), which is not unique for arbitrary $n^{\prime}>2$.

Example 128. For $B^{(3,3)}$ the ternary almost (semi)co-commutativity (260) is given by

$$
\mu^{(3)}\left[\Delta_{c o p}^{(3)}(b), R^{(3)}, R^{(3)}\right]=\mu^{(3)}\left[R^{(3)}, R^{(3)}, \Delta^{(3)}(b)\right], \quad \forall b \in B,
$$

which with $\tau_{o p}^{(3)}=\left(\begin{array}{l}123 \\ 321\end{array}\right)$ becomes, in components,

$$
\begin{aligned}
& \sum_{[b]} \sum_{\alpha, \beta} \mu^{(3)}\left[b_{[3]}, r_{\alpha}^{(1)}, r_{\beta}^{(1)}\right] \otimes \mu^{(3)}\left[b_{[2]}, r_{\alpha}^{(2)}, r_{\beta}^{(2)}\right] \otimes \mu^{(3)}\left[b_{[1]}, r_{\alpha}^{(3)}, r_{\beta}^{(3)}\right] \\
& =\sum_{[b]^{\prime}} \sum_{\alpha^{\prime} \beta^{\prime}} \mu^{(3)}\left[r_{\alpha^{\prime}}^{(1)}, r_{\beta^{\prime}}^{(1)}, b_{[1]^{\prime}}\right] \otimes \mu^{(3)}\left[r_{\alpha^{\prime}}^{(2)}, r_{\beta^{\prime}}^{(2)}, b_{[2]^{\prime}}\right] \otimes \mu^{(3)}\left[r_{\alpha^{\prime}}^{(3)}, r_{\beta^{\prime}}^{(3)}, b_{[3]^{\prime}}\right] .
\end{aligned}
$$


Example 129. In the exotic mixed case $B^{(4,3)}$ where the polyadic twist "without fixed points" $(72)$ is $\tau_{o p}^{(4)}=\left(\begin{array}{l}1234 \\ 3142\end{array}\right)$, the polyadic almost co-commutativity (260) becomes

$$
\begin{aligned}
& \sum_{[b]} \sum_{\alpha, \beta} \mu^{(3)}\left[b_{[3]}, \mathrm{r}_{\alpha}^{(1)}, \mathrm{r}_{\beta}^{(1)}\right] \otimes \mu^{(3)}\left[b_{[1]}, \mathrm{r}_{\alpha}^{(2)}, \mathrm{r}_{\beta}^{(2)}\right] \otimes \mu^{(3)}\left[b_{[4]}, \mathrm{r}_{\alpha}^{(3)}, \mathrm{r}_{\beta}^{(3)}\right] \otimes \mu^{(3)}\left[b_{[2]}, \mathrm{r}_{\alpha}^{(4)}, \mathrm{r}_{\beta}^{(4)}\right]= \\
& \sum_{[b]^{\prime}} \sum_{\alpha^{\prime} \beta^{\prime}} \mu^{(3)}\left[\mathrm{r}_{\alpha^{\prime}}^{(1)}, \mathrm{r}_{\beta^{\prime}}^{(1)}, b_{[1]^{\prime}}\right] \otimes \mu^{(3)}\left[\mathrm{r}_{\alpha^{\prime}}^{(2)}, \mathrm{r}_{\beta^{\prime}}^{(2)}, b_{[2]^{\prime}}\right] \otimes \mu^{(3)}\left[\mathrm{r}_{\alpha^{\prime}}^{(3)}, \mathrm{r}_{\beta^{\prime}}^{(3)}, b_{[3]^{\prime}}\right] \otimes \mu^{(3)}\left[\mathrm{r}_{\alpha^{\prime}}^{(4)}, \mathrm{r}_{\beta^{\prime}}^{(4)}, b_{[4]^{\prime}}\right] .
\end{aligned}
$$

\section{Equations for the $n^{\prime}$-ary $R$-matrix}

Here we consider the most consistent way (from a categorical viewpoint) to derive equations for the polyadic $R$-matrix, in other words, through using the braided equation (252) (and $n^{\prime}$-ary braided equation (254)) with the concrete choice of the braiding $\boldsymbol{c}_{V^{n^{\prime}}}$.

Suppose that the $n^{\prime}$-ary braiding $\boldsymbol{c}_{V^{n^{\prime}}}$ is defined still by a 1-place action $\rho^{(1)}$, as in the binary case (249). At first glance, we could define the braiding (similar to (249))

$$
\begin{aligned}
& \boldsymbol{c}_{V_{1} \ldots V_{n^{\prime}}} \circ\left(v_{1} \otimes \ldots \otimes v_{n^{\prime}}\right)=\tau_{V_{1} \ldots V_{n^{\prime}}} \circ R^{\left(n^{\prime}\right)} \circ\left(v_{1} \otimes \ldots \otimes v_{n^{\prime}}\right) \\
& =\tau_{V_{1} \ldots V_{n^{\prime}}} \circ\left(\sum_{\alpha} \rho^{(1)}\left(\mathrm{r}_{\alpha}^{(1)} \mid v_{1}\right) \otimes \ldots \otimes \rho^{(1)}\left(\mathrm{r}_{\alpha}^{\left(n^{\prime}\right)} \mid v_{n^{\prime}}\right)\right), \quad v_{i} \in V_{i}, \quad \mathrm{r}_{\alpha}^{(i)} \in B,
\end{aligned}
$$

where $\rho^{(1)}: B \otimes V_{i} \rightarrow V_{i}$ is the 1-place action (see (7)). We recall that only the $n$-ary composition of 1-place actions ( $n$ is the arity of multiplication $\mu^{(n)}$ ) is defined here (see [10])

$$
\overbrace{\rho^{(1)}\left(b_{1} \mid \rho^{(1)}\left(b_{2} \ldots \rho^{(1)}\left(b_{n} \mid v\right)\right)\right)}^{n}=\rho^{(1)}\left(\mu^{(n)}\left[b_{1}, \ldots b_{n}\right] \mid v\right), \quad b_{i} \in B, \quad v \in V .
$$

As in the binary case (240)-(242), we need the "extended" polyadic $R$-matrix.

Remark 130. The standard definition of the "extended" $n^{\prime}$-ary $R$-matrix can be possible, if the algebra $\left\langle B \mid \mu^{(n)}\right\rangle$ contains one polyadic unit (element) $e_{B}$, because in the polyadic case there are new intriguing possibilities (which did not exist in the binary case) of having several units, or even where all elements are units (see the discussion after (3) and [14]).

Definition 131. The "extended" form of the $n^{\prime}$-ary $R$-matrix is defined by $\mathscr{R}_{i_{1} \ldots i_{n^{\prime}}}^{\left(2 n^{\prime}-1\right)} \in B^{\otimes\left(2 n^{\prime}-1\right)}$, such that

$$
\mathscr{R}_{i_{1} \ldots i_{n^{\prime}}}^{\left(2 n^{\prime}-1\right)}=\sum_{\alpha} e_{B} \otimes \ldots \otimes \mathrm{r}_{\alpha}^{\left(i_{1}\right)} \otimes \ldots \otimes \mathrm{r}_{\alpha}^{\left(i_{n^{\prime}}\right)} \otimes \ldots \otimes e_{B}, \quad i_{1}, \ldots, i_{n^{\prime}} \in\left\{1, \ldots 2 n^{\prime}-1\right\}
$$

where $r_{\alpha}^{\left(i_{k}\right)}$ are on the $i_{k}$-place.

In this way we can express in terms of the "extended" $n^{\prime}$-ary $R$-matrix (267) the $n^{\prime}$-ary braided equation (254), in full analogy with the binary case (247).

Example 132. For the ternary case

$$
\begin{aligned}
& \boldsymbol{c}_{V_{1} V_{2} V_{3}} \circ\left(v_{1} \otimes v_{2} \otimes v_{3}\right)=\tau_{V_{1} V_{2} V_{3}} \circ R^{(3)} \circ\left(v_{1} \otimes v_{2} \otimes v_{3}\right) \\
& =\sum_{\alpha} \rho^{(1)}\left(\mathrm{r}_{\alpha}^{(1)} \mid v_{3}\right) \otimes \rho^{(1)}\left(\mathrm{r}_{\alpha}^{(2)} \mid v_{2}\right) \otimes \rho^{(1)}\left(\mathrm{r}_{\alpha}^{(3)} \mid v_{1}\right), \quad v_{i} \in V_{i}, \quad \mathrm{r}_{\alpha}^{(i)} \in B,
\end{aligned}
$$

and we define $\mathscr{R}_{i_{1} i_{2} i_{3}}^{(5)}$ by $(267), i_{1}, i_{2}, i_{3} \in\{1, \ldots, 5\}, \tau_{V_{1} V_{2} V_{3}}=\left(\begin{array}{l}123 \\ 321\end{array}\right)$, and consider the ternary braid equation (255). Using (266) we obtain (informally)

$$
\mathscr{R}_{123}^{(5)} \mathscr{R}_{145}^{(5)} \mathscr{R}_{254}^{(5)} \mathscr{R}_{345}^{(5)}=\mathscr{R}_{345}^{(5)} \mathscr{R}_{254}^{(5)} \mathscr{R}_{145}^{(5)} \mathscr{R}_{123}^{(5)} .
$$

Remark 133. Unfortunately, a "linear" $R^{\left(n^{\prime}\right)} n^{\prime}$-ary braiding $\boldsymbol{c}_{V_{1} \ldots V_{n^{\prime}}}$ (as in (265) and (268)) is not consistent with the polyadic analog of the quasitriangularity equations $(250)-(251)$, because the polyadic almost co-commutativity $(260)$ contains $(n-1)$ copies of $n^{\prime}$-ary $R$-matrix $R^{\left(n^{\prime}\right)}$.

Therefore, in order to agree with (260), instead of (265), we have 
Definition 134. The polyadic braiding $c_{V_{1} \ldots V_{n^{\prime}}}$ is defined by

$$
\begin{aligned}
& \boldsymbol{c}_{V_{1} \ldots V_{n^{\prime}}} \circ\left(v_{1} \otimes \ldots \otimes v_{n^{\prime}}\right)=\tau_{V_{1} \ldots V_{n^{\prime}}} \circ \rho^{(n-1)}(\overbrace{R^{\left(n^{\prime}\right)}, \ldots, R^{\left(n^{\prime}\right)}}^{n-1} \mid\left(v_{1} \otimes \ldots \otimes v_{n^{\prime}}\right)) \\
& =\tau_{V_{1} \ldots V_{n^{\prime}}} \circ\left(\sum_{\alpha_{1}, \ldots \alpha_{n-1}} \rho^{(n-1)}\left(\mathrm{r}_{\alpha_{1}}^{(1)}, \ldots \mathrm{r}_{\alpha_{n-1}}^{(1)} \mid v_{1}\right) \otimes \ldots \otimes \rho^{(n-1)}\left(r_{\alpha_{1}}^{\left(n^{\prime}\right)}, \ldots \mathrm{r}_{\alpha_{n-1}}^{\left(n^{\prime}\right)} \mid v_{n^{\prime}}\right)\right), \\
& v_{i} \in V_{i}, \quad \mathrm{r}_{\alpha}^{(i)} \in B,
\end{aligned}
$$

where $\rho^{(n-1)}: B^{n-1} \otimes V \rightarrow V$ the $(n-1)$-place action (see (7)).

Remark 135. The twist of the modules $\tau_{V_{1} \ldots V_{n^{\prime}}}$ should be compatible with the polyadic twist $\tau_{o p}^{\left(n^{\prime}\right)}$ in (258). In the binary case they are both the same flip $\left(\begin{array}{l}12 \\ 21\end{array}\right)$, but in the $n^{\prime}$-ary case they can be different.

Example 136. Consider the ternary braided equation (255), but now for the braiding $\boldsymbol{c}_{V_{1} V_{2} V_{3}}$, instead of (268), where we have

$$
\begin{aligned}
& \boldsymbol{c}_{V_{1} V_{2} V_{3}} \circ\left(v_{1} \otimes v_{2} \otimes v_{3}\right)=\tau_{V_{1} V_{2} V_{3}} \circ \rho^{(2)}\left(R^{(3)}, R^{(3)} \mid\left(v_{1} \otimes v_{2} \otimes v_{3}\right)\right) \\
& =\sum_{\alpha, \beta} \rho^{(2)}\left(\mathrm{r}_{\alpha}^{(3)}, \mathrm{r}_{\beta}^{(3)} \mid v_{3}\right) \otimes \rho^{(2)}\left(\mathrm{r}_{\alpha}^{(2)}, \mathrm{r}_{\beta}^{(2)} \mid v_{2}\right) \otimes \rho^{(2)}\left(\mathrm{r}_{\alpha}^{(1)}, \mathrm{r}_{\beta}^{(1)} \mid v_{1}\right), \quad v_{i} \in V_{i}, \quad \mathrm{r}_{\alpha, \beta}^{(i)} \in B,
\end{aligned}
$$

where $\rho^{(2)}: B \otimes B \otimes V \rightarrow V$ is a 2-place action (7). In this way (271) is consistent with (262). In each place of the 2-place action $\rho^{(2)}$ we then obtain the relation (269).

\section{Polyadic triangularity}

A polyadic analog of triangularity [4] can be defined, if we rewrite (246) as

$$
\left(\operatorname{id}_{B} \otimes \Delta\right)(R)=\mu\left[\mathscr{R}_{13}, \mathscr{R}_{12}\right] \equiv \sum_{\alpha, \beta} \mu \circ \tau_{o p}\left[\mathrm{r}_{\alpha}^{(1)}, \mathrm{r}_{\beta}^{(1)}\right] \otimes \mathrm{r}_{\alpha}^{(2)} \otimes \mathrm{r}_{\beta}^{(2)}
$$

where $\tau_{o p}$ is the binary twist. Instead of the $R$-matrix formulation (the left equality in (272)), we use the component approach by [3], and propose the following

Definition 137. A polyadic almost co-commutative bialgebra $B^{\left(n^{\prime}, n\right)}=\left\langle B \mid \mu^{(n)}, \Delta^{\left(n^{\prime}\right)}\right\rangle$ with the polyadic $R$-matrix $R^{\left(n^{\prime}\right)}=\sum_{\alpha} \mathrm{r}_{\alpha}^{(1)} \otimes \ldots \otimes \mathrm{r}_{\alpha}^{\left(n^{\prime}\right)}, \quad \mathrm{r}_{\alpha}^{(i)} \in B$ is called quasipolyangular, if the following $n^{\prime}$ relations hold

$$
\begin{gathered}
\sum_{\alpha} \Delta^{\left(n^{\prime}\right)}\left(\mathrm{r}_{\alpha}^{(1)}\right) \otimes \mathrm{r}_{\alpha}^{(2)} \otimes \ldots \otimes \mathrm{r}_{\alpha}^{\left(n^{\prime}\right)}=\sum_{\alpha_{1}, \ldots \alpha_{n^{\prime}}} \mathrm{r}_{\alpha_{1}}^{(1)} \otimes \mathrm{r}_{\alpha_{2}}^{(1)} \otimes \ldots \otimes \mathrm{r}_{\alpha_{n^{\prime}}}^{(1)} \\
\otimes\left(\mu^{(n)}\right)^{\circ \ell}\left[\mathrm{r}_{\alpha_{1}}^{(2)} \otimes \mathrm{r}_{\alpha_{2}}^{(2)} \otimes \ldots \otimes \mathrm{r}_{\alpha_{n^{\prime}}}^{(2)}\right] \otimes \ldots \otimes\left(\mu^{(n)}\right)^{\circ \ell}\left[\mathrm{r}_{\alpha_{1}}^{\left(n^{\prime}\right)} \otimes \mathrm{r}_{\alpha_{2}}^{\left(n^{\prime}\right)} \otimes \ldots \otimes \mathrm{r}_{\alpha_{n^{\prime}}}^{\left(n^{\prime}\right)}\right] \\
\sum_{\alpha} \mathrm{r}_{\alpha}^{(1)} \otimes \Delta^{\left(n^{\prime}\right)}\left(\mathrm{r}_{\alpha}^{(2)}\right) \otimes \ldots \otimes \mathrm{r}_{\alpha}^{\left(n^{\prime}\right)}=\sum_{\alpha_{1}, \ldots \alpha_{n^{\prime}}}\left(\mu^{(n)}\right)^{\alpha \ell} \circ \tau_{o p}^{\left(n^{\prime}\right)}\left[\mathrm{r}_{\alpha_{1}}^{(1)} \otimes \mathrm{r}_{\alpha_{2}}^{(1)} \otimes \ldots \otimes \mathrm{r}_{\alpha_{n^{\prime}}}^{(1)}\right] \\
\otimes \mathrm{r}_{\alpha_{1}}^{(2)} \otimes \mathrm{r}_{\alpha_{2}}^{(2)} \otimes \ldots \otimes \mathrm{r}_{\alpha_{n^{\prime}}}^{(2)} \otimes \ldots \otimes\left(\mu^{(n)}\right)^{\circ \ell}\left[\mathrm{r}_{\alpha_{1}}^{\left(n^{\prime}\right)} \otimes \mathrm{r}_{\alpha_{2}}^{\left(n^{\prime}\right)} \otimes \ldots \otimes \mathrm{r}_{\alpha_{n^{\prime}}}^{\left(n^{\prime}\right)}\right] \\
\sum_{\alpha} \mathrm{r}_{\alpha}^{(1)} \otimes \mathrm{r}_{\alpha}^{(2)} \otimes \ldots \otimes \Delta^{\left(n^{\prime}\right)}\left(\mathrm{r}_{\alpha}^{\left(n^{\prime}\right)}\right)=\sum_{\alpha_{1}, \ldots \alpha_{n^{\prime}}}\left(\mu^{(n)}\right)^{\alpha \ell} \circ \tau_{o p}^{\left(n^{\prime}\right)}\left[\mathrm{r}_{\alpha_{1}}^{(1)} \otimes \mathrm{r}_{\alpha_{2}}^{(1)} \otimes \ldots \otimes \mathrm{r}_{\alpha_{n^{\prime}}}^{(1)}\right] \\
\otimes\left(\mu^{(n)}\right)^{\alpha \ell} \circ \tau_{o p}^{\left(n^{\prime}\right)}\left[\mathrm{r}_{\alpha_{1}}^{(2)} \otimes \mathrm{r}_{\alpha_{2}}^{(2)} \otimes \ldots \otimes \mathrm{r}_{\alpha_{n^{\prime}}}^{(2)}\right] \otimes \ldots \otimes \mathrm{r}_{\alpha_{1}}^{\left(n^{\prime}\right)} \otimes \mathrm{r}_{\alpha_{2}}^{\left(n^{\prime}\right)} \otimes \ldots \otimes \mathrm{r}_{\alpha_{n^{\prime}}}^{\left(n^{\prime}\right)},
\end{gathered}
$$

where $\tau_{o p}^{\left(n^{\prime}\right)}$ is the polyadic twist map (72). The arity shape of a quasipolyangular $B^{\left(n^{\prime}, n\right)}$ is fixed by

$$
n^{\prime}=\ell(n-1)+1, \quad \ell \in \mathbb{N} .
$$


Remark 138. As opposed to the binary case (245)-(246), the right hand sides here can be expressed in terms of the extended $R$-matrix in the first equation (273) and the last one (275) only, because in the intermediate equations the sequences of $R$-matrix elements are permuted. For instance, it is clear that the binary product $\sum_{\alpha, \beta}\left(r_{\beta}^{(1)} \otimes r_{\alpha}^{(2)} \otimes e_{B}\right) \cdot\left(r_{\alpha}^{(1)} \otimes r_{\beta}^{(2)} \otimes e_{B}\right)=\sum_{\alpha, \beta}\left(r_{\beta}^{(1)} \cdot r_{\alpha}^{(1)} \otimes r_{\alpha}^{(2)} \cdot r_{\beta}^{(2)} \otimes e_{B}\right)$ cannot be expressed in terms of the extended binary $R$-matrix (240).

\section{Almost co-medial polyadic bialgebras}

The previous considerations showed that co-commutativity and almost co-commutativity in the polyadic case are not unique and do not describe the bialgebras to the fullest extent. This happens because mediality is a more general and consequent property of polyadic algebraic structures, while commutativity can be treated as a particular case of it (see (66)). Therefore, we propose here to deform co-mediality (rather than co-commutativity as in $[4,48,50])$.

Let $B^{\left(n^{\prime}, n\right)}=\left\langle B \mid \mu^{(n)}, \Delta^{\left(n^{\prime}\right)}\right\rangle$, be a polyadic bialgebra. Now we deform the co-mediality condition (120) in a similar way to the polyadic $R$-matrix (260).

Definition 139. A polyadic bialgebra $B^{\left(n^{\prime}, n\right)}$ is called polyadic sequenced almost co-medial, if there exist $\left(n^{\prime}-1\right)$ fixed elements $M_{i}^{\left(n^{\prime 2}\right)} \in B^{\otimes n^{\prime 2}}, i=1, \ldots, n-1$, called a polyadic $M$-matrix sequence, such that (see (120) and (260))

$$
\begin{aligned}
& \mu^{(n)}\left[\tau_{\text {medial }}^{\left(n^{\prime}, n^{\prime}\right)} \circ\left(\left(\Delta^{\left(n^{\prime}\right)}\right)^{\otimes n^{\prime}}\right) \circ \Delta^{\left(n^{\prime}\right)}(b), M_{1}^{\left(n^{\prime 2}\right)}, M_{2}^{\left(n^{\prime 2}\right)}, \ldots, M_{n-1}^{\left(n^{\prime 2}\right)}\right] \\
& =\mu^{(n)}\left[M_{1}^{\left(n^{\prime 2}\right)},\left(\left(\Delta^{\left(n^{\prime}\right)}\right)^{\otimes n^{\prime}}\right) \circ \Delta^{\left(n^{\prime}\right)}(b), M_{2}^{\left(n^{\prime 2}\right)}, \ldots, M_{n-1}^{\left(n^{\prime 2}\right)}\right] \\
& =\mu^{(n)}\left[M_{1}^{\left(n^{\prime 2}\right)}, M_{2}^{\left(n^{\prime 2}\right)}, \ldots, M_{n-1}^{\left(n^{\prime 2}\right)},\left(\left(\Delta^{\left(n^{\prime}\right)}\right)^{\otimes n^{\prime}}\right) \circ \Delta^{\left(n^{\prime}\right)}(b)\right], \quad \forall b \in B,
\end{aligned}
$$

where $\boldsymbol{\tau}_{\text {medial }}^{\left(n^{\prime}, n^{\prime}\right)}$ is the polyadic medial map (69).

Definition 140. A polyadic bialgebra $B^{\left(n^{\prime}, n\right)}$ is called polyadic sequenced almost (semi)co-medial, if only the first and the last relations in (277) hold

$$
\begin{aligned}
& \mu^{(n)}\left[\tau_{\text {medial }}^{\left(n^{\prime}, n^{\prime}\right)} \circ\left(\left(\Delta^{\left(n^{\prime}\right)}\right)^{\otimes n^{\prime}}\right) \circ \Delta^{\left(n^{\prime}\right)}(b), M_{1}^{\left(n^{\prime 2}\right)}, \ldots, M_{n-1}^{\left(n^{\prime 2}\right)}\right] \\
& =\mu^{(n)}\left[M_{1}^{\left(n^{\prime 2}\right)}, \ldots, M_{n-1}^{\left(n^{\prime 2}\right)},\left(\left(\Delta^{\left(n^{\prime}\right)}\right)^{\otimes n^{\prime}}\right) \circ \Delta^{\left(n^{\prime}\right)}(b)\right], \quad \forall b \in B,
\end{aligned}
$$

If all the elements in the sequence (similar to the neutral sequence for $n$-ary groups $(3)$ ) are the same $M_{1}^{\left(n^{\prime 2}\right)}=M_{2}^{\left(n^{\prime 2}\right)}=\ldots=M_{n-1}^{\left(n^{\prime 2}\right)} \equiv M^{\left(n^{\prime 2}\right)}$, we have

Definition 141. A polyadic bialgebra $B^{\left(n^{\prime}, n\right)}$ is called polyadic almost (semi)co-medial, if there exist one fixed element $M^{\left(n^{\prime 2}\right)} \in B^{\otimes n^{\prime 2}}$ called a polyadic $M$-matrix, such that (see (120) and (260))

$$
\begin{aligned}
& \mu^{(n)}[\tau_{\text {medial }}^{\left(n^{\prime}, n^{\prime}\right)} \circ\left(\left(\Delta^{\left(n^{\prime}\right)}\right)^{\otimes n^{\prime}}\right) \circ \Delta^{\left(n^{\prime}\right)}(b), \overbrace{M^{\left(n^{\prime 2}\right)}, \ldots, M^{\left(n^{\prime 2}\right)}}^{n-1}] \\
& =\mu^{(n)}[\overbrace{M^{\left(n^{\prime 2}\right)}, \ldots, M^{\left(n^{\prime 2}\right)}}^{n-1},\left(\left(\Delta^{\left(n^{\prime}\right)}\right)^{\otimes n^{\prime}}\right) \circ \Delta^{\left(n^{\prime}\right)}(b)], \quad \forall b \in B .
\end{aligned}
$$

Remark 142. The main advantage of the polyadic almost co-mediality property over polyadic almost cocommutativity is the uniqueness of the medial map $\boldsymbol{\tau}_{\text {medial }}^{(n, n)}$ and nonuniqueness of the polyadic twist map $\tau_{o p}^{\left(\ell_{\tau}\right)}(72)$

The polyadic $M$-matrix $M^{\left(n^{\prime}\right)}$ in components is given by

$$
M^{\left(n^{\prime 2}\right)}=\sum_{\alpha} \mathrm{m}_{\alpha}^{(1)} \otimes \ldots \otimes \mathrm{m}_{\alpha}^{\left(n^{\prime 2}\right)}, \quad \mathrm{m}_{\alpha}^{(i)} \in B, \quad i=1, \ldots, n^{\prime 2} .
$$


Example 143. In the binary case for $B^{(2,2)}=\langle B \mid \mu, \Delta\rangle$ we have an almost co-mediality (279) as

$$
\mu\left[\tau_{\text {medial }} \circ(\Delta \otimes \Delta) \circ \Delta(b), M^{(4)}\right]=\mu\left[M^{(4)},(\Delta \otimes \Delta) \circ \Delta(b)\right], \quad \forall b \in B .
$$

which gives, in components (cf. for $R$-matrix (239))

$$
\begin{aligned}
& \sum_{[b]_{[b]}} \sum_{\alpha} \mu\left[b_{[1]_{[1]}}, \mathbf{m}_{\alpha}^{(1)}\right] \otimes \mu\left[b_{[2]_{[1]}}, \mathbf{m}_{\alpha}^{(2)}\right] \otimes \mu\left[b_{[1]_{[2]}}, \mathbf{m}_{\alpha}^{(3)}\right] \otimes \mu\left[b_{[2]_{[2]}}, \mathbf{m}_{\alpha}^{(4)}\right] \\
& =\sum_{[b]_{[b]}^{\prime}} \sum_{\alpha^{\prime}} \mu\left[\mathrm{m}_{\alpha^{\prime}}^{(1)}, b_{\left.[1]_{[1]}^{\prime}\right]}\right] \otimes \mu\left[\mathrm{m}_{\alpha^{\prime}}^{(2)}, b_{\left.[1]_{[2]}^{\prime}\right]}\right] \otimes \mu\left[\mathrm{m}_{\alpha^{\prime}}^{(3)}, b_{[2]_{[1]}^{\prime}}\right] \otimes \mu\left[\mathrm{m}_{\alpha^{\prime}}^{(4)}, b_{\left.[2]_{[2]}^{\prime}\right]}\right] .
\end{aligned}
$$

Let us clarify the connection between the almost co-commutativity and almost co-mediality properties.

Theorem 144. If $B^{\left(n^{\prime}, n\right)}$ is polyadic almost (semi)co-commutative with the polyadic twist map $\tau_{o p}^{\left(n^{\prime}\right)}$ (72) and the $n^{\prime}$-ary R-matrix $R^{\left(n^{\prime}\right)}$ (261), then (260) can be presented in the "medial-like" form

$$
\begin{aligned}
& \mu^{(n)}[\tau_{R}^{\left(n^{\prime}, n^{\prime}\right)} \circ\left(\left(\Delta^{\left(n^{\prime}\right)}\right)^{\otimes n^{\prime}}\right) \circ \Delta^{\left(n^{\prime}\right)}(b), \overbrace{M_{R}^{\left(n^{\prime 2}\right)}, \ldots, M_{R}^{\left(n^{\prime 2}\right)}}^{n-1}] \\
& =\mu^{(n)}[\overbrace{M_{R}^{\left(n^{\prime 2}\right)}, \ldots, M_{R}^{\left(n^{\prime 2}\right)}}^{n-1},\left(\left(\Delta^{\left(n^{\prime}\right)}\right)^{\otimes n^{\prime}}\right) \circ \Delta^{\left(n^{\prime}\right)}(b)], \quad \forall b \in B,
\end{aligned}
$$

where

$$
\begin{aligned}
\tau_{R}^{\left(n^{\prime}, n^{\prime}\right)} & =\overbrace{\tau_{\tau_{o p}}^{\left(n^{\prime}\right)} \otimes \ldots \otimes \tau_{o p}^{\left(n^{\prime}\right)}}^{n^{\prime}}, \\
M_{R}^{\left(n^{\prime 2}\right)} & =\overbrace{R^{\left(n^{\prime}\right)} \otimes \ldots \otimes R^{\left(n^{\prime}\right)}}^{n^{\prime}} .
\end{aligned}
$$

Proof. Applying (260) to each Sweedler component $b_{[i]}$ of $\Delta^{\left(n^{\prime}\right)}(b), i=1, \ldots, n^{\prime}$, we obtain $n^{\prime}$ relations for the polyadic almost (semi)co-commutativity. Then multiplying them tensorially, we obtain

$$
\begin{aligned}
& (\overbrace{\tau_{o p}^{\left(n^{\prime}\right)} \otimes \ldots \otimes \tau_{o p}^{\left(n^{\prime}\right)}}^{n^{\prime}}) \circ\left(\Delta^{\left(n^{\prime}\right)}\left(b_{[1]}\right) \otimes \ldots \otimes \Delta^{\left(n^{\prime}\right)}\left(b_{\left[n^{\prime}\right]}\right)\right) \circ(\overbrace{R^{\left(n^{\prime}\right)} \otimes \ldots \otimes R^{\left(n^{\prime}\right)}}^{n^{\prime}}) \\
& =(\overbrace{R^{\left(n^{\prime}\right) \otimes \ldots \otimes R^{\left(n^{\prime}\right)}}}^{n^{\prime}}) \circ\left(\Delta^{\left(n^{\prime}\right)}\left(b_{[1]}\right) \otimes \ldots \otimes \Delta^{\left(n^{\prime}\right)}\left(b_{\left[n^{\prime}\right]}\right)\right),
\end{aligned}
$$

which immediately gives (283). The converse statement is obvious.

Corollary 145. Polyadic almost co-commutativity is a particular case of polyadic co-mediality with the special "medial-like" twist map $\boldsymbol{\tau}_{R}^{\left(n^{\prime}, n^{\prime}\right)}(284)$ and the composite $M$-matrix (285) consisting of $n^{\prime}$ copies of the $R$-matrix (261).

Example 146. In the binary case we compare the medial map (66) with the composed "medial-like" twist map (284) as

$$
\begin{aligned}
\boldsymbol{\tau}_{\text {medial }} & =\mathrm{id}_{B} \otimes \boldsymbol{\tau}_{o p} \otimes \mathrm{id}_{B} \\
\boldsymbol{\tau}_{R} & =\boldsymbol{\tau}_{o p} \otimes \boldsymbol{\tau}_{o p}
\end{aligned}
$$


or in components

$$
\begin{aligned}
& b_{1} \otimes b_{2} \otimes b_{3} \otimes b_{4} \stackrel{\tau_{\text {medial }}}{\mapsto} b_{1} \otimes b_{3} \otimes b_{2} \otimes b_{4}, \\
& b_{1} \otimes b_{2} \otimes b_{3} \otimes b_{4} \stackrel{\tau_{R}}{\mapsto} b_{2} \otimes b_{1} \otimes b_{4} \otimes b_{3} .
\end{aligned}
$$

This shows manifestly the difference between (polyadic) almost co-commutativity and (polyadic) almost comediality.

\section{Equations for the $M$-matrix}

Let us find the equations for the $M$-matrix (279) using the medial analog of the $n^{\prime}$-ary braid equation. Now the morphism of modules $\boldsymbol{c}_{V_{1} \ldots V_{n^{\prime 2}}}$ becomes (see for the $R$-matrix (265))

$$
\begin{aligned}
& \boldsymbol{c}_{V_{1} \ldots V_{n^{\prime 2}}} \circ\left(v_{1} \otimes \ldots \otimes v_{n^{\prime 2}}\right)=\tau_{\text {medial }, V_{1} \ldots V_{n^{\prime 2}}}^{\left(n^{\prime}, n^{\prime}\right)} \circ \rho^{(n-1)}(\overbrace{M^{\left(n^{\prime 2}\right)}, \ldots, M^{\left(n^{\prime 2}\right)}}^{n-1} \mid\left(v_{1} \otimes \ldots \otimes v_{n^{\prime 2}}\right))= \\
& \tau_{\text {medial }, V_{1} \ldots V_{n^{\prime 2}}}^{\left(n^{\prime}, n^{\prime}\right)} \circ\left(\sum_{\alpha_{1}, \ldots \alpha_{n-1}} \rho^{(n-1)}\left(\mathrm{m}_{\alpha_{1}}^{(1)}, \ldots \mathrm{m}_{\alpha_{n-1}}^{(1)} \mid v_{1}\right) \otimes \ldots \otimes \rho^{(n-1)}\left(\mathrm{m}_{\alpha_{1}}^{\left(n^{\prime 2}\right)}, \ldots \mathrm{m}_{\alpha_{n-1}}^{\left(n^{\prime 2}\right)} \mid v_{n^{\prime 2}}\right)\right), \\
& v_{i} \in V_{i}, \quad \mathrm{r}_{\alpha}^{(i)} \in B, \quad i=1, \ldots, n^{\prime 2},
\end{aligned}
$$

where $\tau_{\text {medial }, V_{1} \ldots V_{n^{\prime 2}}}^{\left(n^{\prime}, n^{\prime}\right)}$ is the medial map (69) acting on $n^{\prime 2} \operatorname{modules} V_{i}, M^{\left(n^{\prime 2}\right)}$ is the polyadic $M$-matrix (280), and $\rho^{(n-1)}$ is the $(n-1)$-place action $(7)$. Now instead of the $n^{\prime}$-ary braid equation (254) we can have

Proposition 147. The $n^{\prime}$-ary medial braid equation is

$$
\begin{aligned}
& (\boldsymbol{c}_{V^{n^{\prime 2}}} \otimes \overbrace{\mathrm{id}_{V} \otimes \ldots \otimes \mathrm{id}_{V}}^{n^{\prime 2}-1}) \circ(\mathrm{id}_{V} \otimes \boldsymbol{c}_{V^{n^{\prime 2}}} \otimes \overbrace{\mathrm{id}_{V} \otimes \ldots \otimes \mathrm{id}_{V}}^{n^{\prime 2}-2}) \circ \ldots \\
& \circ(\overbrace{\mathrm{id}_{V} \otimes \ldots \otimes \mathrm{id}_{V}}^{n^{\prime 2}-1} \otimes c_{V^{n^{\prime 2}}}) \circ(c_{V^{n^{\prime 2}}} \otimes \overbrace{\mathrm{id}_{V} \otimes \ldots \otimes \mathrm{id}_{V}}^{n^{\prime 2}-1}) \\
& =(\overbrace{\mathrm{id}_{V} \otimes \ldots \otimes \mathrm{id}_{V}}^{n^{\prime 2}-1} \otimes c_{V^{n^{\prime 2}}}) \circ(\boldsymbol{c}_{V^{n^{\prime 2}}} \otimes \overbrace{\mathrm{id}_{V} \otimes \ldots \otimes \mathrm{id}_{V}}^{n^{\prime 2}-1}) \circ \ldots \\
& \circ(\overbrace{\mathrm{id}_{V} \otimes \ldots \otimes \mathrm{id}_{V}}^{n^{\prime 2}-2} \otimes c_{V^{n^{\prime 2}}} \otimes \mathrm{id}_{V}) \circ(\overbrace{\mathrm{id}_{V} \otimes \ldots \otimes \mathrm{id}_{V}}^{n^{\prime 2}-1} \otimes c_{V^{n^{\prime 2}}}),
\end{aligned}
$$

where we use the notation $\boldsymbol{c}_{V^{n^{\prime 2}}} \equiv \boldsymbol{c}_{V_{1} \ldots V_{n^{\prime 2}}}, \mathrm{id}_{V} \equiv \mathrm{id}_{V_{i}}$, and each side consists of $\left(n^{\prime 2}+1\right)$ brackets with $\left(2 n^{\prime 2}-1\right)$ multipliers.

Proof. This follows from the associative quiver technique [14].

We observe that even in the binary case the medial braid equations are cumbersome and nontrivial.

Example 148. In the binary case $n^{\prime}=2$ we have the map $\boldsymbol{c}_{V^{n^{\prime 2}}}($ see $(286),(288))$

$$
c_{V_{1} V_{2} V_{3} V_{4}}: V_{1} \otimes V_{2} \otimes V_{3} \otimes V_{4} \rightarrow V_{1} \otimes V_{3} \otimes V_{2} \otimes V_{4}
$$

which acts on

$$
V_{1} \otimes V_{2} \otimes V_{3} \otimes V_{4} \otimes V_{5} \otimes V_{6} \otimes V_{7}
$$

There are two medial braid equations which correspond to diagrams of different lengths (cf. the standard braid equation (252)) 
1) $V_{1} \otimes V_{2} \otimes V_{3} \otimes V_{4} \otimes V_{5} \otimes V_{6} \otimes V_{7} \longrightarrow V_{1} \otimes V_{4} \otimes V_{5} \otimes V_{6} \otimes V_{3} \otimes V_{2} \otimes V_{7}:$

$\left(c_{V_{1} V_{5} V_{4} V_{6}} \otimes \mathrm{id}_{V_{3}} \otimes \mathrm{id}_{V_{2}} \otimes \mathrm{id}_{V_{7}}\right) \circ\left(\mathrm{id}_{V_{1}} \otimes c_{V_{5} V_{6} V_{4} V_{3}} \otimes \mathrm{id}_{V_{2}} \otimes \mathrm{id}_{V_{7}}\right)$

$\circ\left(\mathrm{id}_{V_{1}} \otimes \mathrm{id}_{V_{5}} \otimes \boldsymbol{c}_{V_{6} V_{3} V_{4} V_{2}} \otimes \mathrm{id}_{V_{7}}\right) \circ\left(\mathrm{id}_{V_{1}} \otimes \mathrm{id}_{V_{5}} \otimes \mathrm{id}_{V_{6}} \otimes \boldsymbol{c}_{V_{3} V_{2} V_{4} V_{7}}\right)$

$\circ\left(c_{V_{1} V_{6} V_{5} V_{3}} \otimes \mathrm{id}_{V_{2}} \otimes \mathrm{id}_{V_{4}} \otimes \mathrm{id}_{V_{7}}\right) \circ\left(\mathrm{id}_{V_{1}} \otimes c_{V_{6} V_{3} V_{5} V_{2}} \otimes \mathrm{id}_{V_{4}} \otimes \mathrm{id}_{V_{7}}\right)$

$\circ\left(\mathrm{id}_{V_{1}} \otimes \mathrm{id}_{V_{6}} \otimes \boldsymbol{c}_{V_{3} V_{2} V_{5} V_{4}} \otimes \mathrm{id}_{V_{7}}\right) \circ\left(\mathrm{id}_{V_{1}} \otimes \mathrm{id}_{V_{6}} \otimes \mathrm{id}_{V_{6}} \otimes \boldsymbol{c}_{V_{2} V_{4} V_{5} V_{7}}\right)$

$\circ\left(\boldsymbol{c}_{V_{1} V_{3} V_{6} V_{2}} \otimes \mathrm{id}_{V_{4}} \otimes \mathrm{id}_{V_{5}} \otimes \mathrm{id}_{V_{7}}\right) \circ\left(\mathrm{id}_{V_{1}} \otimes \boldsymbol{c}_{V_{3} V_{2} V_{6} V_{4}} \otimes \mathrm{id}_{V_{5}} \otimes \mathrm{id}_{V_{7}}\right)$

$\circ\left(\mathrm{id}_{V_{1}} \otimes \mathrm{id}_{V_{3}} \otimes \boldsymbol{c}_{V_{2} V_{4} V_{6} V_{5}} \otimes \mathrm{id}_{V_{7}}\right) \circ\left(\mathrm{id}_{V_{1}} \otimes \mathrm{id}_{V_{3}} \otimes \mathrm{id}_{V_{2}} \otimes \boldsymbol{c}_{V_{4} V_{5} V_{6} V_{7}}\right)$

$\circ\left(c_{V_{1} V_{2} V_{3} V_{4}} \otimes \mathrm{id}_{V_{5}} \otimes \mathrm{id}_{V_{6}} \otimes \mathrm{id}_{V_{7}}\right)=\left(\mathrm{id}_{V_{1}} \otimes \mathrm{id}_{V_{4}} \otimes \mathrm{id}_{V_{5}} \otimes c_{V_{6} V_{2} V_{3} V_{7}}\right)$

$\circ\left(\boldsymbol{c}_{V_{1} V_{5} V_{4} V_{6}} \otimes \mathrm{id}_{V_{2}} \otimes \mathrm{id}_{V_{3}} \otimes \mathrm{id}_{V_{7}}\right) \circ\left(\mathrm{id}_{V_{1}} \otimes \boldsymbol{c}_{V_{5} V_{6} V_{4} V_{2}} \otimes \operatorname{id}_{V_{3}} \otimes \mathrm{id}_{V_{7}}\right)$

$\circ\left(\mathrm{id}_{V_{1}} \otimes \mathrm{id}_{V_{5}} \otimes \boldsymbol{c}_{V_{6} V_{2} V_{4} V_{3}} \otimes \mathrm{id}_{V_{7}}\right) \circ\left(\mathrm{id}_{V_{1}} \otimes \mathrm{id}_{V_{5}} \otimes \mathrm{id}_{V_{6}} \otimes \boldsymbol{c}_{V_{2} V_{3} V_{4} V_{7}}\right)$

$\circ\left(c_{V_{1} V_{6} V_{5} V_{2}} \otimes \mathrm{id}_{V_{3}} \otimes \mathrm{id}_{V_{4}} \otimes \mathrm{id}_{V_{7}}\right) \circ\left(\mathrm{id}_{V_{1}} \otimes c_{V_{6} V_{2} V_{5} V_{3}} \otimes \mathrm{id}_{V_{4}} \otimes \mathrm{id}_{V_{7}}\right)$

$\circ\left(\mathrm{id}_{V_{1}} \otimes \mathrm{id}_{V_{6}} \otimes \boldsymbol{c}_{V_{2} V_{3} V_{5} V_{4}} \otimes \mathrm{id}_{V_{7}}\right) \circ\left(\mathrm{id}_{V_{1}} \otimes \mathrm{id}_{V_{6}} \otimes \mathrm{id}_{V_{2}} \otimes \boldsymbol{c}_{V_{3} V_{4} V_{5} V_{7}}\right)$

$\circ\left(c_{V_{1} V_{2} V_{6} V_{3}} \otimes \mathrm{id}_{V_{4}} \otimes \mathrm{id}_{V_{5}} \otimes \mathrm{id}_{V_{7}}\right) \circ\left(\mathrm{id}_{V_{1}} \otimes c_{V_{6} V_{3} V_{5} V_{2}} \otimes \mathrm{id}_{V_{4}} \otimes \mathrm{id}_{V_{7}}\right)$

$\circ\left(\mathrm{id}_{V_{1}} \otimes \mathrm{id}_{V_{2}} \otimes \boldsymbol{c}_{V_{3} V_{4} V_{6} V_{5}} \otimes \mathrm{id}_{V_{7}}\right) \circ\left(\mathrm{id}_{V_{1}} \otimes \mathrm{id}_{V_{2}} \otimes \mathrm{id}_{V_{3}} \otimes \boldsymbol{c}_{V_{4} V_{5} V_{6} V_{7}}\right)$.

2) $V_{1} \otimes V_{2} \otimes V_{3} \otimes V_{4} \otimes V_{5} \otimes V_{6} \otimes V_{7} \longrightarrow V_{1} \otimes V_{6} \otimes V_{5} \otimes V_{4} \otimes V_{2} \otimes V_{3} \otimes V_{7}$ :

$\left(\mathrm{id}_{V_{1}} \otimes \mathrm{id}_{V_{6}} \otimes \boldsymbol{c}_{V_{5} V_{2} V_{4} V_{3}} \otimes \mathrm{id}_{V_{7}}\right) \circ\left(\mathrm{id}_{V_{1}} \otimes \mathrm{id}_{V_{6}} \otimes \mathrm{id}_{V_{5}} \otimes c_{V_{2} V_{3} V_{4} V_{7}}\right)$

$\circ\left(\boldsymbol{c}_{V_{1} V_{5} V_{6} V_{2}} \otimes \operatorname{id}_{V_{3}} \otimes \operatorname{id}_{V_{4}} \otimes \operatorname{id}_{V_{7}}\right) \circ\left(\operatorname{id}_{V_{1}} \otimes c_{V_{5} V_{2} V_{6} V_{3}} \otimes \operatorname{id}_{V_{4}} \otimes \operatorname{id}_{V_{7}}\right)$

$\circ\left(\mathrm{id}_{V_{1}} \otimes \mathrm{id}_{V_{5}} \otimes \boldsymbol{c}_{V_{2} V_{3} V_{6} V_{4}} \otimes \mathrm{id}_{V_{7}}\right) \circ\left(\mathrm{id}_{V_{1}} \otimes \mathrm{id}_{V_{5}} \otimes \mathrm{id}_{V_{2}} \otimes \boldsymbol{c}_{V_{3} V_{4} V_{6} V_{7}}\right)$

$\circ\left(c_{V_{1} V_{2} V_{5} V_{3}} \otimes \mathrm{id}_{V_{4}} \otimes \mathrm{id}_{V_{6}} \otimes \mathrm{id}_{V_{7}}\right) \circ\left(\mathrm{id}_{V_{1}} \otimes c_{V_{2} V_{3} V_{5} V_{4}} \otimes \mathrm{id}_{V_{6}} \otimes \mathrm{id}_{V_{7}}\right)$

$\circ\left(\mathrm{id}_{V_{1}} \otimes \mathrm{id}_{V_{2}} \otimes \boldsymbol{c}_{V_{3} V_{4} V_{5} V_{6}} \otimes \mathrm{id}_{V_{7}}\right)=\left(\mathrm{id}_{V_{1}} \otimes c_{V_{6} V_{4} V_{5} V_{2}} \otimes \mathrm{id}_{V_{3}} \otimes \mathrm{id}_{V_{7}}\right)$

$\circ\left(\mathrm{id}_{V_{1}} \otimes \mathrm{id}_{V_{6}} \otimes \boldsymbol{c}_{V_{4} V_{2} V_{5} V_{3}} \otimes \mathrm{id}_{V_{7}}\right) \circ\left(\mathrm{id}_{V_{1}} \otimes \mathrm{id}_{V_{6}} \otimes \mathrm{id}_{V_{4}} \otimes \boldsymbol{c}_{V_{2} V_{3} V_{5} V_{7}}\right)$

$\circ\left(c_{V_{1} V_{4} V_{6} V_{2}} \otimes \mathrm{id}_{V_{3}} \otimes \mathrm{id}_{V_{5}} \otimes \mathrm{id}_{V_{7}}\right) \circ\left(\mathrm{id}_{V_{1}} \otimes \boldsymbol{c}_{V_{4} V_{2} V_{6} V_{3}} \otimes \mathrm{id}_{V_{5}} \otimes \mathrm{id}_{V_{7}}\right)$

$\circ\left(\mathrm{id}_{V_{1}} \otimes \mathrm{id}_{V_{4}} \otimes \boldsymbol{c}_{V_{2} V_{3} V_{6} V_{5}} \otimes \mathrm{id}_{V_{7}}\right) \circ\left(\mathrm{id}_{V_{1}} \otimes \mathrm{id}_{V_{4}} \otimes \mathrm{id}_{V_{2}} \otimes \boldsymbol{c}_{V_{3} V_{5} V_{6} V_{7}}\right)$

$\circ\left(\boldsymbol{c}_{V_{1} V_{2} V_{4} V_{3}} \otimes \mathrm{id}_{V_{5}} \otimes \mathrm{id}_{V_{6}} \otimes \mathrm{id}_{V_{7}}\right) \circ\left(\mathrm{id}_{V_{1}} \otimes c_{V_{2} V_{3} V_{4} V_{5}} \otimes \operatorname{id}_{V_{6}} \otimes \operatorname{id}_{V_{7}}\right)$.

The equations for the $M$-matrix can be obtained by introducing the "extended" $M$-matrix, as in the case of the $R$-matrix, and this can also be possible if the $n$-ary algebra $\left\langle B \mid \mu^{(n)}\right\rangle$ has the unit (element) $e_{B} \in B$.

Definition 149. The "extended" $M$-matrix is defined by $\mathscr{M}_{i_{1} \ldots i_{n^{\prime 2}}}^{\left(2 n^{\prime 2}-1\right)} \in B^{\otimes\left(2 n^{\prime 2}-1\right)}$, such that

$$
\mathcal{M}_{i_{1} \ldots i_{n^{\prime 2}}}^{\left(2 n^{\prime 2}-1\right)}=\sum_{\alpha} e_{B} \otimes \ldots \otimes \mathrm{m}_{\alpha}^{\left(i_{1}\right)} \otimes \ldots \otimes \mathrm{m}_{\alpha}^{\left(i_{n^{\prime 2}}\right)} \otimes \ldots \otimes e_{B}, \quad i_{1}, \ldots, i_{n^{\prime 2}} \in\left\{1, \ldots, 2 n^{\prime 2}-1\right\}
$$

where $\mathrm{m}_{\alpha}^{\left(i_{k}\right)}$ are on the $i_{k}$-place.

It is difficult to write the general compatibility equations for the "extended" $M$-matrix (295).

Example 150. In the binary case $n=n^{\prime}=2$ we have for the polyadic $M$-matrix $M^{(4)}$ in components

$$
M^{(4)}=\sum_{\alpha} \mathrm{m}_{\alpha}^{(1)} \otimes \mathrm{m}_{\alpha}^{(2)} \otimes \mathrm{m}_{\alpha}^{(3)} \otimes \mathrm{m}_{\alpha}^{(4)}, \quad \mathrm{m}_{\alpha}^{(i)} \in B,
$$

and $\mathscr{M}_{i_{1} \ldots i_{4}}^{(7)} \in B^{\otimes 7}$ with

$$
\mathscr{M}_{i_{1} \ldots i_{4}}^{(7)}=\sum_{\alpha} e_{B} \otimes \ldots \otimes \mathrm{m}_{\alpha}^{\left(i_{1}\right)} \otimes \ldots \otimes \mathrm{m}_{\alpha}^{\left(i_{4}\right)} \otimes \ldots \otimes e_{B}, \quad i_{1}, \ldots, i_{4} \in\{1, \ldots, 7\} .
$$

The map of modules $\boldsymbol{c}_{V_{1} \ldots V_{4}}$ (292) in the manifest form is

$$
\begin{aligned}
& \boldsymbol{c}_{V_{1} V_{2} V_{3} V_{4}} \circ\left(v_{1} \otimes v_{2} \otimes v_{3} \otimes \otimes v_{4}\right)=\tau_{\text {medial }} \circ \rho\left(M^{(4)} \mid\left(v_{1} \otimes v_{2} \otimes v_{3} \otimes v_{4}\right)\right) \\
& =\tau_{\text {medial }} \circ\left(\sum_{\alpha} \rho\left(\mathrm{m}_{\alpha}^{(1)} \mid v_{1}\right) \otimes \rho\left(\mathrm{m}_{\alpha}^{(2)} \mid v_{2}\right) \otimes \rho\left(\mathrm{m}_{\alpha}^{(3)} \mid v_{3}\right) \otimes \rho\left(\mathrm{m}_{\alpha}^{(4)} \mid v_{4}\right)\right), \\
& v_{i} \in V_{i}, \quad \mathrm{~m}_{\alpha}^{(i)} \in B, \quad i=1,2,3,4
\end{aligned}
$$


where $\tau_{\text {medial }}$ is the medial map (286), and $\rho: B \times V \rightarrow V$ is the ordinary 1-place action (7).

After inserting (298) into (293) and (294), using (297) we obtain the equations for $M$-matrix

$$
\begin{aligned}
& \mathscr{M}_{1546}^{(7)} \mathscr{M}_{5643}^{(7)} \mathscr{M}_{6342}^{(7)} \mathscr{M}_{3247}^{(7)} \mathscr{M}_{1653}^{(7)} \mathscr{M}_{6352}^{(7)} \mathscr{M}_{3254}^{(7)} \mathscr{M}_{2457}^{(7)} \mathscr{M}_{1362}^{(7)} \mathscr{M}_{3264}^{(7)} \mathscr{M}_{2465}^{(7)} \mathscr{M}_{4567}^{(7)} \mathscr{M}_{1234}^{(7)} \\
& =\mathscr{M}_{6237}^{(7)} \mathscr{M}_{1546}^{(7)} \mathscr{M}_{5642}^{(7)} \mathscr{M}_{6243}^{(7)} \mathscr{M}_{2347}^{(7)} \cdot \mathscr{M}_{1652}^{(7)} \cdot \mathscr{M}_{6253}^{(7)} \cdot \mathcal{M}_{2354}^{(7)} \cdot \mathcal{M}_{3457}^{(7)} \mathscr{M}_{1263}^{(7)} \mathscr{M}_{2364}^{(7)} \mathscr{M}_{3465}^{(7)} \mathscr{M}_{4567}^{(7)}
\end{aligned}
$$

and

$$
\begin{aligned}
& \mathscr{M}_{5243}^{(7)} \mathscr{M}_{2347}^{(7)} \mathscr{M}_{1562}^{(7)} \mathscr{M}_{5263}^{(7)} \mathscr{M}_{2364}^{(7)} \mathscr{M}_{3467}^{(7)} \mathscr{M}_{1253}^{(7)} \mathscr{M}_{2354}^{(7)} \mathscr{M}_{3456}^{(7)} \\
& =\mathscr{M}_{6452}^{(7)} \mathscr{M}_{4253}^{(7)} \mathscr{M}_{2357}^{(7)} \mathscr{M}_{1462}^{(7)} \mathscr{M}_{4263}^{(7)} \mathscr{M}_{2365}^{(7)} \mathscr{M}_{3567}^{(7)} \mathscr{M}_{1243}^{(7)} \mathscr{M}_{2345}^{(7)}
\end{aligned}
$$

which respect the braid equations (293) and (294).

Remark 151. The unequal number of terms in (299) and (300) is governed by different commutative diagrams of modules (293) and (294), respectively (cf. (247) and (250)-(252)).

\section{Medial analog of triangularity}

Now we consider the possible analogs of the quasitriangularity conditions (similar to (245)-(246) and quasipolyangularity $(273-(275))$ for a polyadic almost co-medial bialgebra $B^{\left(n^{\prime}, n\right)}$.

Definition 152. A polyadic almost co-medial bialgebra $B^{\left(n^{\prime}, n\right)}=\left\langle B \mid \mu^{(n)}, \Delta^{\left(n^{\prime}\right)}\right\rangle$ with the polyadic $M$ matrix $M^{\left(n^{\prime 2}\right)}=\sum_{\alpha} \mathrm{m}_{\alpha}^{(1)} \otimes \ldots \otimes \mathrm{m}_{\alpha}^{\left(n^{\prime 2}\right)}, \quad \mathrm{m}_{\alpha}^{(i)} \in B$ is called medial quasipolyangular, if the following $n^{\prime 2}$ relations hold

$$
\begin{aligned}
& \sum_{\alpha}\left(\Delta^{\left(n^{\prime}\right)}\right)^{\otimes n^{\prime}} \circ \Delta^{\left(n^{\prime}\right)}\left(\mathrm{m}_{\alpha}^{(1)}\right) \otimes \mathrm{m}_{\alpha}^{(2)} \otimes \ldots \otimes \mathrm{m}_{\alpha}^{\left(n^{\prime 2}\right)}=\sum_{\alpha_{1}, \ldots \alpha_{n^{\prime}}} \mathrm{m}_{\alpha_{1}}^{(1)} \otimes \mathrm{m}_{\alpha_{2}}^{(1)} \otimes \ldots \otimes \mathrm{m}_{\alpha_{n^{\prime 2}}}^{(1)} \\
& \otimes\left(\mu^{(n)}\right)^{\circ \ell}\left[\mathrm{m}_{\alpha_{1}}^{(2)} \otimes \mathrm{m}_{\alpha_{2}}^{(2)} \otimes \ldots \otimes \mathrm{m}_{\alpha_{n^{\prime}}}^{(2)}\right] \otimes \ldots \otimes\left(\mu^{(n)}\right)^{\propto \ell}\left[\mathrm{m}_{\alpha_{1}}^{\left(n^{\prime 2}\right)} \otimes \mathrm{m}_{\alpha_{2}}^{\left(n^{\prime 2}\right)} \otimes \ldots \otimes \mathrm{m}_{\alpha_{n^{\prime 2}}}^{\left(n^{\prime 2}\right)}\right], \\
& \sum_{\alpha} \mathrm{m}_{\alpha}^{(1)} \otimes\left(\Delta^{\left(n^{\prime}\right)}\right)^{\otimes n^{\prime}} \circ \Delta^{\left(n^{\prime}\right)}\left(\mathrm{m}_{\alpha}^{(2)}\right) \otimes \ldots \otimes \mathrm{m}_{\alpha}^{\left(n^{\prime 2}\right)} \\
& =\sum_{\alpha_{1}, \ldots, \alpha_{n^{\prime}}}\left(\mu^{(n)}\right)^{\circ \ell} \circ \tau_{\text {medial }}^{\left(n^{\prime 2}, n^{\prime 2}\right)}\left[\mathrm{m}_{\alpha_{1}}^{(1)} \otimes \mathrm{m}_{\alpha_{2}}^{(1)} \otimes \ldots \otimes \mathrm{m}_{\alpha_{n^{\prime 2}}}^{(1)}\right] \otimes \mathrm{m}_{\alpha_{1}}^{(2)} \otimes \mathrm{m}_{\alpha_{2}}^{(2)} \otimes \ldots \otimes \mathrm{m}_{\alpha_{n^{\prime 2}}}^{(2)} \otimes \ldots \\
& \otimes\left(\mu^{(n)}\right)^{\alpha \ell}\left[\mathrm{m}_{\alpha_{1}}^{\left(n^{\prime 2}\right)} \otimes \mathrm{m}_{\alpha_{2}}^{\left(n^{\prime 2}\right)} \otimes \ldots \otimes \mathrm{m}_{\alpha_{n^{\prime 2}}}^{\left(n^{\prime 2}\right)}\right] \\
& \sum_{\alpha} \mathrm{m}_{\alpha}^{(1)} \otimes \ldots \otimes \mathrm{m}_{\alpha}^{\left(n^{\prime 2}-1\right)} \otimes\left(\Delta^{\left(n^{\prime}\right)}\left(\mathrm{m}_{\alpha}^{\left(n^{\prime 2}\right)}\right)\right)^{\otimes n^{\prime}} \circ \Delta^{\left(n^{\prime}\right)}\left(\mathrm{m}_{\alpha}^{\left(n^{\prime 2}\right)}\right) \\
& =\sum_{\alpha_{1}, \ldots, \alpha_{n^{\prime 2}}}\left(\mu^{(n)}\right)^{\circ \ell} \circ \tau_{\text {medial }}^{\left(n^{\prime 2}, n^{\prime 2}\right)}\left[\mathrm{m}_{\alpha_{1}}^{(1)} \otimes \mathrm{m}_{\alpha_{2}}^{(1)} \otimes \ldots \otimes \mathrm{m}_{\alpha_{n^{\prime}}}^{(1)}\right] \otimes \ldots \\
& \otimes\left(\mu^{(n)}\right)^{\circ \ell} \circ \tau_{\text {medial }}^{\left(n^{\prime 2}, n^{\prime 2}\right)}\left[\mathrm{m}_{\alpha_{1}}^{\left(n^{\prime 2}-1\right)} \otimes \mathrm{m}_{\alpha_{2}}^{\left(n^{\prime 2}-1\right)} \otimes \ldots \otimes \mathrm{m}_{\alpha_{n^{\prime 2}}}^{\left(n^{\prime 2}-1\right)}\right] \otimes \mathrm{m}_{\alpha_{1}}^{\left(n^{\prime 2}\right)} \otimes \mathrm{m}_{\alpha_{2}}^{\left(n^{\prime 2}\right)} \otimes \ldots \otimes \mathrm{m}_{\alpha_{n^{\prime}}}^{\left(n^{\prime 2}\right)},
\end{aligned}
$$

where $\tau_{\text {medial }}^{\left(n^{\prime 2}, n^{\prime 2}\right)}$ is the unique medial twist map (69). The arity shape of a medial quasipolyangular bialgebra $B^{\left(n^{\prime}, n\right)}$ is given by (cf. (276))

$$
n^{\prime 2}=\ell(n-1)+1, \quad \ell \in \mathbb{N} .
$$

Remark 153. Similar to Remark 138, the medial quasipolyangularity equations (301)-(303) can be expressed in terms of the extended $M$-matrix for the first equation (301) and the last one (303) only, because in the intermediate equations the sequences of $M$-matrix elements are permuted. 
Example 154. In the case where $n^{\prime}=n=2, \ell=3$, for the bialgebra $B^{(2,2)}=\langle B \mid \mu=(\cdot), \Delta\rangle$ with the polyadic $M$-matrix

$$
M^{(4)}=\sum_{\alpha} \mathrm{m}_{\alpha}^{(1)} \otimes \mathrm{m}_{\alpha}^{(2)} \otimes \mathrm{m}_{\alpha}^{(3)} \otimes \mathrm{m}_{\alpha}^{(4)}, \quad \mathrm{m}_{\alpha}^{(i)} \in B
$$

we have the binary medial quasipolyangularity equations

$$
\begin{aligned}
& \sum_{\alpha}(\Delta \otimes \Delta) \circ \Delta\left(\mathrm{m}_{\alpha}^{(1)}\right) \otimes \mathrm{m}_{\alpha}^{(2)} \otimes \mathrm{m}_{\alpha}^{(3)} \otimes \mathrm{m}_{\alpha}^{(4)}=\sum_{\alpha_{1}, \alpha_{2}, \alpha_{3}, \alpha_{4}} \mathrm{~m}_{\alpha_{1}}^{(1)} \otimes \mathrm{m}_{\alpha_{2}}^{(1)} \otimes \mathrm{m}_{\alpha_{3}}^{(1)} \otimes \mathrm{m}_{\alpha_{4}}^{(1)} \\
& \otimes \mathrm{m}_{\alpha_{1}}^{(2)} \cdot \mathrm{m}_{\alpha_{2}}^{(2)} \cdot \mathrm{m}_{\alpha_{3}}^{(2)} \cdot \mathrm{m}_{\alpha_{4}}^{(2)} \otimes \mathrm{m}_{\alpha_{1}}^{(3)} \cdot \mathrm{m}_{\alpha_{2}}^{(3)} \cdot \mathrm{m}_{\alpha_{3}}^{(3)} \cdot \mathrm{m}_{\alpha_{4}}^{(3)} \otimes \mathrm{m}_{\alpha_{1}}^{(4)} \cdot \mathrm{m}_{\alpha_{2}}^{(4)} \cdot \mathrm{m}_{\alpha_{3}}^{(4)} \cdot \mathrm{m}_{\alpha_{4}}^{(4)}, \\
& \sum_{\alpha} \mathrm{m}_{\alpha}^{(1)} \otimes(\Delta \otimes \Delta) \circ \Delta\left(\mathrm{m}_{\alpha}^{(2)}\right) \otimes \mathrm{m}_{\alpha}^{(3)} \otimes \mathrm{m}_{\alpha}^{(4)}=\sum_{\alpha_{1}, \alpha_{2}, \alpha_{3}, \alpha_{4}} \mathrm{~m}_{\alpha_{1}}^{(1)} \cdot \mathrm{m}_{\alpha_{3}}^{(1)} \cdot \mathrm{m}_{\alpha_{2}}^{(1)} \cdot \mathrm{m}_{\alpha_{4}}^{(1)} \\
& \otimes \mathrm{m}_{\alpha_{1}}^{(2)} \otimes \mathrm{m}_{\alpha_{2}}^{(2)} \otimes \mathrm{m}_{\alpha_{3}}^{(2)} \otimes \mathrm{m}_{\alpha_{4}}^{(2)} \otimes \mathrm{m}_{\alpha_{1}}^{(3)} \cdot \mathrm{m}_{\alpha_{2}}^{(3)} \cdot \mathrm{m}_{\alpha_{3}}^{(3)} \cdot \mathrm{m}_{\alpha_{4}}^{(3)} \otimes \mathrm{m}_{\alpha_{1}}^{(4)} \cdot \mathrm{m}_{\alpha_{2}}^{(4)} \cdot \mathrm{m}_{\alpha_{3}}^{(4)} \cdot \mathrm{m}_{\alpha_{4}}^{(4)}, \\
& \sum_{\alpha} \mathrm{m}_{\alpha}^{(1)} \otimes \mathrm{m}_{\alpha}^{(2)} \otimes(\Delta \otimes \Delta) \circ \Delta\left(\mathrm{m}_{\alpha}^{(3)}\right) \otimes \mathrm{m}_{\alpha}^{(4)}=\sum_{\alpha_{1}, \alpha_{2}, \alpha_{3}, \alpha_{4}} \mathrm{~m}_{\alpha_{1}}^{(1)} \cdot \mathrm{m}_{\alpha_{3}}^{(1)} \cdot \mathrm{m}_{\alpha_{2}}^{(1)} \cdot \mathrm{m}_{\alpha_{4}}^{(1)} \\
& \otimes \mathrm{m}_{\alpha_{1}}^{(2)} \cdot \mathrm{m}_{\alpha_{3}}^{(2)} \cdot \mathrm{m}_{\alpha_{2}}^{(2)} \cdot \mathrm{m}_{\alpha_{4}}^{(2)} \otimes \mathrm{m}_{\alpha_{1}}^{(3)} \otimes \mathrm{m}_{\alpha_{2}}^{(3)} \otimes \mathrm{m}_{\alpha_{3}}^{(3)} \otimes \mathrm{m}_{\alpha_{4}}^{(3)} \otimes \mathrm{m}_{\alpha_{1}}^{(4)} \cdot \mathrm{m}_{\alpha_{2}}^{(4)} \cdot \mathrm{m}_{\alpha_{3}}^{(4)} \cdot \mathrm{m}_{\alpha_{4}}^{(4)}, \\
& \sum_{\alpha} \mathrm{m}_{\alpha}^{(1)} \otimes \mathrm{m}_{\alpha}^{(2)} \otimes \mathrm{m}_{\alpha}^{(3)} \otimes(\Delta \otimes \Delta) \circ \Delta\left(\mathrm{m}_{\alpha}^{(4)}\right)=\sum_{\alpha_{1}, \alpha_{2}, \alpha_{3}, \alpha_{4}} \mathrm{~m}_{\alpha_{1}}^{(1)} \cdot \mathrm{m}_{\alpha_{3}}^{(1)} \cdot \mathrm{m}_{\alpha_{2}}^{(1)} \cdot \mathrm{m}_{\alpha_{4}}^{(1)} \\
& \otimes \mathrm{m}_{\alpha_{1}}^{(2)} \cdot \mathrm{m}_{\alpha_{3}}^{(2)} \cdot \mathrm{m}_{\alpha_{2}}^{(2)} \cdot \mathrm{m}_{\alpha_{4}}^{(2)} \otimes \mathrm{m}_{\alpha_{1}}^{(3)} \cdot \mathrm{m}_{\alpha_{3}}^{(3)} \cdot \mathrm{m}_{\alpha_{2}}^{(3)} \cdot \mathrm{m}_{\alpha_{4}}^{(3)} \otimes \mathrm{m}_{\alpha_{1}}^{(4)} \otimes \mathrm{m}_{\alpha_{2}}^{(4)} \otimes \mathrm{m}_{\alpha_{3}}^{(4)} \otimes \mathrm{m}_{\alpha_{4}}^{(4)} .
\end{aligned}
$$

According to Remark 153, we can express through the extended $M$-matrix (297) the first medial quasipolyangularity equation (306) and the last one (309) only, as follows

$$
\begin{aligned}
& \left((\Delta \otimes \Delta) \circ \Delta \otimes \operatorname{id}_{B} \otimes \operatorname{id}_{B} \otimes \operatorname{id}_{B}\right)\left(M^{(4)}\right)=\mathcal{M}_{1567}^{(7)} \cdot \mathcal{M}_{2567}^{(7)} \cdot \mathcal{M}_{3567}^{(7)} \cdot \mathcal{M}_{4567}^{(7)}, \\
& \left(\operatorname{id}_{B} \otimes \operatorname{id}_{B} \otimes \operatorname{id}_{B} \otimes(\Delta \otimes \Delta) \circ \Delta\right)\left(M^{(4)}\right)=\mathcal{M}_{1234}^{(7)} \cdot \mathcal{M}_{1236}^{(7)} \cdot \mathcal{M}_{1235}^{(7)} \cdot \mathcal{M}_{1237}^{(7)} .
\end{aligned}
$$

The compatibility of (310)-(311) with the (binary) almost co-mediality (281) leads to

Proposition 155. An extended binary $M$-matrix (297) of the binary almost co-medial bialgebra $B^{(2,2)}=$ $\langle B \mid \mu=(\cdot), \Delta\rangle$ satisfies the compatibility equations (cf. (247))

$$
\begin{aligned}
& \mathscr{M}_{1234}^{(7)} \cdot \mathscr{M}_{1567}^{(7)} \cdot \mathscr{M}_{2567}^{(7)} \cdot \mathscr{M}_{3567}^{(7)} \cdot \mathscr{M}_{4567}^{(7)}=\mathscr{M}_{1567}^{(7)} \cdot \mathscr{M}_{3567}^{(7)} \cdot \mathscr{M}_{2567}^{(7)} \cdot \mathscr{M}_{4567}^{(7)} \cdot \mathscr{M}_{1234}^{(7)}, \\
& \mathscr{M}_{4567}^{(7)} \cdot \mathscr{M}_{1234}^{(7)} \cdot \mathscr{M}_{1236}^{(7)} \cdot \mathscr{M}_{1235}^{(7)} \cdot \mathscr{M}_{1237}^{(7)}=\mathscr{M}_{1234}^{(7)} \cdot \mathscr{M}_{1235}^{(7)} \cdot \mathscr{M}_{1236}^{(7)} \cdot \mathscr{M}_{1237}^{(7)} \cdot \mathscr{M}_{4567}^{(7)} .
\end{aligned}
$$

Proof. The identities for the $M$-matrix

$$
\begin{aligned}
& \left(M^{(4)} \otimes \operatorname{id}_{B} \otimes \operatorname{id}_{B} \otimes \operatorname{id}_{B}\right) \circ\left((\Delta \otimes \Delta) \circ \Delta \otimes \operatorname{id}_{B} \otimes \operatorname{id}_{B} \otimes \operatorname{id}_{B}\right)\left(M^{(4)}\right) \\
& =\left(\tau_{\text {medial }} \circ(\Delta \otimes \Delta) \circ \Delta \otimes \operatorname{id}_{B} \otimes \operatorname{id}_{B} \otimes \operatorname{id}_{B}\right)\left(M^{(4)}\right) \circ\left(M^{(4)} \otimes \operatorname{id}_{B} \otimes \operatorname{id}_{B} \otimes \operatorname{id}_{B}\right), \\
& \left(\operatorname{id}_{B} \otimes \operatorname{id}_{B} \otimes \operatorname{id}_{B} \otimes M^{(4)}\right) \circ\left(\operatorname{id}_{B} \otimes \operatorname{id}_{B} \otimes \operatorname{id}_{B} \otimes(\Delta \otimes \Delta) \circ \Delta\right)\left(M^{(4)}\right) \\
& =\left(\operatorname{id}_{B} \otimes \operatorname{id}_{B} \otimes \operatorname{id}_{B} \otimes \tau_{\text {medial }} \circ(\Delta \otimes \Delta) \circ \Delta\right)\left(M^{(4)}\right) \circ\left(\operatorname{id}_{B} \otimes \operatorname{id}_{B} \otimes \operatorname{id}_{B} \otimes M^{(4)}\right),
\end{aligned}
$$

follow from the almost co-mediality condition(281), and then we apply quasipolyangularity (310)-(311).

Remark 156. Two other compatibility equations corresponding to the intermediate quasipolyangularity equations (307)-(308) can be written in component form only.

The solutions to (312)-(313) can be found in matrix form by choosing an appropriate basis and using the standard methods (see, e.g., $[7,49])$. 


\section{CONCLUSIONS}

We have presented the "polyadization" procedure of the following algebra-like structures: algebras, coalgebras, bialgebras and Hopf algebras (see [10,20] for ring-like structures). In our concrete constructions the initial arities of operations are taken as arbitrary, and we then try to restrict them only by means of natural relations which bring to mind the binary case. This leads to many exotic properties and unexpected connections between arities and a fixing of their values called "quantization". For instance, the unit and counit (which do not always exist) can be multivalued many place maps, polyadic algebras can be zeroless, the qeurelements should be considered instead of inverse elements under addition and multiplication, a polyadic bialgebra can consist of an algebra and coalgebra of different arities, and a polyadic analog of Hopf algebras contains (instead of the ordinary antipode) the querantipode, which has different properties.

The formulas and constructions introduced for concrete algebra-like structures can have many applications, e.g., in combinatorics, quantum logic, or representation theory. As an example, we have introduced possible polyadic analogs of braidings, almost co-commutativity and a version of the R-matrix. A new concept of deformation (using the medial map) is proposed: this is unique and therefore can be more consequential and suitable in the polyadic case.

(D)Steven Duplij, https://orcid.org/0000-0003-1184-6952

\section{ORCID IDs}

\section{REFERENCES}

[1] M.E. Sweedler, Hopf Algebras. (W.A. Benjamin, New York, 1969).

[2] E. Abe, Hopf Algebras, (Cambridge Univ. Press, Cambridge, 1980).

[3] D.E. Radford, Hopf Algebras, (World Scientific, Hackensack, 2012).

[4] V.G. Drinfeld, in Proceedings of the ICM, Berkeley, edited by A. Gleason (AMS, Phode Island, 1987), pp. 798.

[5] S. Shnider, and S. Sternberg, Quantum Groups, (International Press, Boston, 1993).

[6] V. Chari, and A. Pressley, A Guide to Quantum Groups, (Cambridge University Press, Cambridge, 1996).

[7] C. Kassel, Quantum Groups, (Springer-Verlag, New York, 1995).

[8] S. Majid, Foundations of Quantum Group Theory, (Cambridge University Press, Cambridge, 1995).

[9] G. Karaali, Commun. Algebra, 36, 4341 (2008), https://doi.org/10.1080/00927870802182424.

[10] S. Duplij, J. Math. Physics, Analysis, Geometry, 15(1), 3-56 (2019), (to appear), https://arxiv.org/abs/1703.10132.

[11] J.A. de Azcarraga, and J. M. Izquierdo, J. Phys. A, 43, 293001 (2010), https://doi.org/10.1088/1751-8113/43/29/293001.

[12] P.W. Michor, and A.M. Vinogradov, Rend. Sem. Mat. Univ. Pol. Torino, 54, 373 (1996), http://www.seminariomatematico.polito.it/rendiconti/cartaceo/54-4/373.pdf.

[13] M. Goze, and M. Rausch de Traubenberg, J. Math. Phys. 50, 063508 (2009), https://doi.org/10.1063/1.3152631.

[14] S. Duplij, in: Exotic Algebraic and Geometric Structures in Theoretical Physics, edited by S. Duplij (Nova Publishers, New York, 2018), pp. 251, arXiv: math.RT/1308.4060, https://arxiv.org/abs/1308.4060.

[15] M. Mark1, S. Shnider, and J. Stasheff, Operads in Algebra, Topology and Physics, (AMS, Providence, 2002).

[16] J.-L. Loday, and B. Vallette, Algebraic operads, (Springer, Heidelberg, 2012).

[17] W. Dörnte, Mathematische Zeitschrift, 29, 1-19 (1929), https://doi.org/10.1007/BF01180515.

[18] G. Crombez, Abh. Math. Semin. Univ. Hamb. 37, 180 (1972), https://doi.org/10.1007/BF02999695.

[19] J.J. Leeson, and A.T. Butson, Algebra Univers. 11, 42 (1980), https://doi.org/10.1007/BF02483082.

[20] S. Duplij, p-Adic Numbers, Ultrametric Analysis and Appl. 9, 267-291 (2017), https://arxiv.org/abs/1707.00719.

[21] J. Ušan, Mathematica Moravica, Special vol., $203 \quad$ (2003), http://www.moravica.ftn.kg.ac.rs/Special/nGroups_in_the_Light_of_Natural_Operations-v2006.pdf.

[22] S. Duplij, and W. Werner, Structure of unital 3-fields, (2015), https://arxiv.org/abs/1505.04393v1.

[23] Takeo Yokonuma, Tensor Spaces and Exterior Algebra, (AMS, Providence, 1992).

[24] S. Duplij, in: Symmetry in Nonlinear Mathematical Physics, edited by A.G. Nikitin, and V.M. Boyko (Institute of Mathematics, Kiev, 2001), pp. 25.

[25] S. Duplij, in: Exotic Algebraic and Geometric Structures in Theoretical Physics, edited by S. Duplij (Nova Publishers, New York, 2018), pp. 309.

[26] T. Evans, Duke Math J. 30, 331 (1963), https://doi.org/10.1215/S0012-7094-63-03035-7.

[27] V.D. Belousov, n-Ary Quasigroups, (Shtintsa, Kishinev, 1972).

[28] C.W. Curtis, and I. Reiner, Representation theory of finite groups and associative algebras, (AMS, Providence, 1962).

[29] A.A. Kirillov, Elements of the Theory of Representations, (Springer-Verlag, Berlin, 1976).

[30] M. Hazewinkel, N. Gubareni, and V.V. Kirichenko, Algebras, Rings and Modules: Lie Algebras and Hopf Algebras, (AMS, Providence, 2010).

[31] A. Borowiec, W. Dudek, and S. Duplij, Commun. Algebra, 34, 1651 (2006), https://doi.org/10.1080/00927870500542564.

[32] M.E. Sweedler, J. Algebra, 8, 262 (1968), https://doi.org/10.1016/0021-8693(68)90059-8.

[33] R.G. Heyneman, and M.E. Sweedler, J. Algebra, 13, 192 (1969), https://doi.org/10.1016/0021-8693(69)90071-4.

[34] Y. Kashina, S. Montgomery, and S.-H. Ng, Israel J. Math. 188, 57 (2012), https://doi.org/10.1007/s11856-011-0092-7.

[35] M. Aguiar, and A. Lauve, Algebra Number Theory, 9, 547 (2015), https://doi.org/10.2140/ant.2015.9.547.

[36] E.L. Post, Trans. Amer. Math. Soc. 48, 208 (1940), https://doi.org/10.2307/1990085. 
[37] W.A. Dudek, Discuss. Math., Gen. Algebra Appl. 27, 199 (2007), http://dx.doi.org/10.7151/dmgaa.

[38] E. Heine, Handbuch der Kugelfunktionan, (Reimer, Berlin, 1878).

[39] V. Kac, and P. Cheung, Quantum calculus, (Springer, New York, 2002).

[40] S. Duplij, Pure Math. Appl. 9, 283 (1998), http://duplij.univer.kharkov.ua/puma.pdf.gz.

[41] S. Duplij and W. Marcinek, Nucl. Phys. Proc. Suppl. 102, 293 (2001), Int. Conference on Supersymmetry and Quantum Field Theory: D.V. Volkov Memorial Conference, (Kharkov, Ukraine, 2000).

[42] S. Duplij, and W. Marcinek, J. Math. Phys. 43, 3329 (2002), https://doi.org/10.1063/1.1473681.

[43] S. Duplij, and W. Marcinek, in: Exotic Algebraic and Geometric Structures in Theoretical Physics, edited by S. Duplij, (Nova Publishers, New York, 2018), pp. 15.

[44] S. Duplij, and F. Li, Czech. J. Phys. 51, 1306 (2001), https://doi.org/10.1023/A:1013313802053.

[45] F. Li, and S. Duplij, Commun. Math. Phys. 225, 191 (2002), https://doi.org/10.1007/s002201000576.

[46] K. Szlach'anyi, in: Operator Algebras and Quantum Field Theory, edited by S. Doplicher, R. Longo, J.E. Roberts, and L. Zsid'o (International Press, New York, 1996), p. 221.

[47] A.M. Gal'mak, n-Ary Groups, Part 1, (Gomel University, Gomel, 2003).

[48] V.G. Drinfeld, Leningrad Math. J. 1, 321 (1989), http://www.mathnet.ru/php/getFT.phtml?jrnid=aa\&paperid=10\&what= fullt\&option_lang=rus. (in Russian)

[49] L.A. Lambe, and D.E. Radford, Introduction to the Quantum Yang-Baxter Equation and Quantum Groups: An Algebraic Approach, (Kluwer, Dordrecht, 1997).

[50] V.G. Drinfeld, in: Problems of Modern Quantum Field Theory, edited by A.A. Belavin, A.V. Klimyk, and A.B. Zamolodchikov (Springer-Verlag, Heidelberg, 1989), pp. 1.

\section{ПОЛІАДИЧНІ АЛГЕБРИ ХОПФА І КВАНТОВІ ГРУПИ \\ С.А. Дуплій}

Центр інформатики, Університет Монстеру, Німеччина

Ця стаття продовжує вивчення конкретних алгеброподібних структур у нашому поліадичному підході, де арності всіх операцій спочатку приймаються як довільні, але відносини між ними, форми арності, повинні бути знайдені 3 деяких природних умов («принцип свободи арності»). Таким чином, визначаються і досліджуються узагальнені асоціативні алгебри, коасоціативні коалгебри, біалгебри і алгебри Хопфа. Вони мають багато незвичайних особливостей у порівнянні 3 бінарним випадком. Наприклад, і алгебра, і поле, що лежить в її основі можуть бути нульовими і непоодинокими, існування одиниці і лічильника не обов'язково, а розмірність алгебри не довільна, а «квантована». Можуть бути визначені поліадичний твір згортки і біалгебра, і коли алгебра і коалгебра мають нерівні аріти, поліадична версія антипода, querantipode, має різні властивості. Як можливе застосування до квантової теорії груп ми вводимо поліадичну версію плетінь, майже кокоммутативність, квазітриангулярність і рівняння для R-матриці (які можна розглядати як поліадичний аналог рівняння Янга-Бакстера). Ми пропонуємо іншу концепцію деформації, яка керується не картою твіст, а медіальною картою, де тільки остання унікальна в поліадичному випадку.

КЛЮЧОВІ СЛОВА: поліадичне поле, поліадична алгебра, біалгебра, алгебра Хопфа, антипод, рівняння коси, плетіння, R-матриця, рівняння Янга-Бакстера, медіальність, ко-медіальність, М-матриця, квазітриангулярність 